\title{
World Agricultural Production
}

Circular Series

WAP 4-23

April 2023

\section{Argentina Corn: Production Down 25 Percent from Last Year}

Argentina corn production for marketing year 2022/23 is estimated at 37.0 million metric tons, down 8 percent from last month, and down 25 percent from last year. Corn yield is estimated at 5.52 tons per hectare, down 7 percent from last month, and down 21 percent from last year. Harvested area is estimated at 6.7 million hectares, unchanged from last month, and down 6 percent from last year.

Argentina Maximum Daily Temperature (CPC)

Highest Value from March 1 to March 31, 2023

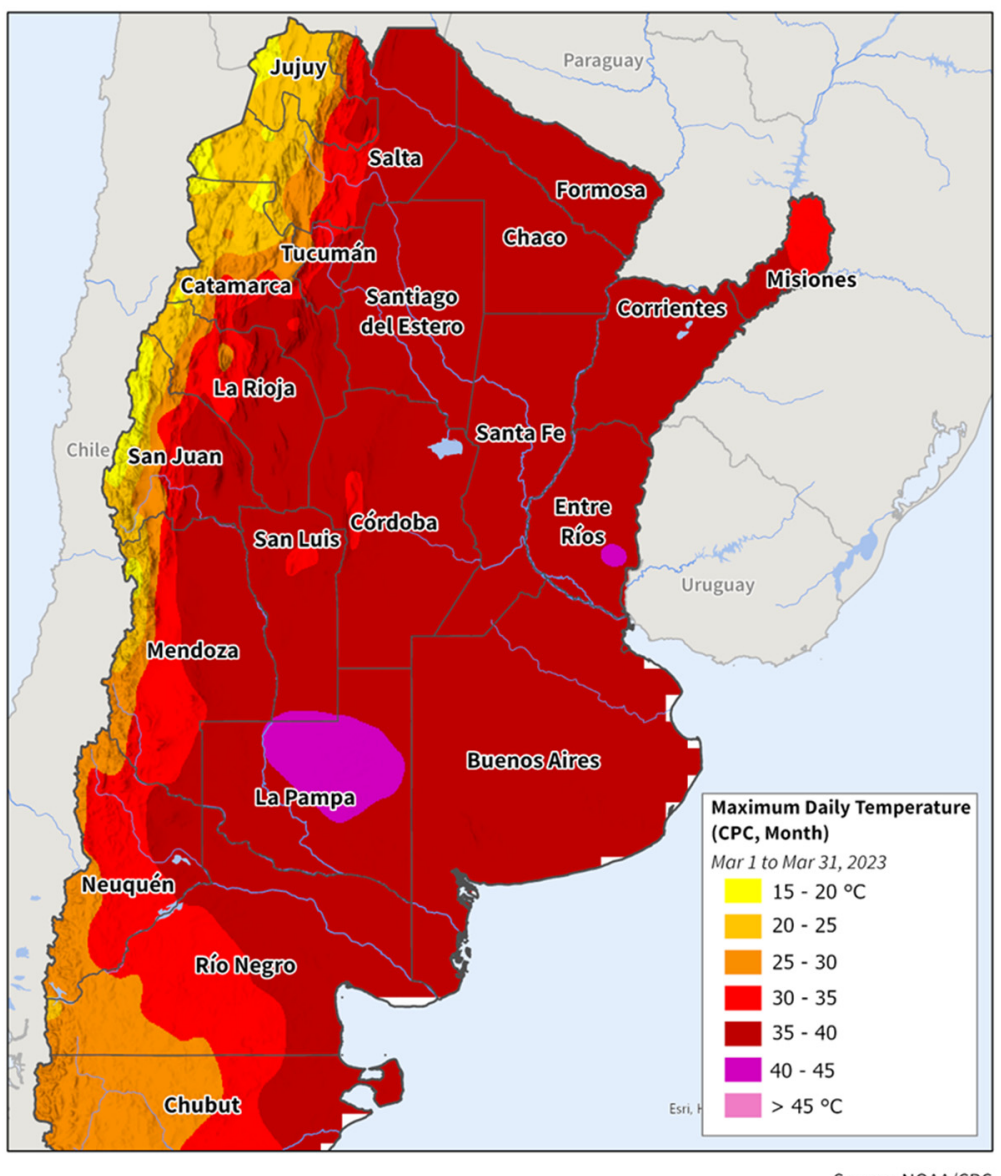

Source: NOAA/CPC 
Yield is down month-to-month due to heat and dryness that impacted the late-planted corn, which accounts for roughly two-thirds of area. Temperatures during March were well above normal, with many days above 35 degrees Celsius. Dryness continued until about late March when rainfall occurred. According to FAS Buenos Aires, who conducted crop assessment during March, the late-March rainfall was too late to benefit the early-planted corn and may only marginally help the late-planted corn. Harvest for corn has begun and according to the Bolsa Cereales in Buenos Aires, about 10 percent of the crop has been harvested as of early April. Yields are well-below average. Harvest will continue until early August. (For more information, please contact Katie.McGaughey@usda.gov.)

\section{Brazil Corn: No Change to Production for MY22/23 as Planting Concludes}

Brazil corn production for marketing year (MY) 2022/23 is estimated at a record 125.0 million metric tons (mmt), unchanged from last month, and larger than last year's crop by $9.0 \mathrm{mmt}(8$ percent). Total harvested area, for all three corn crops, is estimated at a record 22.7 million hectares (mha), up 0.9 mha (4 percent) from last year. Yield is estimated at 5.51 tons per hectare, 3 percent above last year's crop, and 6 percent above the 5-year average yield.

The first-season crop is in the middle of harvest at 48 percent completed as of April 3 per the Companhia Nacional de Abastecimento (CONAB). Harvested area for this crop is estimated at 4.4 mha, with production at $26.8 \mathrm{mmt}$ according to CONAB - roughly $2.0 \mathrm{mmt}$ lower than initial forecasts. Yield expectations are mixed with several states in the Northeast and the state of Rio Grande do Sul experiencing dry conditions at critical pollination and grain-filling stages. These areas will display lower yields, whereas other states (for example, Paraná and Santa Catarina) are expecting exceptional yields.

\section{Brazil Corn: 2-Month Percent of Normal Precipitation and NDVI}

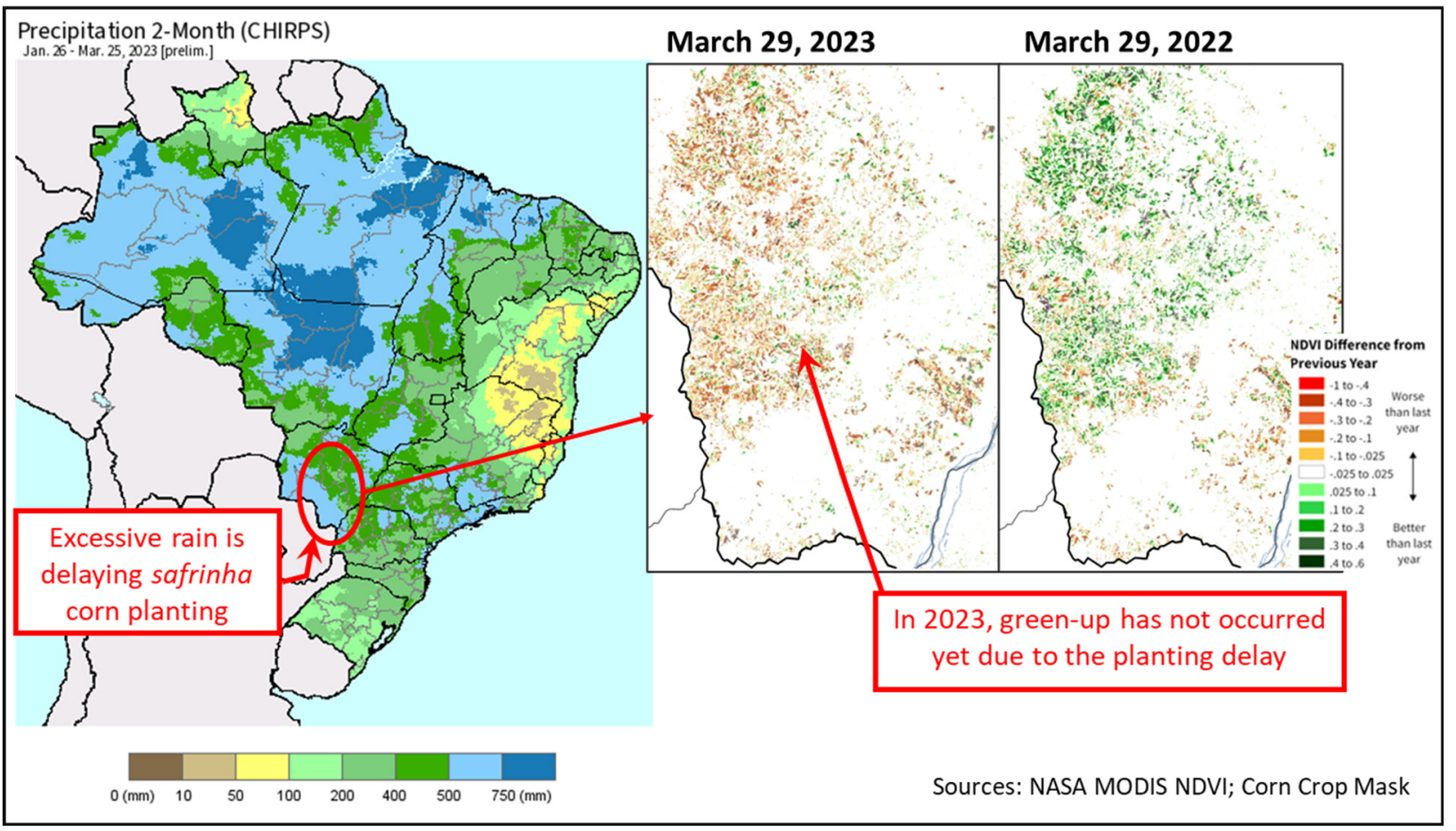


Planting for the second-season crop is mostly complete except in the state of Mato Grosso do Sul (12 percent of safrinha production) where excessive soil moisture has delayed planting by 24 percent relative to the 5-year average. In the largest safrinha state of Mato Grosso (47 percent of safrinha production), farmers planted about 80 percent of the crop within the ideal sowing window on a record area, according to the Instituto Mato-Grossense de Economia Agropecuária (IMEA). Some producers who did not sow within the ideal window are opting for other crops, such as wheat in Paraná, as a late safrinha planting raises the risk of lower yields. The second-season corn area is estimated by CONAB at a record $17.0 \mathrm{mha}$, up $0.6 \mathrm{mha}$ from last year. Sufficient rainfall and intermittent sunshine have been beneficial for crop development so far this season. The second corn crop is harvested from June to September. (For more information, please contact Sunita.Yadav-Pauletti@usda.gov.)

\section{Russia Corn: High Production Despite Delayed Harvest}

\section{Evaporative Stress Index (12-weeks ending August 6, 2022)}

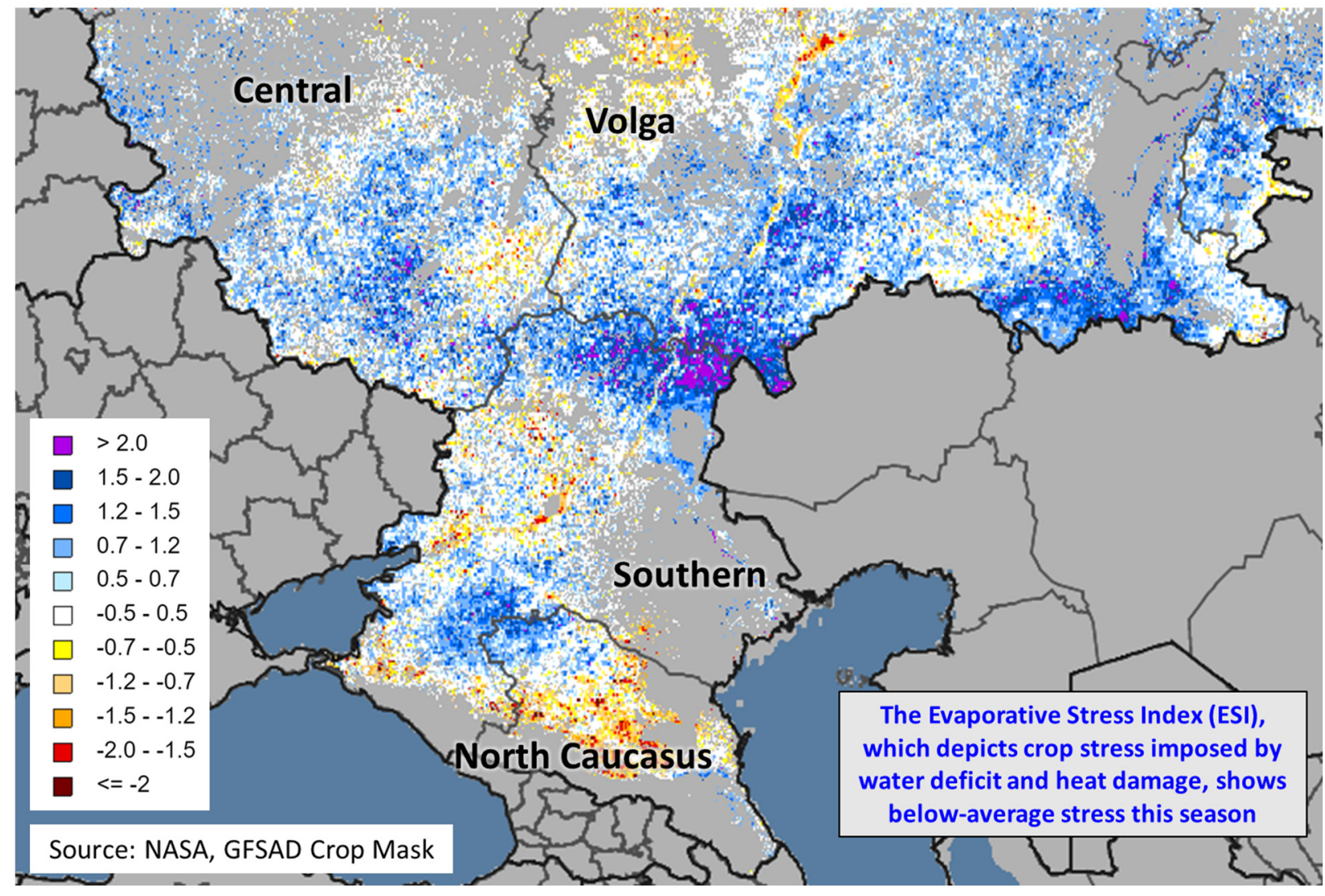

Russia corn production for marketing year 2022/23 is estimated at 15.8 million metric tons (mmt), up 13 percent from last month and 4 percent from last year. Total corn yield is estimated at a record 6.00 tons per hectare $(\mathrm{t} / \mathrm{ha})$, up 7 percent from last month and 14 percent from last year. Total harvested area is estimated at 2.6 million hectares (mha), up 6 percent from last month, but down 9 percent from last year. 


\section{Percent Normal Rainfall for the Month of July 2022}

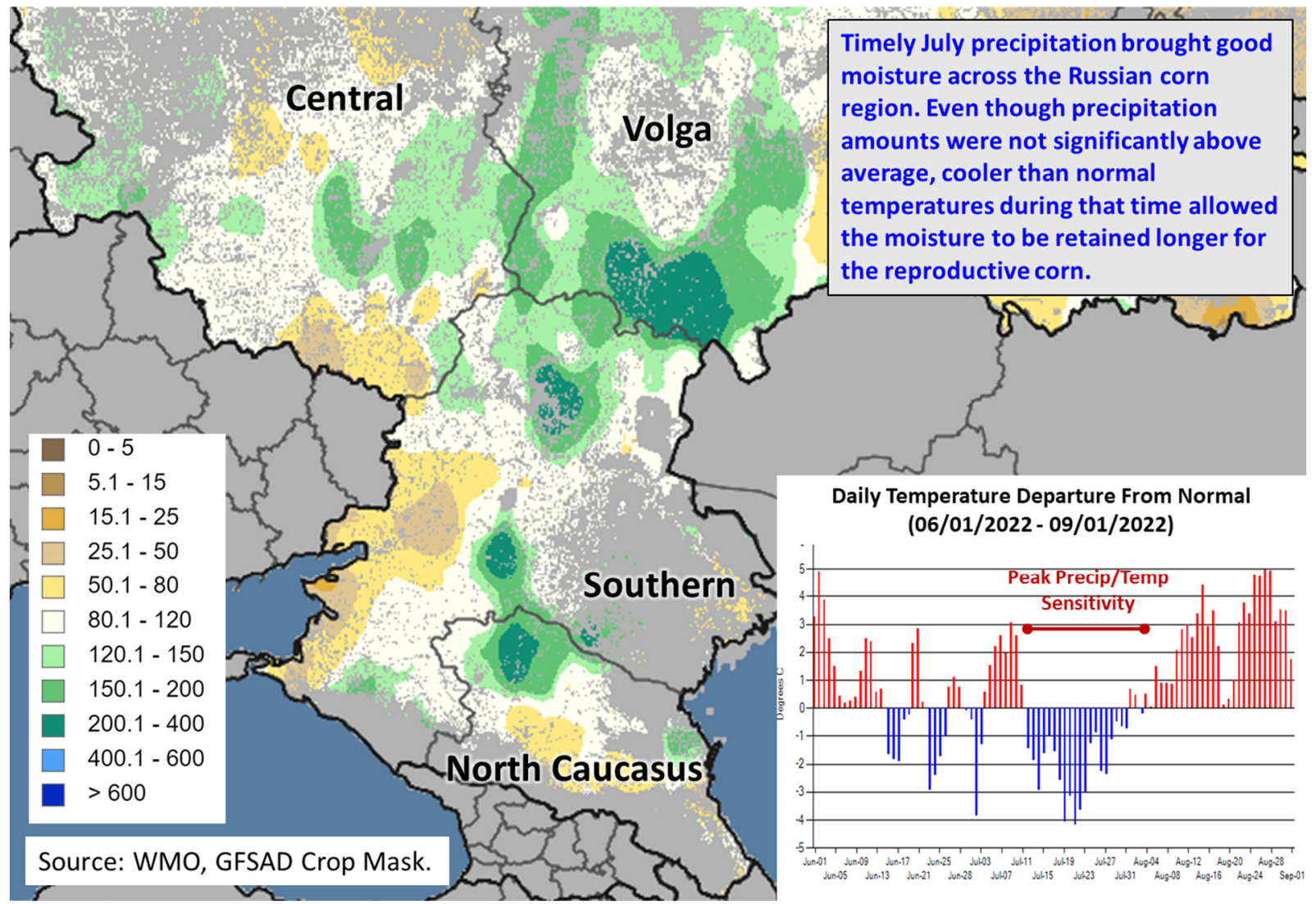

Russia's Statistical Agency, Rosstat, released its final 2022 numbers for all major crops in midMarch of 2023. Rosstat suggests a record yield for the MY 2022/23 corn crop. This season, corn harvest was substantially delayed due to abnormally wet October and November. Despite this lateseason setback, yields marked year-to-year improvements across all major corn producing districts. Timely July precipitation brought good moisture across the Russian corn region. Even though precipitation amounts were not significantly above average, cooler than normal temperatures during that time allowed the moisture to be retained longer for the reproductive corn. As a result, this season's vegetation response tracked above the average during key yield formation stages. (For more information, please contact Iliana.Mladenova@usda.gov.)

\section{South Africa Corn: Bumper Crop from Beneficial La Niña Rains}

South Africa's marketing year 2022/23 corn production is forecast at 16.7 million metric tons (mmt), unchanged from last month but up $0.6 \mathrm{mmt}$ ( 3 percent) from last year. The USDA production forecast includes output from both developing and commercial sectors, with the commercial sector accounting for approximately 97 percent of the total crop. Area is estimated at 3.0 million hectares (mha), unchanged from last month and nearly the same area as last year. Yield is forecast at 5.57 metric tons per hectare, unchanged from last month but up 4 percent from last year and up 8 percent from the 5-year average. Yields are expected to be higher than last year due to less waterlogging in regions with a high-water table. 


\section{South Africa Corn Production}

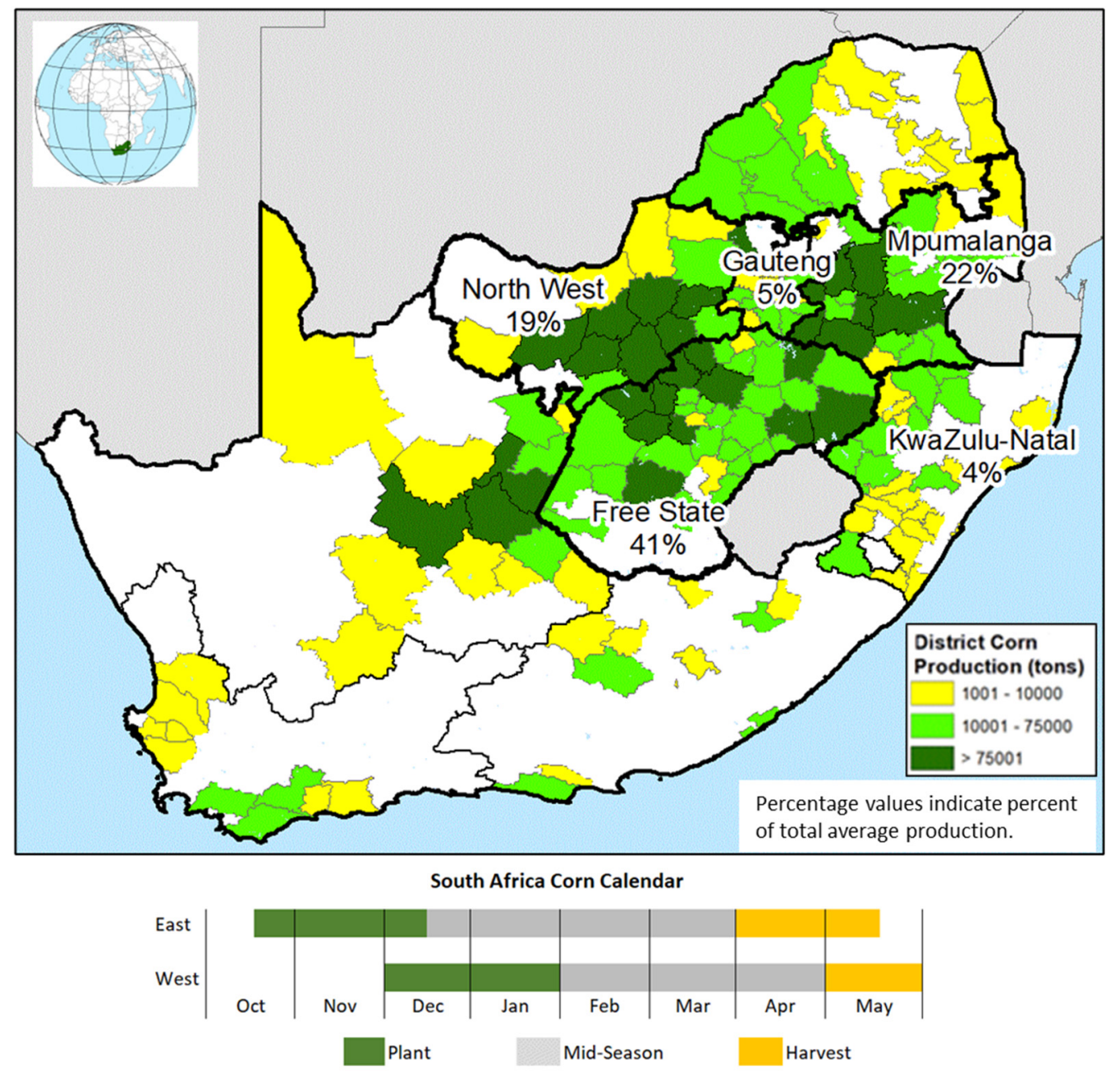

Source: Average production percentages are from South Africa's Crop Estimates Committee (2011-15), and district production data is from the Census of Commercial Agriculture, Statistics South Africa (2007)

FAS personnel traveled in South Africa's primary producing provinces in mid-March and interviewed representatives from the major grain cooperatives. Crop analysts from the cooperatives reported that beneficial La Niña rains from November 2022 through March 2023 allowed farmers to plant earlier than normal, and seasonal weather was most favorable in the Free State province where 41 percent of total crop is produced. Record or near record yields are expected in the Free State province and trend yields are expected for Mpumalanga (22 percent of total) and North West (19 percent) provinces. Satellite-derived Normalized Difference Vegetation Index (NDVI) and the Percent of Average Seasonal Greenness (PASG) indices confirmed early crop establishment, and good to excellent crop conditions by late March for all primary producing provinces. 


\section{South Africa Corn Yields}

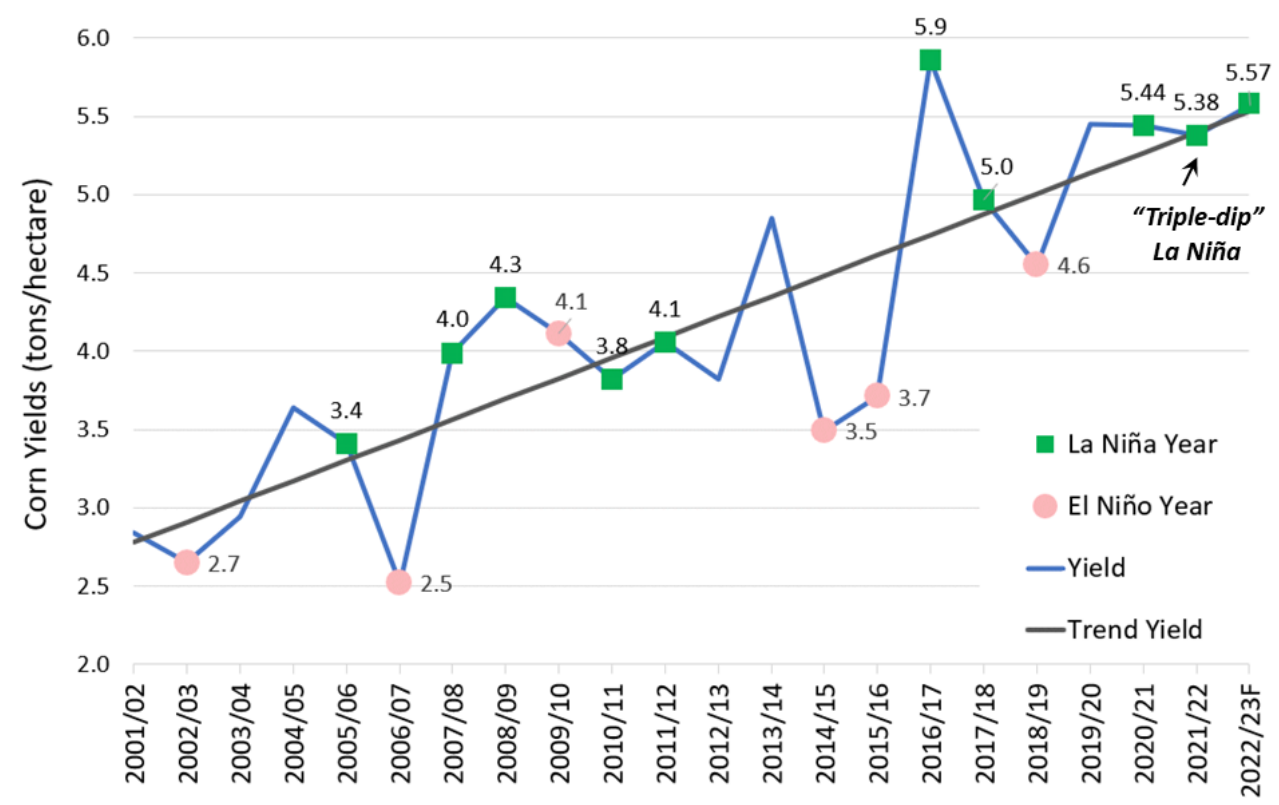

Source: USDA PSD Online

Corn reaches maturity during April and air-dried in the fields until the moisture content is low enough for harvest. The bulk of the harvest ( 87 percent) is typically delivered to the grain silos from May through August. (For more information, please contact Curt.Reynolds@usda.gov.)

\section{Ethiopia Wheat: Record Production from Favorable Rains and Increase in Irrigated Wheat Area}

Ethiopia wheat production for marketing year 2022/23 is estimated at a record 7.0 million metric tons (mmt), up $1.3 \mathrm{mmt}$ (23 percent) from last month and up $1.5 \mathrm{mmt}$ (27 percent) from last year. Harvested area is estimated at a record 2.3 million hectares (mha), up 0.3 mha (15 percent) from last month and up 0.4 mha (18 percent) from last year. Yield is estimated at a record 3.0 metric tons per hectare, up 8 percent from last year and up 6 percent from the 5-year average. Wheat yields were above average due to well-distributed rainfall received in the main highland production zones and increased wheat area under irrigation. 


\section{Ethiopia Wheat Production by District (Woreda)}

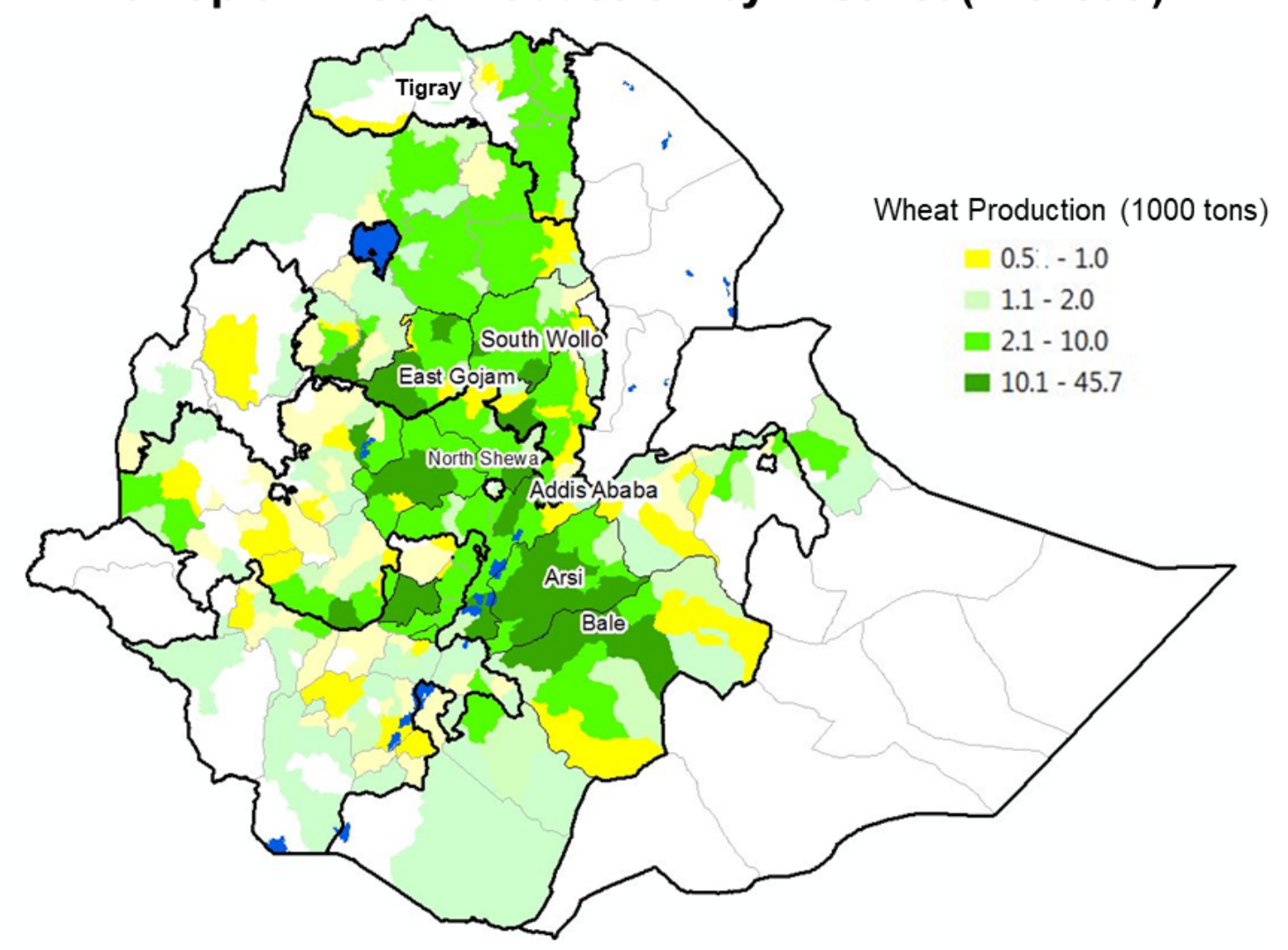

Source:

Atlas of the Ethiopian Rural Economy (2006)

Wheat is an important food staple crop in Ethiopia, and it comprises about 18 percent of the country's total cereal production. Wheat yields almost tripled during the past two decades due to favorable government initiatives that adopted improved seed varieties, increased fertilizer distribution, and expanded agriculture extension in rural areas. The Government of Ethiopia also implemented an ambitious wheat self-sufficiency plan by expanding wheat area with irrigation farming. Increases in irrigated wheat area were achieved by rotating wheat with irrigated cotton and replanting old sugarcane farms in the northern and southern lowlands with irrigated wheat. 


\section{Ethiopia Wheat Yields}

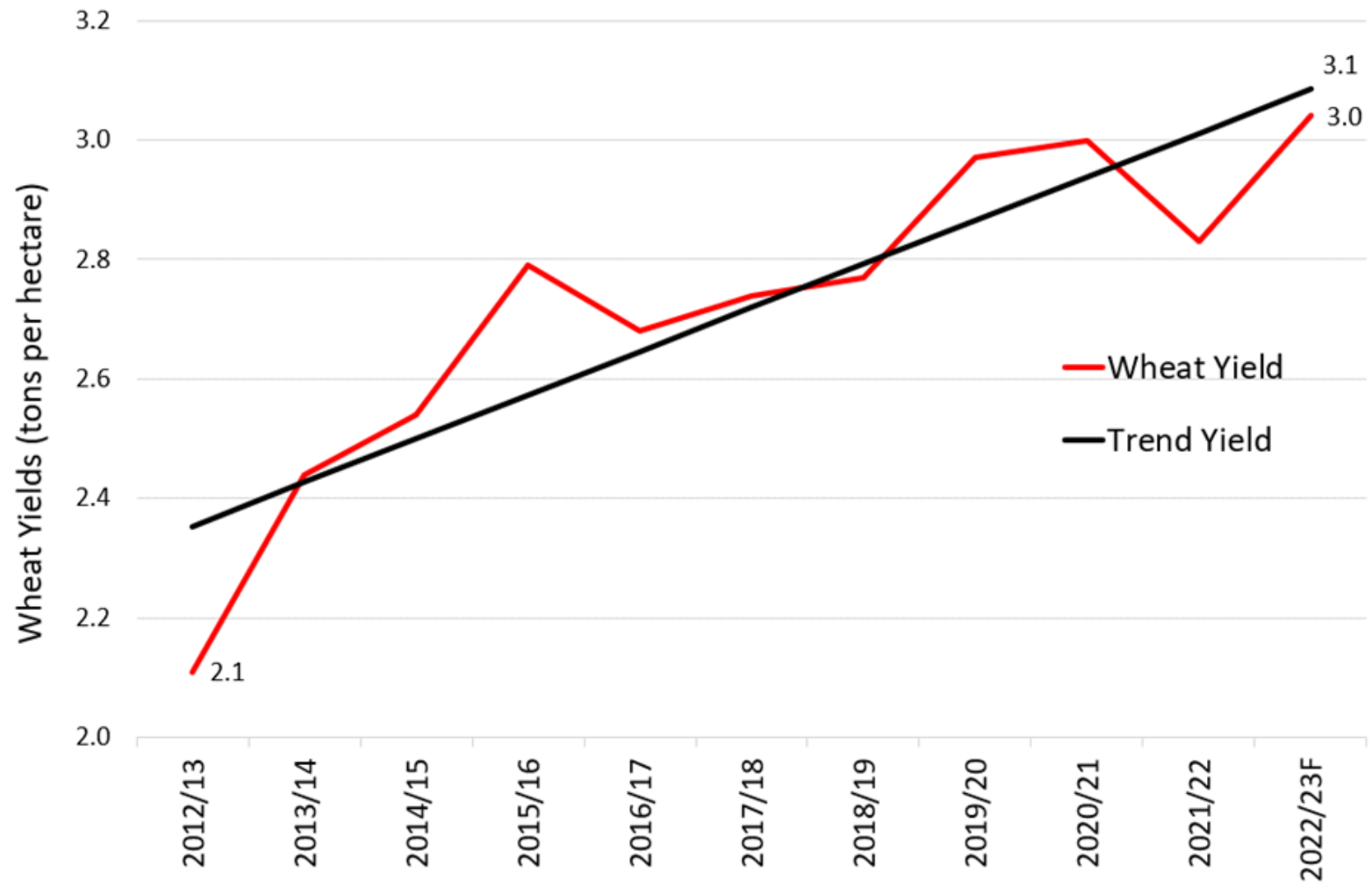

Source: USDA PSD Online

Ethiopia is the largest wheat producer in Sub-Saharan Africa, where wheat is mostly grown under rainfed conditions on small farms in the southern and central highlands. Wheat in Ethiopia is planted during the main meher rainy season from June to August and harvested from October through January. The northern Tigray region is currently facing a food security crisis after a conflict between federal and regional forces in early November displaced thousands of people. The Tigray region is chronically food insecure, and it produces approximately five percent of the country's wheat output. (For more information, please contact Curt.Reynolds@usda.gov.) 


\section{Iraq Rice: Production Down as Water Shortages Result in Lower Area and Yield}

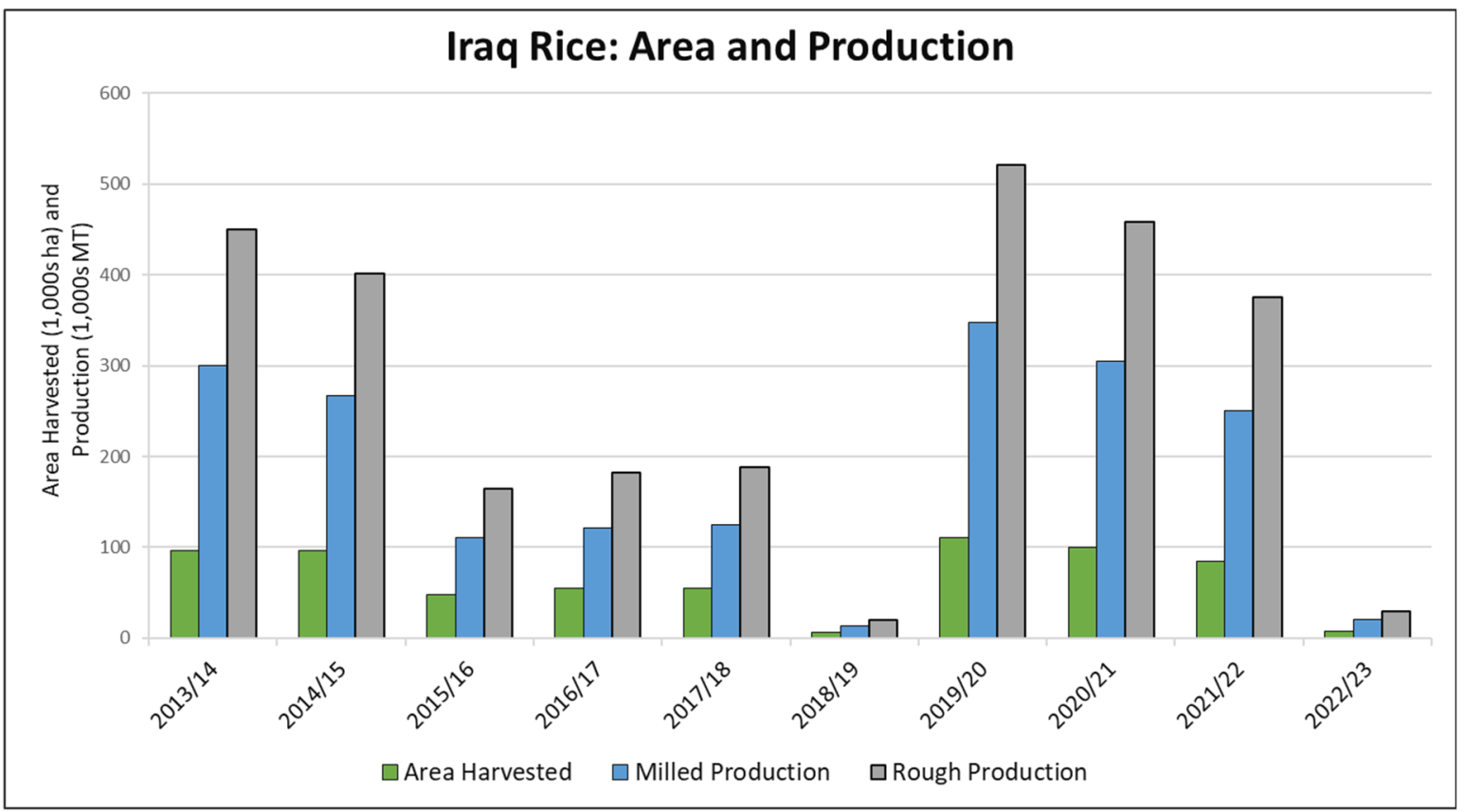

Source: USDA PSD Online

USDA estimates Iraq rice production for marketing year (MY) 2022/23 at 20,000 metric tons (milled basis), down 92 percent from last month and last year, and 90 percent below the 5-year average. Harvested area is estimated at 8,000 hectares, down 90 percent from last month, 91 percent from last year, and 89 percent below the 5-year average. Yield (rough basis) is estimated at 3.75 tons per hectare, down 18 percent from last month, 15 percent from last year, and 8 percent below the 5-year average.

\section{Iraq Water Availability Decreased in 2022}

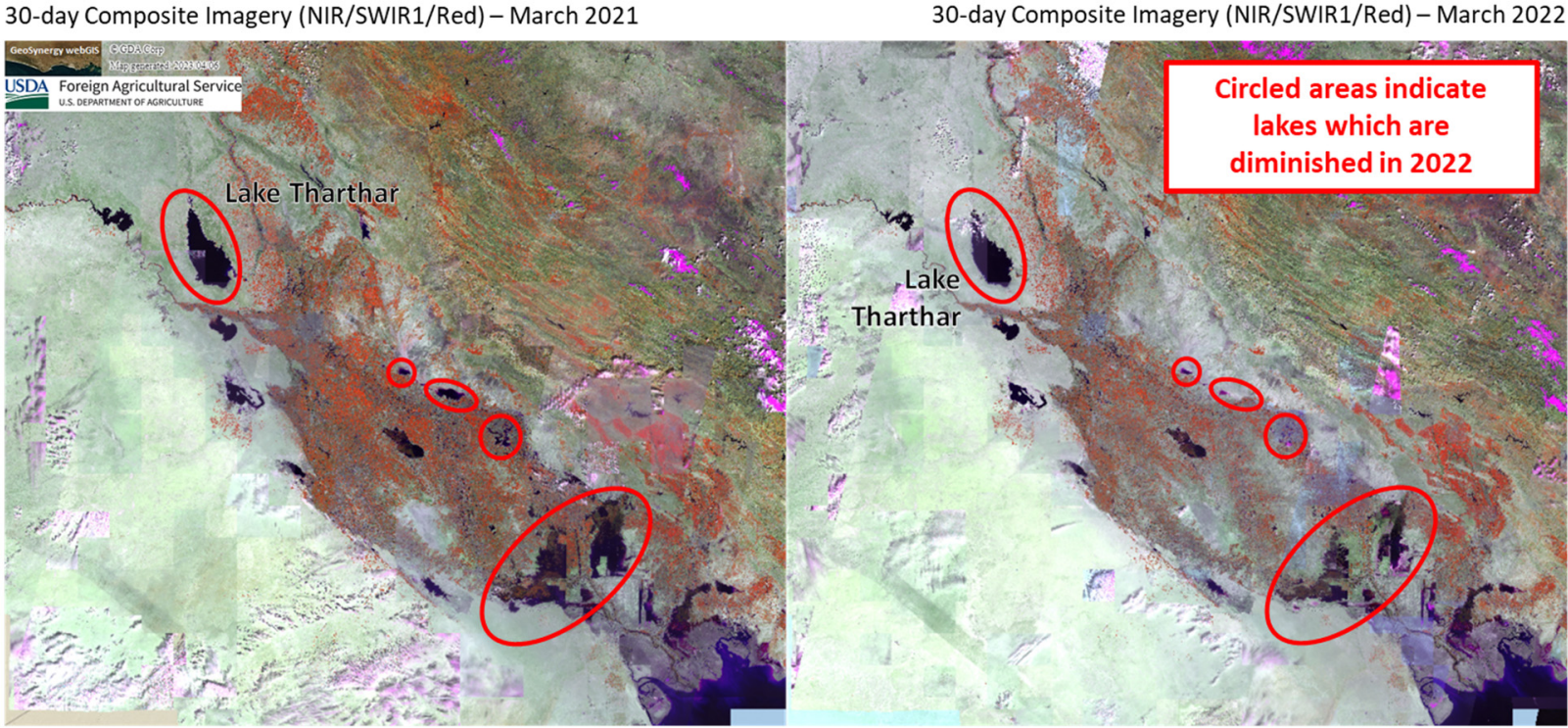

Source: GDA Corp, Sentinel-2/Landsat-8 (10m) 30-day composite imagery 
Precipitation in Iraq was below normal in 2021 and 2022 resulting in critical shortages of water storage used for irrigation, leading into the MY 2022/23 planting season, which began with planting in April and May. Satellite imagery from March of 2022 indicated diminished lakes and reservoirs throughout the primary rice-growing areas of southeastern Iraq. Of particular interest is Lake Tharthar, an artificial reservoir built in 1956 to hold floodwaters from the Tigris River to later discharge into both the Tigris and Euphrates during dry seasons. Imagery indicates smaller lake area in March 2022 than during the same period in 2021.

\section{Lake Tharthar Water Level Variations from Altimetry}

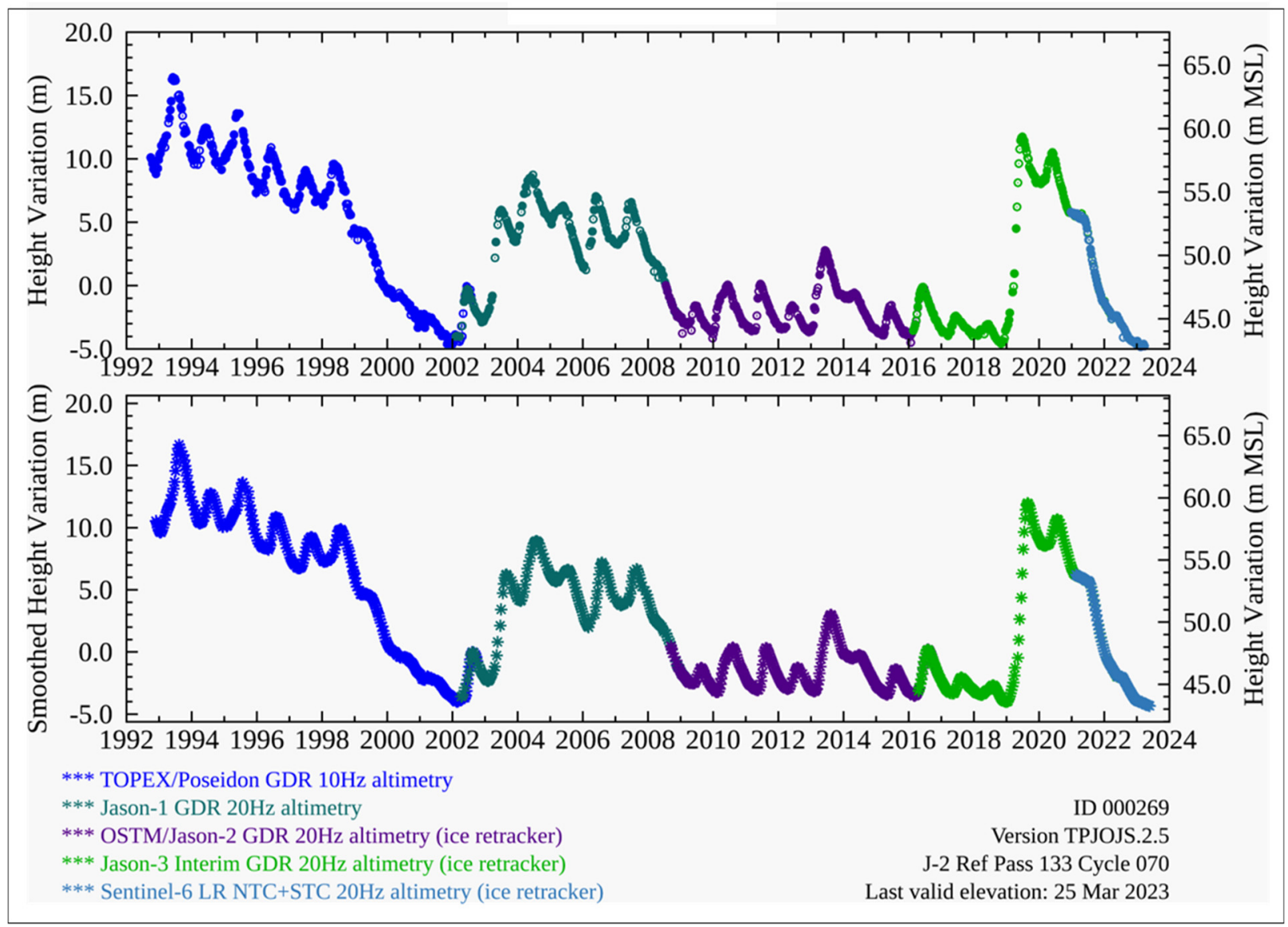

Source: USDA/NASA Global Reservoirs and Lakes Monitor (G-REALM)

In response to the water shortages, the Iraqi government reduced area allocated for rice cultivation by as much as 97 percent, according to government and industry reporting. Altimetry data indicates water levels in Lake Tharthar are well-below normal, and similar to 2018, the last time the government placed severe restrictions on rice cultivation. USDA also expects lower yields given the drought conditions that persisted throughout the growing season of 2022. (For more information, please contact Aaron.Mulhollen@usda.gov.) 


\section{Argentina Soybeans: Production Down 39 Percent from Last Year}

Argentina soybean production for marketing year 2022/23 is estimated at 27.0 million metric tons, down 18 percent from last month, and down 38 percent from last year. Soybean yield is estimated at 1.80 tons per hectare, down 15 percent from last month, and down 35 percent from last year. Harvested area is estimated at 15.0 million hectares, down 3 percent from last month, and down 6 percent from last year.

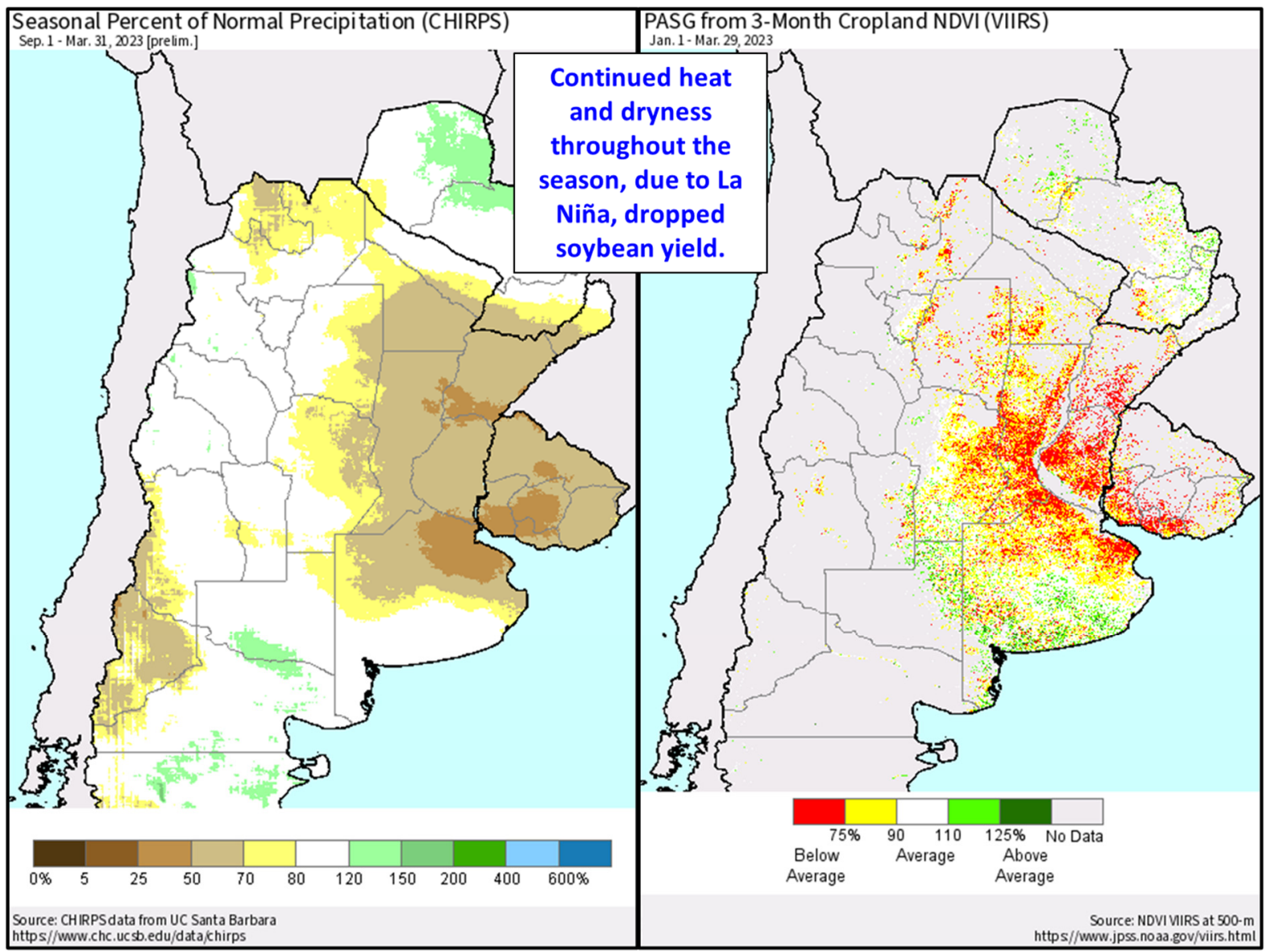

Yield is down month-to-month because of dry and hot conditions throughout March. Rains that occurred during the latter half of March were too late to provide benefit to the soybean crop. Harvest has just begun, and early yields are below average. FAS Buenos Aires, who conducted crop travel through major soybean areas during March, noted the lower-than-average yields during harvest. Harvest will continue until early June. (For more information, please contact Katie.McGaughey@usda.gov.) 


\section{Uruguay Soybeans: Production Down 63 Percent from Last Year}

Uruguay soybean production for marketing year 2022/23 is estimated at 1.2 million metric tons, down 43 percent from last month, and down 63 percent from last year. Soybean yield is estimated at 1.33 tons per hectare, down 27 percent from last month, and down 52 percent from last year. Harvested area is estimated at 0.9 million hectares, down 22 percent from last month and from last year.

\section{Uruguay: CHIRPS 10-day Cumulative Precipitation}

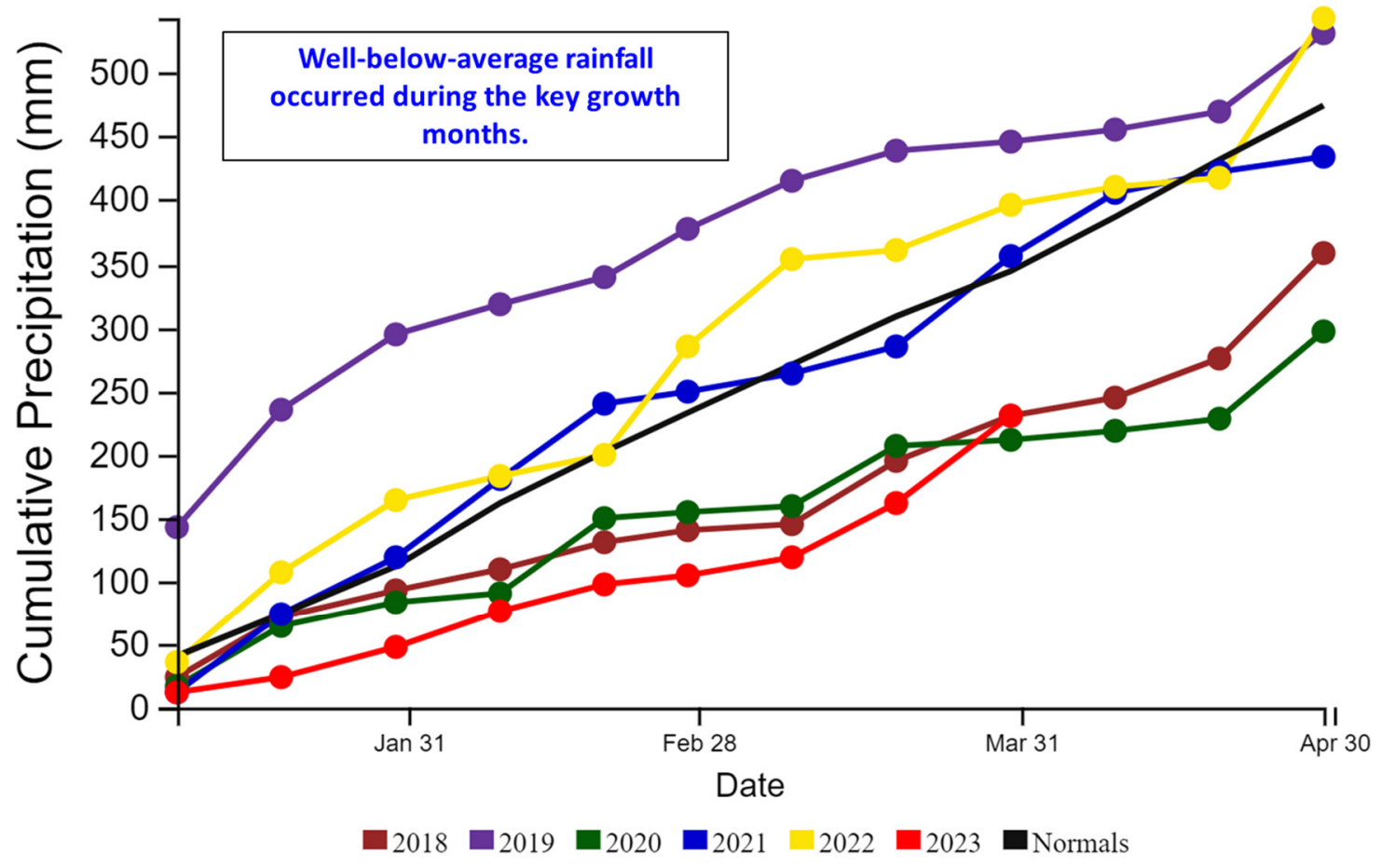

Source: UCSB CHIRPS

https://www.chc.ucsb.edu/data/chirps

Yield and area are both down month-to-month because of dry and hot conditions throughout the growing season. Rains that occurred during late March were too late to provide benefit to the soybean crop since harvest had already begun. FAS Buenos Aires, who conducted crop travel through major soybean areas during March, noted the lower-than-average yields and higher-thannormal abandonment information during harvest. Harvest will continue until mid-May. (For more information, please contact Katie.McGaughey@usda.gov.) 


\section{MY 2022/23 Soybean Yields Compared to the 5 Year Average}

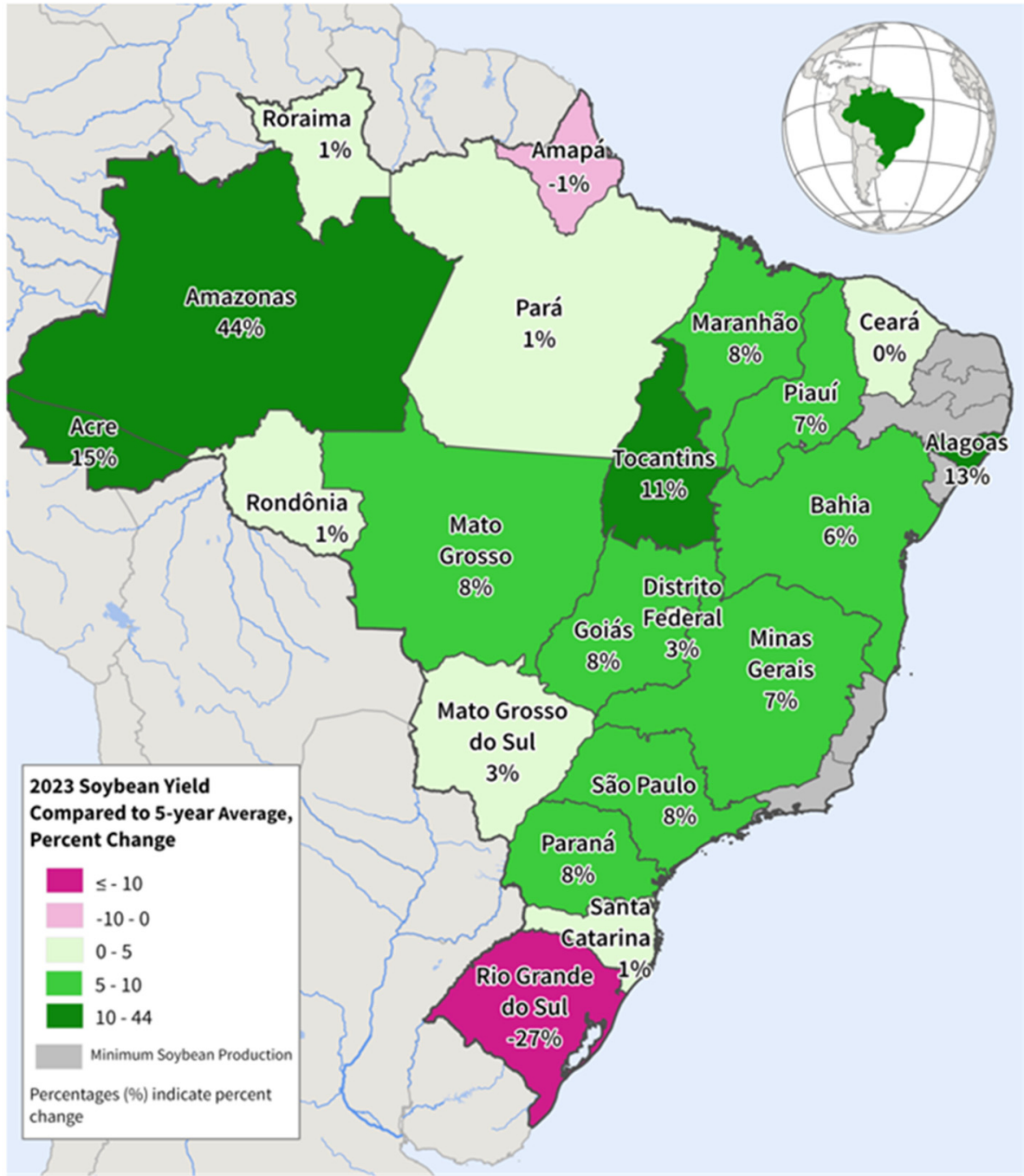

Sources: Average soybean yields for 2018-2022 from Série histórica das Safras by CONAB. April 2023 state yields substituted from Mato Grosso (IMEA), Paraná (DERAL), and Rio Grande do Sul (EMATER-RS) reports.

Brazil soybean production for marketing year (MY) 2022/23 is estimated at a record 154.0 million metric tons (mmt), higher by $1.0 \mathrm{mmt}$ (1 percent) from last month, and higher by $24.0 \mathrm{mmt}$ (18 percent) from last year. Harvested area is estimated at a record 43.7 million hectares (mha), up 0.3 mha (1 percent) from last month and up $2.1 \mathrm{mha}(5$ percent) from last season. Yield is estimated at 3.52 tons per hectare ( $\mathrm{t} / \mathrm{ha}$ ), slightly below last month and up 12 percent from last year. A revision was made to MY 2021/22 with area raised to $41.6 \mathrm{mha}$, production to $130.5 \mathrm{mmt}$ with a yield of $3.14 \mathrm{t} / \mathrm{ha}$. For MY 2022/23, soybean production in the large center-west states have compensated for problems in Rio Grande do Sul. 


\section{Percent of Average Seasonal Greenness (PASG) from January to March Displays Vegetation during 3 Consecutive Years of La Niña}

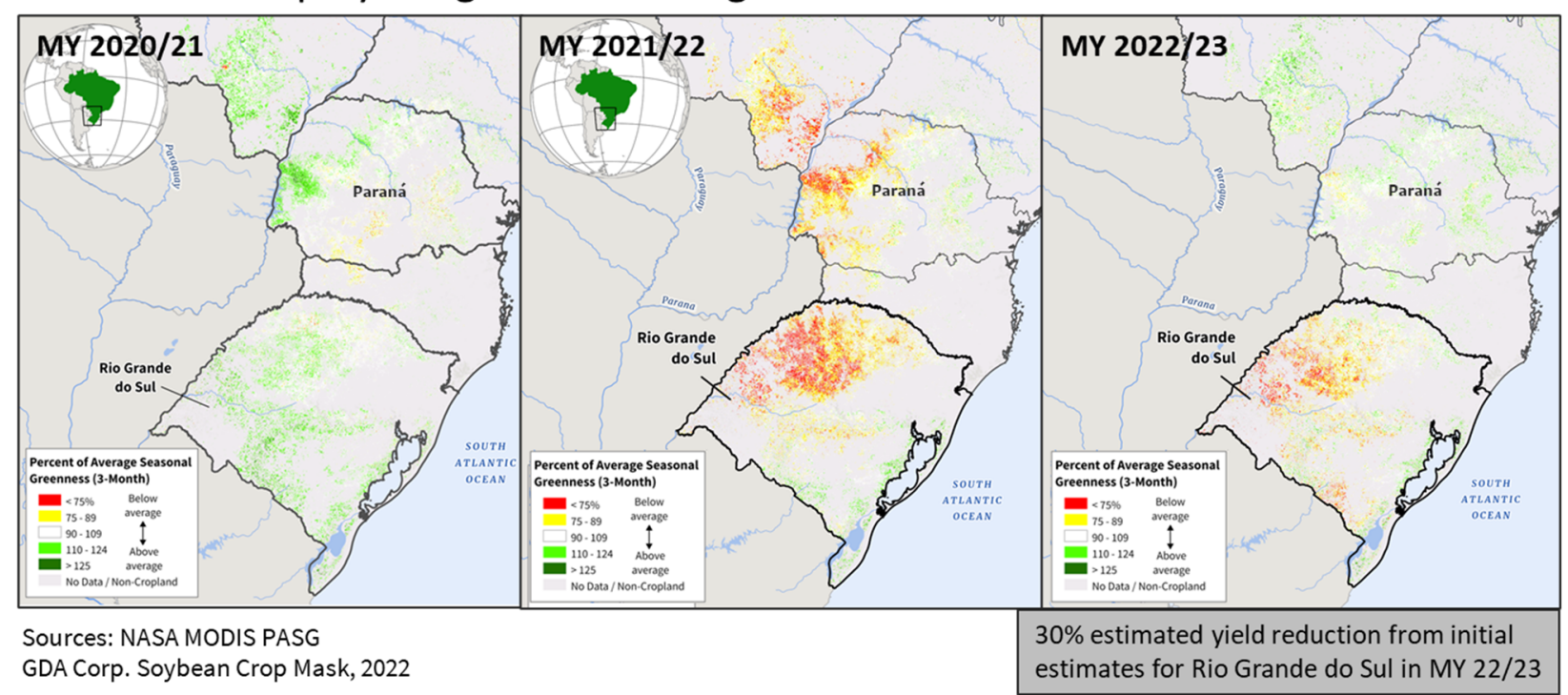

Harvest was delayed in several key center-west states due to heavy rains. Nationally, about 75 percent of the soybean crop has been harvested versus 81 percent last year in early April. The largest producer, Mato Grosso, completed harvest and reports a record crop of $44.3 \mathrm{mmt}$ on a record area of 12.0 mha per Instituto Mato-Grossense de Economia Agropecuária (IMEA). Second ranked Paraná is also reporting a record output $(\sim 22 \mathrm{mmt})$ according to the Paraná Department of Agriculture (SEAB/DERAL). The spot for the number three producer this year is taken up by Goiás $(\sim 17.3 \mathrm{mmt})$. Meanwhile, the usual number three producer, Rio Grande do Sul, experienced another season riddled by drought which reduced yields by 30 percent resulting in a production loss of roughly $6 \mathrm{mmt}$ (as reported by The Technical Assistance and Rural Extension of Rio Grande do Sul, EMATER-RS). In the Northeast, harvest stands at 69 percent with yields reported to be well above average. (For more information, please contact Sunita.Yadav-Pauletti@usda.gov.) 


\section{Brazil Cotton: Estimated Output for MY 2022/23 Reduced}

Brazil's marketing year (MY) 2022/23 cotton production is forecast at 13.0 million 480-pound bales, lower by 0.3 million bales ( 2 percent) from last month, and up by 1.3 million bales (11 percent) from last year. Harvested cotton area is estimated at 1.6 million hectares (mha), unchanged from last month, and up 2 percent from last year. Cotton yield is forecast at 1,736 kilograms per hectare $(\mathrm{kg} / \mathrm{ha})$, roughly 2 percent lower than last month, but 9 percent above the previous year.

\section{Cotton Area: Brazil and Key States of Mato Grosso and Bahia}

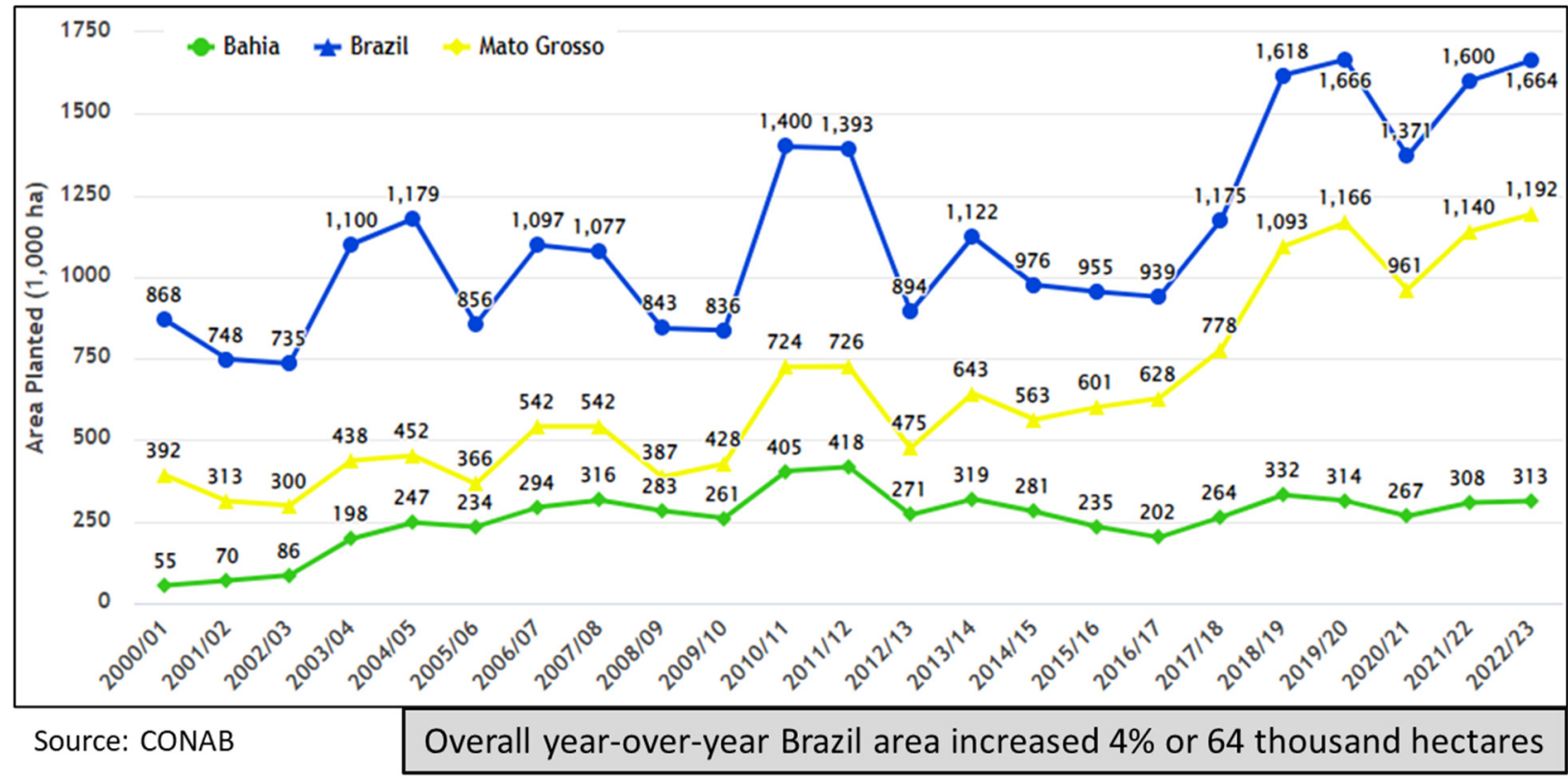

The full-season cotton crop was planted from late November to early February in Bahia (20 percent of national production). The second-season cotton crop in Mato Grosso (70 percent of national production) was planted with a delay this season due to excessive rainfall delaying the soybean harvest. However, this delay was not as significant as the one 2 years ago in MY 2020/21. The Instituto Mato-Grossense de Economia Agropecuária (IMEA) reports an area reduction this season of $0.03 \mathrm{mmt}$. 


\section{Bahia Cotton Precipitation Anomaly and Yield Association}

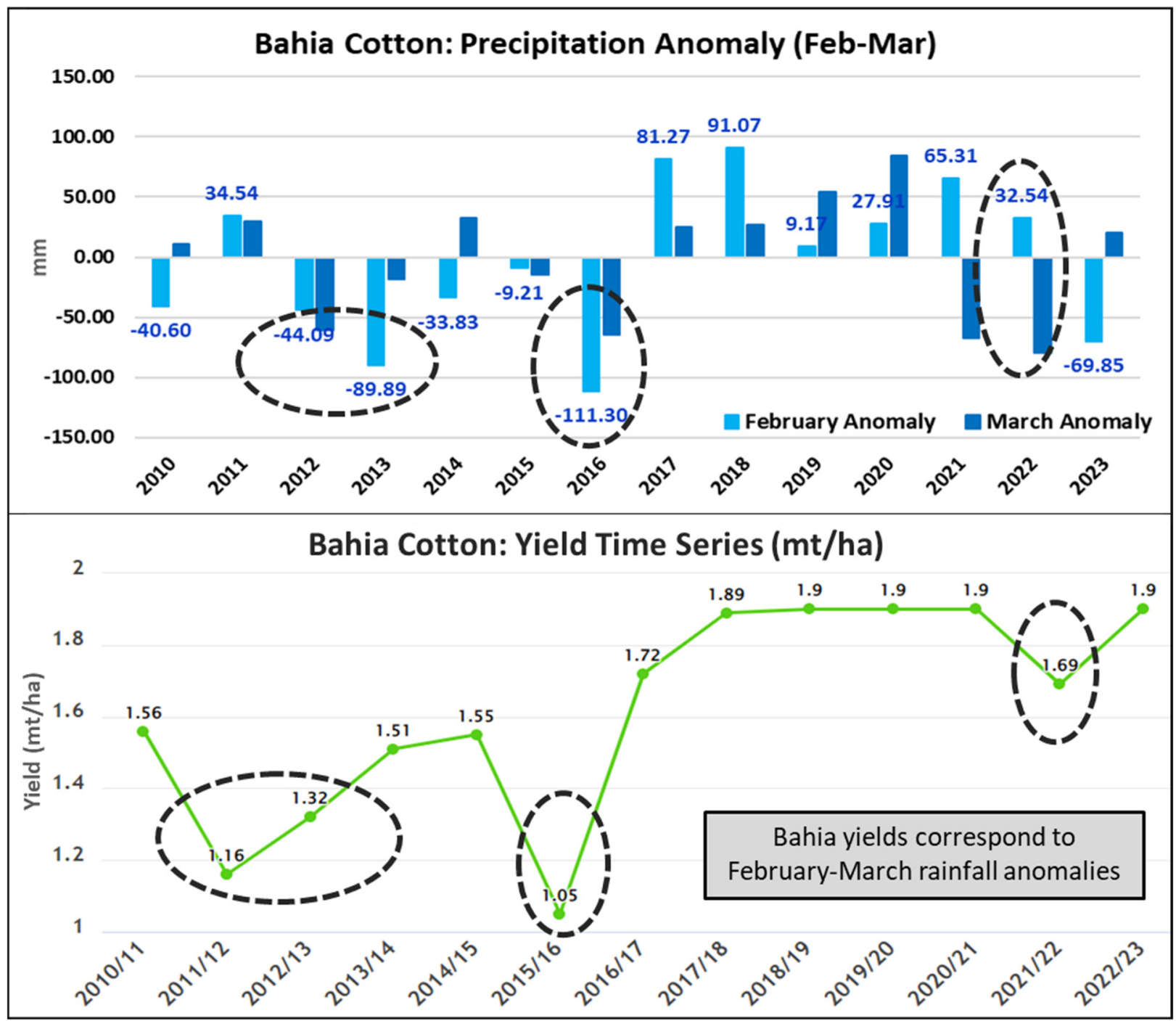

Sources: UCSB CHIRPS, CONAB

Similar to last year, rainfall in western Bahia, where most cotton is cultivated, once again was below normal in February when the crop was in critical development stages. The difference from last year is that the rainfall volume recovered this March bringing some relief to crops, whereas it reduced further last March. Nevertheless, the yield expectations are reduced as the rains may not reverse some of the crop damage. Weather in Mato Grosso has been favorable for the crop thus far. The majority of the cotton will be harvested from June to August. (For more information, please contact Sunita.Yadav-Pauletti@usda.gov.) 


\section{China Cotton: Production Increased Due to Record Yields}

USDA estimates China's marketing year (MY) 2022/23 cotton production at 30.5 million 480pound bales (6.6 million metric tons), up 1.0 million bales or 3 percent from last month, up 3.7 million bales or 14 percent from last year, and 9 percent from the 5-year average of 27.9 million bales. Harvested area is estimated at 3.05 million hectares (mha), same as last month but down 2 percent from last year, down 8 percent from the 5-year average of 3.3 mha. Yield is estimated at a record 2,177 kilograms per hectare $(\mathrm{kg} / \mathrm{ha})$, up 3 percent from last month, 16 percent from last year, and 18 percent above the 5 -year average of $1,842 \mathrm{~kg} / \mathrm{ha}$.

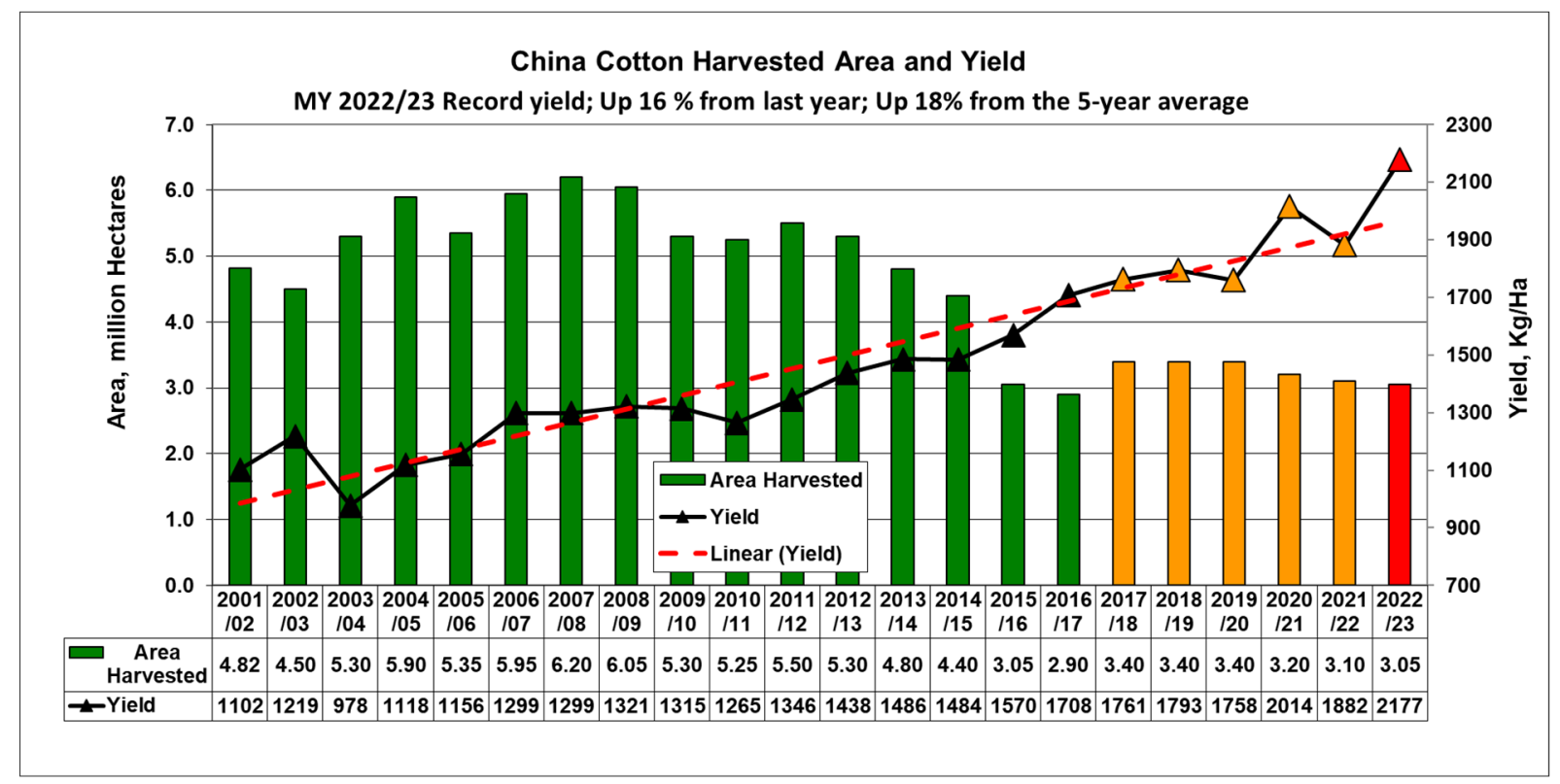

Source: USDA PSD Online

Yield is significantly above the long-term trend due to the increasing quantity of cotton produced in Xinjiang province, where yield is nearly that elsewhere in the country, especially the North China Plain. Approximately 90 percent of total China cotton is now produced in Xinjiang province. Xinjiang's daily cotton processing data of inspection, ginning, and classing are higher compared to the same period last year, September through early April. The processing data published by China Fiber Quality Monitoring Center and China National Cotton Exchange continues to provide evidence indicating higher-than-expected production compared to the previous years. Most of the cotton estimates from major industry sources are also indicating higher-than-expected yields resulting in significant adjustment of production numbers. 


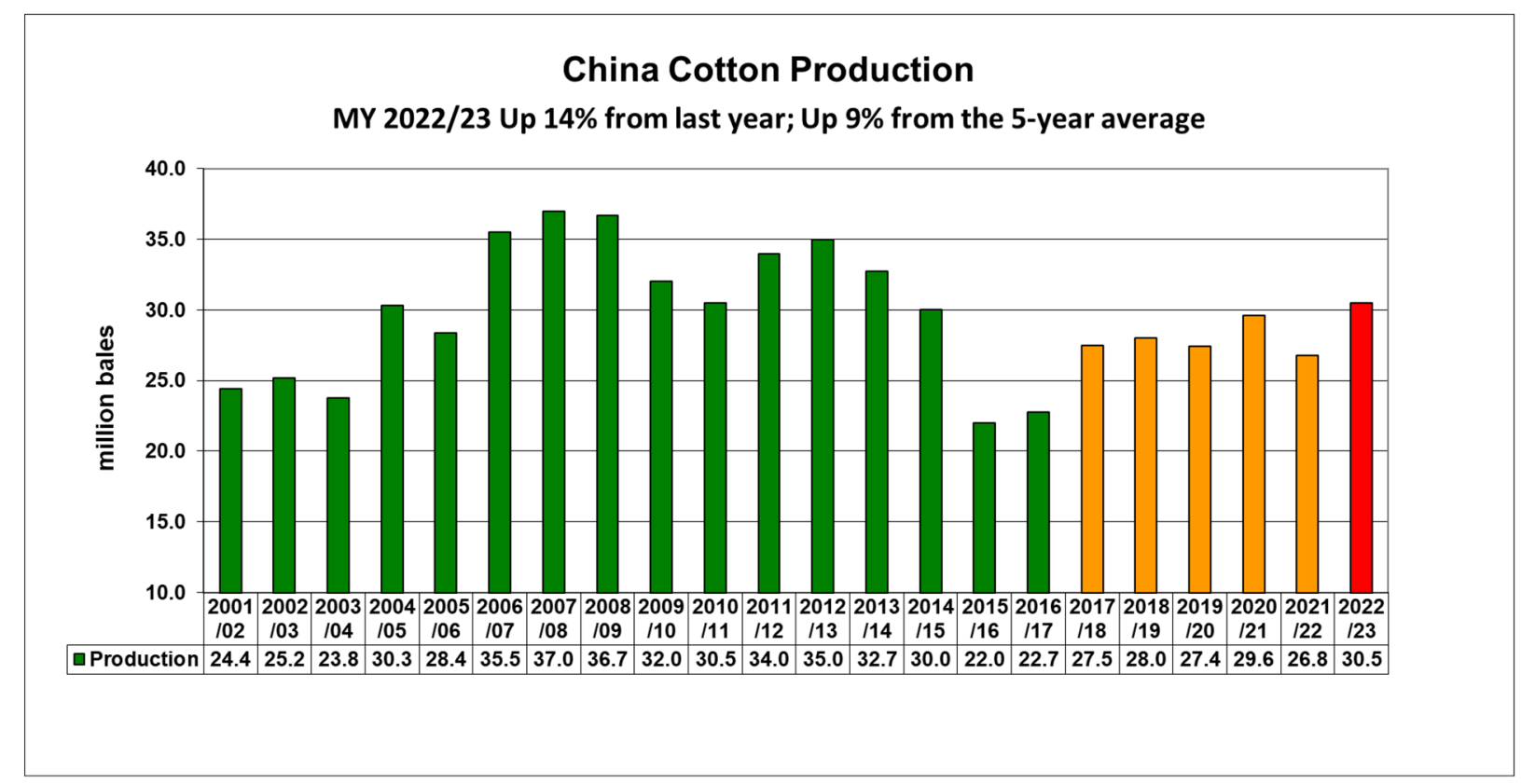

Source: USDA PSD Online

Harvest was completed in October; the typical cotton growing season is April to October. In Xinjiang, planting starts in April while on the North China Plain, the Yellow River and Yangtze River basins, planting begins in late April and extends through May. The MY 2022/23 season was characterized by favorable conditions, good irrigation water availability, and near-optimal temperatures throughout a major part of the season. These conditions facilitated rapid planting and early crop establishment in Xinjiang resulting in above-average yields. In addition to the favorable planting conditions, farmers were also encouraged by the government's continuation of the target price-based cotton subsidy. On March 26, 2020, China's National Development and Reform Commission (NDRC) published a notice stating that the target price-based subsidy will continue through 2023. The year-to-year cotton national area decline was due to decreases in the Yangtze and Yellow River basins, which offset an increase in Xinjiang. However, the significant year-toyear yield increase more than offset the planted area reduction, resulting in increased production. (For more information, please contact Dath.Mita@usda.gov.) 


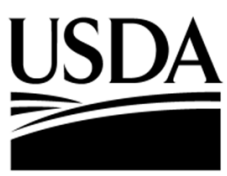

\section{World Agricultural Production \\ U.S. Department of Agriculture}

Foreign Agricultural Service / Global Market Analysis

International Production Assessment Division (IPAD)

Ag Box 1051, Room 4630, South Building

Washington, DC 20250-1051

https://ipad.fas.usda.gov/

Telephone: (202) 720-1662 Fax: (202) 720-1158

This report uses information from the Foreign Agricultural Service's (FAS) global network of agricultural attachés and counselors, official statistics of foreign governments and other foreign source materials, and the analysis of economic data and satellite imagery. Estimates of foreign area, yield, and production are from the International Production Assessment Division, FAS, and are reviewed by USDA's Inter-Agency Commodity Estimates Committee. Estimates of U.S. area, yield, and production are from USDA's National Agricultural Statistics Service. Numbers within the report may not add to totals because of rounding. This report reflects official USDA estimates released in the World Agricultural Supply and Demand Estimates (WASDE-584), April 11, 2023.

The FAS International Production Assessment Division prepared this report. The next issue of World Agricultural Production will be released after 12:00 p.m. Eastern Time, May 12, 2023.

\section{Conversion Table}

$\underline{\text { Metric tons to bushels }}$

\begin{abstract}
Wheat, soybeans Corn, sorghum, rye Barley
\end{abstract}

Oats

Cotton

Rice

1 hectare

1 kilogram

$\begin{array}{ll}= & \mathrm{MT} * 36.7437 \\ = & \mathrm{MT} * 39.36825 \\ = & \mathrm{MT} * 45.929625 \\ = & \mathrm{MT} * 68.894438\end{array}$

Metric tons to $480-1 b$ bales

$=$

$\mathrm{MT} * 4.592917$

Metric tons to hundredweight

$\mathrm{MT} * 22.04622$

Area \& weight

$\begin{array}{ll}= & 2.471044 \text { acres } \\ = & 2.204622 \text { pounds }\end{array}$


For further information, contact:

\section{U.S. Department of Agriculture \\ Foreign Agricultural Service \\ Global Market Analysis}

International Production Assessment Division

Ag Box 1051, Room 4630, South Building

Washington, DC 20250-1051

Telephone: (202) 720-1662

Fax: (202) 720-1158

\section{GENERAL INFORMATION}

\section{Senior Director}

Director

Administrative Assistant

USDA Remote Sensing Advisor

Sr. Analyst/Technical Lead

Sr. Analyst/ Satellite Imagery

Archives Manager/Technical Lead

Sr. Analyst/Technical Lead

GIS Mapping/Imagery Analyst

WAP Coordinators

$\begin{array}{lll}\begin{array}{l}\text { Ronald Frantz } \\ \text { Robert Tetrault } \\ \text { Vacant }\end{array} & \begin{array}{l}202-720-4056 \\ \text { 202-720-1071 }\end{array} & \begin{array}{l}\text { ronald.frantz@usda.gov } \\ \text { robert.tetrault@usda.gov }\end{array} \\ \begin{array}{l}\text { Vacant } \\ \text { Curt Reynolds, Ph.D. }\end{array} & 202-690-0134 & \begin{array}{l}\text { curt.reynolds@usda.gov } \\ \text { Dath Mita, Ph.D. }\end{array} \\ \begin{array}{lll}\text { dath.mita@usda.gov } \\ \text { Jim Crutchfield }\end{array} & 202-720-7339 & \text { james.crutchfield@usda.gov } \\ \text { Lisa Colson } & 202-720-6652 & \begin{array}{l}\text { lisa.colson@usda.gov } \\ \text { Ifeoma Collins }\end{array} \\ \text { Aaron Mulhollen } & 202-720-0240 & \text { ifeoma.collins@usda.gov } \\ \text { aaron.mulhollen@usda.gov }\end{array}$

\section{COUNTRY-AND REGION-SPECIFIC INFORMATION}

\begin{tabular}{|c|c|c|c|}
\hline $\begin{array}{l}\text { South America, Argentina, Venezuela, } \\
\text { and Colombia }\end{array}$ & Katie McGaughey & $202-720-9210$ & katie.mcgaughey@usda.gov \\
\hline $\begin{array}{l}\text { Western and Central Europe, } \\
\text { and North Africa }\end{array}$ & Bryan Purcell & $202-690-0138$ & bryan.purcell@usda.gov \\
\hline $\begin{array}{l}\text { Russia, Ukraine, Kazakhstan, Moldova, } \\
\text { and Belarus }\end{array}$ & Iliana Mladenova, Ph.D. & $202-690-2315$ & iliana.mladenova@usda.gov \\
\hline $\begin{array}{l}\text { South Asia, Sri Lanka } \\
\text { Bangladesh, India, and Nepal }\end{array}$ & Arnella Trent & $202-720-0881$ & arnella.trent@usda.gov \\
\hline East Asia, China, and Japan & Dath Mita, Ph.D. & $202-720-7339$ & dath.mita@usda.gov \\
\hline $\begin{array}{l}\text { Sub-Saharan Africa, } \\
\text { Nigeria and South Africa }\end{array}$ & Curt Reynolds, Ph.D. & 202-690-0134 & curt.reynolds@usda.gov \\
\hline $\begin{array}{l}\text { S.E. Asia, Indonesia, Thailand, Malaysia, } \\
\text { Cambodia and Vietnam }\end{array}$ & Justin Jenkins & 202-720-0419 & justin.jenkins@usda.gov \\
\hline $\begin{array}{l}\text { Central America, Mexico, } \\
\text { and Western United States }\end{array}$ & Ifeoma Collins & $202-720-0240$ & ifeoma.collins@usda.gov \\
\hline $\begin{array}{l}\text { Australia, New Zealand, Pakistan } \\
\text { Papua New Guinea and South Pacific Isla }\end{array}$ & $\begin{array}{l}\text { Jim Crutchfield } \\
\text { ds }\end{array}$ & $202-690-0135$ & james.crutchfield@usda.gov \\
\hline $\begin{array}{l}\text { Middle East, Central Asia, Afghanistan } \\
\text { Iraq, Iran, and Syria }\end{array}$ & Aaron Mulhollen & 202-690-0131 & aaron.mulhollen@usda.gov \\
\hline $\begin{array}{l}\text { Canada, Caribbean, } \\
\text { Eastern United States }\end{array}$ & Aaron Mulhollen & 202-690-0131 & aaron.mulhollen@usda.gov \\
\hline Brazil & \multicolumn{2}{|c|}{ Sunita Yadav-Pauletti, Ph.D. } & sunita.yadav-pauletti@usda.g \\
\hline
\end{tabular}


The Foreign Agricultural Service (FAS) updates its production, supply and distribution (PSD) database for cotton, oilseeds, and grains at 12:00 p.m. on the day the World Agricultural Supply and Demand Estimates (WASDE) report is released. This circular is released by 12:15 p.m.

\title{
FAS Reports and Databases:
}

\begin{abstract}
World Agricultural Production
Current: https://www.fas.usda.gov/data/world-agricultural-production

Archive: https://usda.library.cornell.edu/concern/publications/5q47rn72z?locale=en

USDA's Foreign Agricultural Service (FAS) publishes a monthly report on crop acreage, yield and production in major countries worldwide. Sources include reporting from FAS's worldwide offices, official statistics of foreign governments, and analysis of economic data and satellite imagery. The reports reflect official USDA estimates released in the monthly World Agricultural Supply and Demand Estimates (WASDE).
\end{abstract}

\section{World Markets and Trade}

Current: https://www.fas.usda.gov/data

Archive:

https://usda.library.cornell.edu/catalog? $\%$ BBmember_of_collections_ssim $\% 5 \mathrm{D} \% 5 \mathrm{~B} \% 5 \mathrm{D}=$ Foreign + Agricultural $+\mathrm{Se}$ rvice\&locale $=$ en

USDA's Foreign Agricultural Service (FAS) publishes monthly and quarterly reports which include data on U.S. and global trade, production, consumption and stocks, as well as analysis of developments affecting world trade in oilseeds, grains, cotton, livestock and poultry. The reports reflect official USDA estimates released in the monthly World Agricultural Supply and Demand Estimates (WASDE).

\section{Global Agricultural Information Network (GAIN)} https:/gain.fas.usda.gov/ USDA's Foreign Agricultural Service (FAS) provides timely reports on foreign markets through the Global Agriculture Information Network (GAIN) database. An average of 2,000 reports are added each year, with reports going back to 1995. GAIN reports are compiled by FAS' global market intelligence network, which includes FAS foreign service officers and locally engaged staff in over 90 overseas offices world-wide. They provide on-the-ground intelligence, insight, and analysis on nearly 200 countries, delivering information on foreign agricultural markets, crop conditions, and agro-political dynamics of interest to U.S. agriculture. GAIN reports contain assessments of commodity and trade issues made by USDA staff and are not necessarily statements of official U.S. government policy.

\section{Production, Supply and Distribution (PS\&D) Online}

https://apps.fas.usda.gov/psdonline/app/index.html\#/app/home

PSD Online is the public repository for USDA's Official Production, Supply and Distribution forecast data and reports for key agricultural commodities. PSD Online data are reviewed and updated monthly by an interagency committee chaired by USDA's World Agricultural Outlook Board (WAOB). The committee consist of representatives from Foreign Agricultural Service (FAS), the Economic Research Service (ERS), the Farm Service Agency (FSA), and the Agricultural Marketing Service (AMS).

\section{EU Countries and Russia Wheat: Area and Production Estimates}

https://apps.fas.usda.gov/psdonline/app/index.html\#/app/downloads (click on PSD Datasets)

USDA's Foreign Agricultural Service (FAS) provides country-level area and production estimates for the nations of the European Union. For Russia, country-level area and production estimates are provided for Winter and Spring wheat. These datasets are reviewed and updated monthly by an interagency committee chaired by USDA's World Agricultural Outlook Board (WAOB) and can be downloaded through PSD datasets on PSD Online. 


\section{FAS Reports and Databases:}

\section{International Production Assessment (IPAD)}

https://ipad.fas.usda.gov/

USDA's Foreign Agricultural Service (FAS) maintains a dynamic global crop production portal with key information including World Agricultural Production (WAP) briefs, Commodity Intelligence Reports (CIR), geospatial applications, crop production maps at a subnational level and crop calendars for the top-20 producing countries. Additionally, Crop Explorer (https://ipad.fas.usda.gov/cropexplorer/) displays rainfall, temperature, evapotranspiration, soil moisture, snow cover, and vegetation conditions in charts and maps.

\section{USDA and NASA Global Agricultural Monitoring (GLAM)}

https://glam1.gsfc.nasa.gov/

The USDA and NASA Global Agricultural Monitoring (GLAM) system provides near real-time and science quality Moderate Resolution Imaging Spectroradiometer (MODIS) Normalized Difference Vegetation Index (NDVI) from the satellites Terra and Aqua. The public can view and retrieve MODIS 8-day composited, global NDVI satellite imagery and time series data. GLAM was developed by NASA's Global Inventory Modeling and Mapping Studies (GIMMS) group for USDA's Foreign Agricultural Service.

\section{Global Agricultural and Disaster Assessment System (GADAS)}

https://geo.fas.usda.gov/GADAS/index.html

USDA's Foreign Agricultural Service (FAS) provides the Global Agricultural and Disaster Assessment System (GADAS), a web-based Geographic Information System (GIS) tool which integrates a vast array of highly detailed earth observation data streams, particularly targeted towards agricultural and disaster assessment analysis. GADAS is an interactive website which provides analysts with a wide variety of routine geospatial products (maps, charts, tables) they require for comprehensive situational investigations and recurring assessments.

\section{Export Sales Reporting}

\section{https://apps.fas.usda.gov/esrquery/}

USDA's Export Sales Reporting Program monitors U.S. agricultural export sales on a daily and weekly basis. Export sales reporting provides a constant stream of up-to-date market information for 40 U.S. agricultural commodities sold abroad. The weekly U.S. Export Sales report is the most currently available source of U.S. exports sales data. The data is used to analyze overall levels of export demand, determine where markets exit, and assess the relative position of U.S. commodities in foreign markets.

\section{Global Agricultural Trade System (GATS)}

https://apps.fas.usda.gov/gats/default.aspx

The Global Agricultural Trade System (GATS) is a searchable database containing monthly U.S. Census Bureau trade data organized by agricultural commodity and agricultural related product groups. Trade data is searchable by partner countries and partner groups. Historical U.S. agricultural trade data is available back to 1967. In addition, U.N. trade statistics (UN Comtrade) may be queried through GATS. UN trade data is available for nearly 200 countries or areas, dating from the inception of the Harmonized System (HS) of trade codes in 1989 to present. The database is continuously updated. U.S. trade data is updated monthly according to the U.S. Census Bureau's reporting system. UN Comtrade data are updated in GATS after nationally submitted data to the UN are standardized by the UN Statistical Division and added to the UN Comtrade database.

\section{Other USDA Reports:}

World Agricultural Supply and Demand Estimates (WASDE): http://www.usda.gov/oce/commodity/wasde/

Economic Research Service:

http://www.ers.usda.gov/topics/crops

National Agricultural Statistics Service:

http://www.nass.usda.gov/Publications/ 
Table 01 World Crop Production Summary

Million Metric Tons

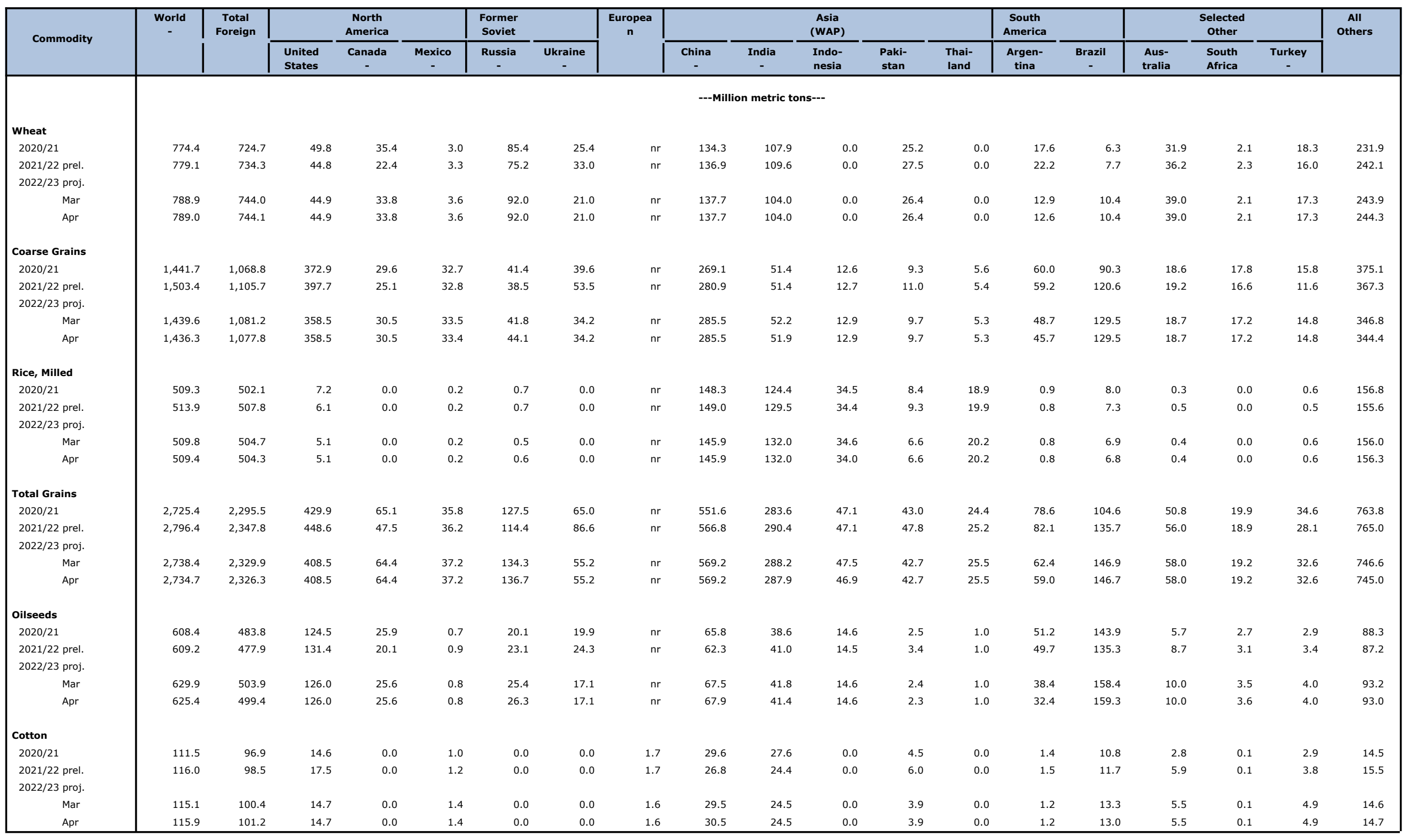

1/ Includes wheat, coarse grains, and rice (milled) shown above. 
Table 02 Wheat Area, Yield, and Production

\begin{tabular}{|c|c|c|c|c|c|c|c|c|c|c|c|c|c|c|c|c|}
\hline \multirow{3}{*}{ Country / Region } & \multicolumn{4}{|c|}{$\begin{array}{c}\text { Area } \\
\text { (Million hectares) }\end{array}$} & \multicolumn{4}{|c|}{$\begin{array}{c}\text { Yield } \\
\text { (Metric tons per hectare) }\end{array}$} & \multicolumn{4}{|c|}{$\begin{array}{c}\text { Production } \\
\text { (Million metric tons) }\end{array}$} & \multicolumn{4}{|c|}{ Change in Production } \\
\hline & \multirow[b]{2}{*}{$2020 / 21$} & \multirow{2}{*}{$\begin{array}{c}\text { Prel. } \\
2021 / 22\end{array}$} & \multicolumn{2}{|c|}{ 2022/23 Proj. } & \multirow[b]{2}{*}{$2020 / 21$} & \multirow{2}{*}{$\begin{array}{c}\text { Prel. } \\
2021 / 22\end{array}$} & \multicolumn{2}{|c|}{ 2022/23 Proj. } & \multirow[b]{2}{*}{$2020 / 21$} & \multirow{2}{*}{$\begin{array}{c}\text { Prel. } \\
2021 / 22\end{array}$} & \multicolumn{2}{|c|}{ 2022/23 Proj. } & \multicolumn{2}{|c|}{ From last month } & \multicolumn{2}{|c|}{ From last year } \\
\hline & & & Mar & Apr & & & Mar & Apr & & & Mar & Apr & MMT & Percent & MMT & Percent \\
\hline World & 220.43 & 221.72 & 220.51 & 220.62 & 3.51 & 3.51 & 3.58 & 3.58 & 774.41 & 779.13 & 788.94 & 789.02 & 0.08 & 0.01 & 9.89 & 1.27 \\
\hline United States & 14.89 & 15.03 & 14.36 & 14.36 & 3.34 & 2.98 & 3.13 & 3.13 & 49.75 & 44.80 & 44.90 & 44.90 & 0.00 & 0.00 & 0.10 & 0.22 \\
\hline Total Foreign & 205.55 & 206.69 & 206.15 & 206.26 & 3.53 & 3.55 & 3.61 & 3.61 & 724.66 & 734.32 & 744.04 & 744.11 & 0.08 & 0.01 & 9.79 & 1.33 \\
\hline European Union & 22.97 & 24.28 & 24.30 & 24.35 & 5.51 & 5.69 & 5.54 & 5.52 & 126.68 & 138.24 & 134.70 & 134.34 & -0.36 & -0.27 & -3.90 & -2.82 \\
\hline United Kingdom & 1.39 & 1.79 & 1.80 & 1.80 & 6.96 & 7.81 & 8.61 & 8.61 & 9.66 & 13.99 & 15.50 & 15.50 & 0.00 & 0.00 & 1.51 & 10.81 \\
\hline Serbia & 0.59 & 0.60 & 0.63 & 0.63 & 4.58 & 5.00 & 4.60 & 4.60 & 2.70 & 3.00 & 2.90 & 2.90 & 0.00 & 0.00 & -0.10 & -3.33 \\
\hline China & 23.38 & 23.57 & 23.52 & 23.52 & 5.74 & 5.81 & 5.86 & 5.86 & 134.25 & 136.95 & 137.72 & 137.72 & 0.00 & 0.00 & 0.78 & 0.57 \\
\hline \multicolumn{17}{|l|}{ South Asia } \\
\hline India & 31.36 & 31.13 & 30.46 & 30.46 & 3.44 & 3.52 & 3.41 & 3.41 & 107.86 & 109.59 & 104.00 & 104.00 & 0.00 & 0.00 & -5.59 & -5.10 \\
\hline Pakistan & 8.81 & 9.17 & 9.00 & 9.00 & 2.87 & 3.00 & 2.93 & 2.93 & 25.25 & 27.46 & 26.40 & 26.40 & 0.00 & 0.00 & -1.06 & -3.87 \\
\hline Afghanistan & 2.33 & 2.20 & 2.20 & 2.20 & 2.15 & 2.00 & 2.05 & 2.05 & 5.01 & 4.40 & 4.50 & 4.50 & 0.00 & 0.00 & 0.10 & 2.27 \\
\hline Nepal & 0.71 & 0.72 & 0.72 & 0.72 & 2.99 & 2.90 & 2.92 & 2.92 & 2.13 & 2.08 & 2.10 & 2.10 & 0.00 & 0.00 & 0.03 & 1.20 \\
\hline \multicolumn{17}{|c|}{ Former Soviet Union - 12} \\
\hline Russia Winter & 16.63 & 15.22 & 16.30 & 16.30 & 3.77 & 3.43 & 4.17 & 4.17 & 62.69 & 52.19 & 68.00 & 68.00 & 0.00 & 0.00 & 15.81 & 30.28 \\
\hline Russia Spring & 12.06 & 12.41 & 12.80 & 12.70 & 1.88 & 1.85 & 1.88 & 1.89 & 22.66 & 22.96 & 24.00 & 24.00 & 0.00 & 0.00 & 1.04 & 4.51 \\
\hline Ukraine & 6.85 & 7.41 & 5.35 & 5.35 & 3.71 & 4.45 & 3.93 & 3.93 & 25.42 & 33.01 & 21.00 & 21.00 & 0.00 & 0.00 & -12.01 & -36.38 \\
\hline Kazakhstan & 12.06 & 12.72 & 12.81 & 12.81 & 1.18 & 0.93 & 1.28 & 1.28 & 14.26 & 11.81 & 16.40 & 16.40 & 0.00 & 0.00 & 4.59 & 38.85 \\
\hline Uzbekistan & 1.36 & 1.36 & 1.40 & 1.40 & 4.53 & 4.40 & 4.42 & 4.42 & 6.16 & 5.99 & 6.19 & 6.19 & 0.00 & 0.00 & 0.21 & 3.44 \\
\hline Belarus & 0.72 & 0.65 & 0.68 & 0.68 & 3.94 & 3.54 & 3.48 & 3.48 & 2.85 & 2.30 & 2.35 & 2.35 & 0.00 & 0.00 & 0.05 & 2.17 \\
\hline Canada & 10.02 & 9.20 & 10.08 & 10.08 & 3.54 & 2.44 & 3.35 & 3.35 & 35.44 & 22.42 & 33.82 & 33.82 & 0.00 & 0.00 & 11.40 & 50.85 \\
\hline \multicolumn{17}{|l|}{ South America } \\
\hline Argentina & 6.40 & 6.55 & 5.50 & 5.50 & 2.76 & 3.38 & 2.35 & 2.28 & 17.64 & 22.15 & 12.90 & 12.55 & -0.35 & -2.71 & -9.60 & -43.34 \\
\hline Brazil & 2.34 & 2.74 & 3.09 & 3.09 & 2.67 & 2.81 & 3.37 & 3.37 & 6.25 & 7.70 & 10.40 & 10.40 & 0.00 & 0.00 & 2.70 & 35.06 \\
\hline Australia & 12.64 & 12.73 & 13.00 & 13.00 & 2.52 & 2.85 & 3.00 & 3.00 & 31.92 & 36.24 & 39.00 & 39.00 & 0.00 & 0.00 & 2.76 & 7.62 \\
\hline \multicolumn{17}{|l|}{ Africa } \\
\hline Egypt & 1.39 & 1.40 & 1.53 & 1.53 & 6.40 & 6.43 & 6.41 & 6.41 & 8.90 & 9.00 & 9.80 & 9.80 & 0.00 & 0.00 & 0.80 & 8.89 \\
\hline Morocco & 2.76 & 2.86 & 2.50 & 2.50 & 0.93 & 2.64 & 1.08 & 1.08 & 2.56 & 7.54 & 2.70 & 2.70 & 0.00 & 0.00 & -4.84 & -64.19 \\
\hline Algeria & 2.08 & 2.08 & 2.08 & 2.08 & 1.50 & 1.20 & 1.78 & 1.78 & 3.11 & 2.50 & 3.70 & 3.70 & 0.00 & 0.00 & 1.20 & 48.00 \\
\hline Ethiopia & 1.83 & 1.95 & 2.00 & 2.30 & 3.00 & 2.83 & 2.85 & 3.04 & 5.48 & 5.52 & 5.70 & 7.00 & 1.30 & 22.81 & 1.48 & 26.81 \\
\hline Iran & 6.00 & 6.00 & 6.20 & 6.20 & 2.50 & 2.00 & 2.13 & 2.13 & 15.00 & 12.00 & 13.20 & 13.20 & 0.00 & 0.00 & 1.20 & 10.00 \\
\hline Iraq & 2.40 & 2.00 & 2.00 & 2.00 & 1.93 & 1.75 & 1.50 & 1.50 & 4.64 & 3.50 & 3.00 & 3.00 & 0.00 & 0.00 & -0.50 & -14.29 \\
\hline Syria & 1.60 & 1.10 & 1.20 & 1.20 & 2.81 & 1.82 & 1.75 & 1.75 & 4.50 & 2.00 & 2.10 & 2.10 & 0.00 & 0.00 & 0.10 & 5.00 \\
\hline Mexico & 0.56 & 0.55 & 0.59 & 0.59 & 5.33 & 6.00 & 6.05 & 6.07 & 2.97 & 3.28 & 3.57 & 3.57 & 0.00 & 0.06 & 0.29 & 8.87 \\
\hline bthers & 7.24 & 7.28 & 7.62 & 7.48 & 2.82 & 2.82 & 2.77 & 2.75 & 20.44 & 20.51 & 21.13 & 20.61 & -0.52 & -2.45 & 0.10 & 0.50 \\
\hline
\end{tabular}

World and Selected Countries and Regions

Foreign Agricultural Service/USDA

April 2023

Global Market Analysis 
Table 03 Total Coarse Grain Area, Yield, and Production

\begin{tabular}{|c|c|c|c|c|c|c|c|c|c|c|c|c|c|c|c|c|}
\hline \multirow{3}{*}{ Country / Region } & \multicolumn{4}{|c|}{$\begin{array}{c}\text { Area } \\
\text { (Million hectares) }\end{array}$} & \multicolumn{4}{|c|}{$\begin{array}{c}\text { Yield } \\
\text { (Metric tons per hectare) }\end{array}$} & \multicolumn{4}{|c|}{$\begin{array}{c}\text { Production } \\
\text { (Million metric tons) }\end{array}$} & \multicolumn{4}{|c|}{ Change in Production } \\
\hline & \multirow[b]{2}{*}{$2020 / 21$} & Prel. & \multicolumn{2}{|c|}{ 2022/23 Proj. } & \multirow[b]{2}{*}{$2020 / 21$} & \multirow{2}{*}{$\begin{array}{c}\text { Prel. } \\
2021 / 22\end{array}$} & \multicolumn{2}{|c|}{ 2022/23 Proj. } & \multirow[b]{2}{*}{$2020 / 21$} & \multirow{2}{*}{$\begin{array}{c}\text { Prel. } \\
\text { 2021/22 }\end{array}$} & \multicolumn{2}{|c|}{ 2022/23 Proj. } & \multicolumn{2}{|c|}{ From last month } & \multicolumn{2}{|c|}{ From last year } \\
\hline & & $2021 / 22$ & Mar & Apr & & & Mar & Apr & & & Mar & Apr & MMT & Percent & MMT & Percent \\
\hline World & 344.41 & 345.07 & 337.50 & 336.33 & 4.19 & 4.36 & 4.27 & 4.27 & $1,441.67$ & $1,503.40$ & $1,439.62$ & $1,436.29$ & -3.33 & -0.23 & -67.11 & -4.46 \\
\hline United States & 36.81 & 38.34 & 35.39 & 35.39 & 10.13 & 10.37 & 10.13 & 10.13 & 372.89 & 397.71 & 358.47 & 358.47 & 0.00 & 0.00 & -39.24 & -9.87 \\
\hline Total Foreign & 307.60 & 306.73 & 302.11 & 300.95 & 3.47 & 3.60 & 3.58 & 3.58 & $1,068.78$ & $1,105.69$ & $1,081.15$ & $1,077.82$ & -3.33 & -0.31 & -27.86 & -2.52 \\
\hline China & 43.72 & 45.77 & 45.52 & 45.52 & 6.15 & 6.14 & 6.27 & 6.27 & 269.08 & 280.85 & 285.50 & 285.50 & 0.00 & 0.00 & 4.65 & 1.65 \\
\hline European Union & 28.77 & 27.74 & 27.23 & 26.76 & 5.40 & 5.58 & 4.98 & 5.00 & 155.46 & 154.77 & 135.61 & 133.85 & -1.76 & -1.30 & -20.92 & -13.52 \\
\hline United Kingdom & 1.66 & 1.43 & 1.37 & 1.37 & 5.64 & 5.88 & 6.32 & 6.32 & 9.36 & 8.41 & 8.65 & 8.65 & 0.00 & 0.00 & 0.24 & 2.84 \\
\hline \multicolumn{17}{|l|}{ South America } \\
\hline Brazil & 21.30 & 23.37 & 24.31 & 24.31 & 4.24 & 5.16 & 5.33 & 5.33 & 90.32 & 120.62 & 129.54 & 129.54 & 0.00 & 0.00 & 8.92 & 7.39 \\
\hline Argentina & 8.65 & 9.84 & 9.30 & 9.49 & 6.94 & 6.01 & 5.24 & 4.82 & 60.01 & 59.16 & 48.71 & 45.71 & -3.00 & -6.16 & -13.45 & -22.73 \\
\hline \multicolumn{17}{|l|}{ Former Soviet Union - 12} \\
\hline Russia & 14.55 & 14.01 & 13.50 & 13.60 & 2.84 & 2.75 & 3.09 & 3.25 & 41.40 & 38.54 & 41.77 & 44.13 & 2.36 & 5.65 & 5.59 & 14.52 \\
\hline Ukraine & 8.53 & 8.66 & 6.37 & 6.37 & 4.64 & 6.18 & 5.37 & 5.37 & 39.59 & 53.51 & 34.16 & 34.16 & 0.00 & 0.00 & -19.35 & -36.17 \\
\hline Kazakhstan & 3.20 & 2.63 & 2.63 & 2.63 & 1.54 & 1.43 & 1.79 & 1.79 & 4.93 & 3.76 & 4.71 & 4.71 & 0.00 & 0.00 & 0.96 & 25.46 \\
\hline Belarus & 1.14 & 0.99 & 1.03 & 1.03 & 3.47 & 3.05 & 3.11 & 3.11 & 3.95 & 3.00 & 3.20 & 3.20 & 0.00 & 0.00 & 0.20 & 6.67 \\
\hline \multicolumn{17}{|l|}{ Africa } \\
\hline Nigeria & 13.10 & 13.70 & 13.50 & 13.50 & 1.60 & 1.56 & 1.57 & 1.57 & 20.90 & 21.39 & 21.18 & 21.18 & 0.00 & 0.00 & -0.21 & -0.99 \\
\hline South Africa & 3.36 & 3.19 & 3.18 & 3.18 & 5.31 & 5.22 & 5.39 & 5.39 & 17.81 & 16.64 & 17.16 & 17.16 & 0.00 & 0.00 & 0.52 & 3.13 \\
\hline Tanzania & 5.17 & 5.40 & 5.00 & 5.00 & 1.51 & 1.40 & 1.40 & 1.40 & 7.79 & 7.55 & 6.98 & 6.98 & 0.00 & 0.00 & -0.58 & -7.62 \\
\hline Burkina Faso & 3.89 & 4.24 & 4.24 & 4.24 & 1.21 & 0.99 & 1.16 & 1.16 & 4.72 & 4.20 & 4.90 & 4.90 & 0.00 & 0.00 & 0.70 & 16.58 \\
\hline Ethiopia & 5.53 & 5.59 & 5.63 & 5.63 & 3.45 & 3.08 & 3.23 & 3.23 & 19.10 & 17.20 & 18.20 & 18.20 & 0.00 & 0.00 & 1.00 & 5.81 \\
\hline Egypt & 1.03 & 1.16 & 1.16 & 1.16 & 7.01 & 7.13 & 7.13 & 7.13 & 7.24 & 8.30 & 8.30 & 8.30 & 0.00 & 0.00 & 0.00 & 0.00 \\
\hline Mali & 5.17 & 4.70 & 4.70 & 4.70 & 1.41 & 1.34 & 1.45 & 1.45 & 7.26 & 6.31 & 6.80 & 6.80 & 0.00 & 0.00 & 0.49 & 7.70 \\
\hline India & 24.12 & 23.35 & 24.55 & 23.80 & 2.13 & 2.20 & 2.13 & 2.18 & 51.39 & 51.39 & 52.21 & 51.91 & -0.30 & -0.57 & 0.52 & 1.02 \\
\hline \multicolumn{17}{|l|}{ Southeast Asia } \\
\hline Indonesia & 3.85 & 3.90 & 3.95 & 3.95 & 3.27 & 3.26 & 3.27 & 3.27 & 12.60 & 12.70 & 12.90 & 12.90 & 0.00 & 0.00 & 0.20 & 1.57 \\
\hline Philippines & 2.57 & 2.56 & 2.50 & 2.50 & 3.25 & 3.26 & 3.24 & 3.24 & 8.35 & 8.34 & 8.10 & 8.10 & 0.00 & 0.00 & -0.24 & -2.92 \\
\hline Vietnam & 0.94 & 0.90 & 0.89 & 0.87 & 4.83 & 4.92 & 4.94 & 4.97 & 4.56 & 4.45 & 4.40 & 4.30 & -0.10 & -2.27 & -0.15 & -3.28 \\
\hline Thailand & 1.28 & 1.23 & 1.23 & 1.21 & 4.33 & 4.36 & 4.28 & 4.35 & 5.55 & 5.35 & 5.25 & 5.25 & 0.00 & 0.00 & -0.10 & -1.87 \\
\hline Mexico & 8.76 & 8.88 & 8.99 & 8.97 & 3.73 & 3.69 & 3.73 & 3.73 & 32.66 & 32.79 & 33.49 & 33.44 & -0.05 & -0.15 & 0.65 & 1.98 \\
\hline Canada & 5.80 & 5.91 & 5.74 & 5.74 & 5.11 & 4.25 & 5.32 & 5.32 & 29.64 & 25.12 & 30.54 & 30.54 & 0.00 & 0.00 & 5.41 & 21.54 \\
\hline Australia & 7.26 & 6.69 & 5.74 & 5.74 & 2.56 & 2.87 & 3.25 & 3.25 & 18.56 & 19.22 & 18.67 & 18.67 & 0.00 & 0.00 & -0.55 & -2.86 \\
\hline \multicolumn{17}{|l|}{ Middle East } \\
\hline Turkey & 4.63 & 4.46 & 4.55 & 4.55 & 3.40 & 2.60 & 3.25 & 3.25 & 15.76 & 11.58 & 14.77 & 14.77 & 0.00 & 0.00 & 3.19 & 27.55 \\
\hline Iran & 1.81 & 1.91 & 1.86 & 1.86 & 2.77 & 2.16 & 2.38 & 2.38 & 5.02 & 4.12 & 4.42 & 4.42 & 0.00 & 0.00 & 0.30 & 7.31 \\
\hline bthers & 77.82 & 74.51 & 73.47 & 73.30 & 1.62 & 1.70 & 1.65 & 1.65 & 125.79 & 126.43 & 121.06 & 120.58 & -0.48 & -0.40 & -5.85 & -4.63 \\
\hline
\end{tabular}

World and Selected Countries and Regions; Coarse Grain includes: Barley, Corn, Millet, Mixed Grains, Oats, Rye and Sorghum 
Table 04 Corn Area, Yield, and Production

\begin{tabular}{|c|c|c|c|c|c|c|c|c|c|c|c|c|c|c|c|c|}
\hline \multirow{3}{*}{ Country / Region } & \multicolumn{4}{|c|}{$\begin{array}{c}\text { Area } \\
\text { (Million hectares) } \\
\end{array}$} & \multicolumn{4}{|c|}{$\begin{array}{c}\text { Yield } \\
\text { (Metric tons per hectare) } \\
\end{array}$} & \multicolumn{4}{|c|}{$\begin{array}{c}\text { Production } \\
\text { (Million metric tons) }\end{array}$} & \multicolumn{4}{|c|}{ Change in Production } \\
\hline & \multirow[b]{2}{*}{$2020 / 21$} & Prel. & \multicolumn{2}{|c|}{ 2022/23 Proj. } & \multirow[b]{2}{*}{$2020 / 21$} & \multirow{2}{*}{$\begin{array}{c}\text { Prel. } \\
\text { 2021/22 }\end{array}$} & \multicolumn{2}{|c|}{ 2022/23 Proj. } & \multirow[b]{2}{*}{$2020 / 21$} & \multirow{2}{*}{$\begin{array}{c}\text { Prel. } \\
\text { 2021/22 }\end{array}$} & \multicolumn{2}{|c|}{ 2022/23 Proj. } & \multicolumn{2}{|c|}{ From last month } & \multicolumn{2}{|c|}{ From last year } \\
\hline & & $2021 / 22$ & Mar & Apr & & & Mar & Apr & & & Mar & Apr & MMT & Percent & MMT & Percent \\
\hline World & 199.09 & 206.89 & 201.29 & 201.16 & 5.67 & 5.88 & 5.70 & 5.69 & $1,129.42$ & $1,217.00$ & $1,147.52$ & $1,144.50$ & -3.02 & -0.26 & -72.50 & -5.96 \\
\hline United States & 33.31 & 34.53 & 32.05 & 32.05 & 10.76 & 11.09 & 10.88 & 10.88 & 358.45 & 382.89 & 348.75 & 348.75 & 0.00 & 0.00 & -34.14 & -8.92 \\
\hline Total Foreign & 165.78 & 172.37 & 169.23 & 169.11 & 4.65 & 4.84 & 4.72 & 4.71 & 770.98 & 834.11 & 798.77 & 795.75 & -3.02 & -0.38 & -38.36 & -4.60 \\
\hline China & 41.26 & 43.32 & 43.07 & 43.07 & 6.32 & 6.29 & 6.44 & 6.44 & 260.67 & 272.55 & 277.20 & 277.20 & 0.00 & 0.00 & 4.65 & 1.71 \\
\hline \multicolumn{17}{|l|}{ South America } \\
\hline Brazil & 19.90 & 21.80 & 22.70 & 22.70 & 4.37 & 5.32 & 5.51 & 5.51 & 87.00 & 116.00 & 125.00 & 125.00 & 0.00 & 0.00 & 9.00 & 7.76 \\
\hline Argentina & 6.55 & 7.10 & 6.70 & 6.70 & 7.94 & 6.97 & 5.97 & 5.52 & 52.00 & 49.50 & 40.00 & 37.00 & -3.00 & -7.50 & -12.50 & -25.25 \\
\hline Bolivia & 0.41 & 0.44 & 0.44 & 0.44 & 3.00 & 2.60 & 2.64 & 2.64 & 1.23 & 1.13 & 1.15 & 1.15 & 0.00 & 0.00 & 0.02 & 1.77 \\
\hline European Union & 9.24 & 9.23 & 9.03 & 8.87 & 7.30 & 7.73 & 6.01 & 5.97 & 67.44 & 71.37 & 54.20 & 52.97 & -1.23 & -2.27 & -18.40 & -25.78 \\
\hline \multicolumn{17}{|l|}{ Africa } \\
\hline South Africa & 3.12 & 3.00 & 3.00 & 3.00 & 5.44 & 5.38 & 5.57 & 5.57 & 16.95 & 16.14 & 16.70 & 16.70 & 0.00 & 0.00 & 0.56 & 3.49 \\
\hline Nigeria & 5.50 & 6.00 & 5.80 & 5.80 & 2.25 & 2.12 & 2.10 & 2.10 & 12.40 & 12.75 & 12.18 & 12.18 & 0.00 & 0.00 & -0.57 & -4.43 \\
\hline Ethiopia & 2.36 & 2.53 & 2.55 & 2.55 & 4.47 & 3.72 & 4.00 & 4.00 & 10.56 & 9.40 & 10.20 & 10.20 & 0.00 & 0.00 & 0.80 & 8.51 \\
\hline Egypt & 0.80 & 0.93 & 0.93 & 0.93 & 8.00 & 8.00 & 8.00 & 8.00 & 6.40 & 7.44 & 7.44 & 7.44 & 0.00 & 0.00 & 0.00 & 0.00 \\
\hline Tanzania & 4.20 & 4.40 & 4.00 & 4.00 & 1.60 & 1.48 & 1.48 & 1.48 & 6.71 & 6.50 & 5.90 & 5.90 & 0.00 & 0.00 & -0.60 & -9.23 \\
\hline Malawi & 1.76 & 1.75 & 1.75 & 1.75 & 2.10 & 2.62 & 2.12 & 2.12 & 3.69 & 4.58 & 3.72 & 3.72 & 0.00 & 0.00 & -0.86 & -18.86 \\
\hline Zambia & 1.33 & 1.41 & 1.12 & 1.12 & 2.54 & 2.57 & 2.43 & 2.43 & 3.39 & 3.62 & 2.71 & 2.71 & 0.00 & 0.00 & -0.91 & -25.25 \\
\hline Kenya & 2.19 & 1.95 & 1.90 & 1.90 & 1.73 & 1.59 & 1.58 & 1.58 & 3.79 & 3.10 & 3.00 & 3.00 & 0.00 & 0.00 & -0.10 & -3.23 \\
\hline Uganda & 0.99 & 1.10 & 1.10 & 1.10 & 2.78 & 2.55 & 2.55 & 2.55 & 2.75 & 2.80 & 2.80 & 2.80 & 0.00 & 0.00 & 0.00 & 0.00 \\
\hline Zimbabwe & 1.58 & 1.95 & 1.90 & 1.90 & 0.57 & 1.39 & 0.82 & 0.82 & 0.91 & 2.72 & 1.56 & 1.56 & 0.00 & 0.00 & -1.16 & -42.66 \\
\hline \multicolumn{17}{|l|}{ Former Soviet Union - 12} \\
\hline Ukraine & 5.40 & 5.49 & 4.00 & 4.00 & 5.62 & 7.68 & 6.75 & 6.75 & 30.30 & 42.13 & 27.00 & 27.00 & 0.00 & 0.00 & -15.13 & -35.91 \\
\hline Russia & 2.73 & 2.90 & 2.50 & 2.64 & 5.08 & 5.25 & 5.60 & 6.00 & 13.87 & 15.23 & 14.00 & 15.83 & 1.83 & 13.09 & 0.61 & 3.99 \\
\hline \multicolumn{17}{|l|}{ South Asia } \\
\hline India & 9.89 & 9.96 & 10.10 & 10.10 & 3.20 & 3.39 & 3.43 & 3.43 & 31.65 & 33.73 & 34.61 & 34.61 & 0.00 & 0.00 & 0.88 & 2.61 \\
\hline Pakistan & 1.42 & 1.65 & 1.60 & 1.60 & 6.30 & 6.43 & 5.75 & 5.75 & 8.94 & 10.64 & 9.20 & 9.20 & 0.00 & 0.00 & -1.44 & -13.49 \\
\hline Nepal & 0.97 & 0.97 & 0.97 & 0.97 & 2.82 & 2.82 & 2.82 & 2.82 & 2.72 & 2.72 & 2.72 & 2.72 & 0.00 & 0.00 & 0.00 & 0.00 \\
\hline \multicolumn{17}{|l|}{ Southeast Asia } \\
\hline Indonesia & 3.85 & 3.90 & 3.95 & 3.95 & 3.27 & 3.26 & 3.27 & 3.27 & 12.60 & 12.70 & 12.90 & 12.90 & 0.00 & 0.00 & 0.20 & 1.57 \\
\hline Philippines & 2.57 & 2.56 & 2.50 & 2.50 & 3.25 & 3.26 & 3.24 & 3.24 & 8.35 & 8.34 & 8.10 & 8.10 & 0.00 & 0.00 & -0.24 & -2.92 \\
\hline Vietnam & 0.94 & 0.90 & 0.89 & 0.87 & 4.83 & 4.92 & 4.94 & 4.97 & 4.56 & 4.45 & 4.40 & 4.30 & -0.10 & -2.27 & -0.15 & -3.28 \\
\hline Thailand & 1.26 & 1.20 & 1.20 & 1.18 & 4.38 & 4.42 & 4.33 & 4.41 & 5.50 & 5.30 & 5.20 & 5.20 & 0.00 & 0.00 & -0.10 & -1.89 \\
\hline Mexico & 7.14 & 7.09 & 7.20 & 7.20 & 3.83 & 3.77 & 3.83 & 3.83 & 27.35 & 26.76 & 27.60 & 27.60 & 0.00 & 0.00 & 0.84 & 3.13 \\
\hline Canada & 1.41 & 1.46 & 1.44 & 1.44 & 9.63 & 9.99 & 10.07 & 10.07 & 13.56 & 14.61 & 14.54 & 14.54 & 0.00 & 0.00 & -0.07 & -0.49 \\
\hline Turkey & 0.62 & 0.54 & 0.53 & 0.53 & 11.45 & 12.04 & 12.83 & 12.83 & 7.10 & 6.50 & 6.80 & 6.80 & 0.00 & 0.00 & 0.30 & 4.62 \\
\hline bthers & 26.38 & 26.83 & 26.38 & 26.31 & 2.60 & 2.66 & 2.58 & 2.56 & 68.60 & 71.42 & 67.95 & 67.43 & -0.52 & -0.77 & -3.99 & -5.59 \\
\hline
\end{tabular}

World and Selected Countries and Regions 
Table 05 Barley Area, Yield, and Production

\begin{tabular}{|c|c|c|c|c|c|c|c|c|c|c|c|c|c|c|c|c|}
\hline \multirow{3}{*}{ Country / Region } & \multicolumn{4}{|c|}{$\begin{array}{c}\text { Area } \\
\text { (Million hectares) }\end{array}$} & \multicolumn{4}{|c|}{$\begin{array}{c}\text { Yield } \\
\text { (Metric tons per hectare) }\end{array}$} & \multicolumn{4}{|c|}{$\begin{array}{c}\text { Production } \\
\text { (Million metric tons) }\end{array}$} & \multicolumn{4}{|c|}{ Change in Production } \\
\hline & \multirow[b]{2}{*}{$2020 / 21$} & Prel. & \multicolumn{2}{|c|}{ 2022/23 Proj. } & \multirow[b]{2}{*}{$2020 / 21$} & \multirow{2}{*}{$\begin{array}{c}\text { Prel. } \\
2021 / 22 \\
\end{array}$} & \multicolumn{2}{|c|}{ 2022/23 Proj. } & \multirow[b]{2}{*}{$2020 / 21$} & \multirow{2}{*}{$\begin{array}{c}\text { Prel. } \\
2021 / 22\end{array}$} & \multicolumn{2}{|c|}{ 2022/23 Proj. } & \multicolumn{2}{|c|}{ From last month } & \multicolumn{2}{|c|}{ From last year } \\
\hline & & $2021 / 22$ & Mar & Apr & & & Mar & Apr & & & Mar & Apr & MMT & Percent & MMT & Percent \\
\hline United States & 0.90 & 0.81 & 0.99 & 0.99 & 4.15 & 3.25 & 3.85 & 3.85 & 3.72 & 2.62 & 3.80 & 3.80 & 0.00 & 0.00 & 1.18 & 45.16 \\
\hline Total Foreign & 51.55 & 48.49 & 46.28 & 46.38 & 3.05 & 2.96 & 3.19 & 3.19 & 157.20 & 143.35 & 147.83 & 148.16 & 0.33 & 0.23 & 4.81 & 3.36 \\
\hline European Union & 11.05 & 10.30 & 10.43 & 10.42 & 4.91 & 5.05 & 4.93 & 4.97 & 54.23 & 52.05 & 51.45 & 51.80 & 0.35 & 0.68 & -0.25 & -0.48 \\
\hline United Kingdom & 1.39 & 1.15 & 1.12 & 1.12 & 5.85 & 6.05 & 6.64 & 6.64 & 8.12 & 6.96 & 7.40 & 7.40 & 0.00 & 0.00 & 0.44 & 6.31 \\
\hline \multicolumn{17}{|l|}{ Former Soviet Union - 12} \\
\hline Russia & 8.16 & 7.69 & 7.80 & 7.75 & 2.53 & 2.28 & 2.76 & 2.77 & 20.63 & 17.51 & 21.50 & 21.50 & 0.00 & 0.00 & 4.00 & 22.82 \\
\hline Ukraine & 2.58 & 2.68 & 1.90 & 1.90 & 3.08 & 3.70 & 3.21 & 3.21 & 7.95 & 9.92 & 6.10 & 6.10 & 0.00 & 0.00 & -3.82 & -38.53 \\
\hline Kazakhstan & 2.73 & 2.16 & 2.18 & 2.18 & 1.34 & 1.10 & 1.51 & 1.51 & 3.66 & 2.37 & 3.29 & 3.29 & 0.00 & 0.00 & 0.92 & 38.87 \\
\hline Belarus & 0.41 & 0.35 & 0.40 & 0.40 & 3.35 & 2.86 & 2.75 & 2.75 & 1.38 & 1.00 & 1.10 & 1.10 & 0.00 & 0.00 & 0.10 & 10.00 \\
\hline Azerbaijan & 0.35 & 0.37 & 0.38 & 0.38 & 2.96 & 3.07 & 2.93 & 2.93 & 1.02 & 1.15 & 1.10 & 1.10 & 0.00 & 0.00 & -0.05 & -4.10 \\
\hline Canada & 2.81 & 3.01 & 2.64 & 2.64 & 3.82 & 2.32 & 3.79 & 3.79 & 10.74 & 6.98 & 9.99 & 9.99 & 0.00 & 0.00 & 3.00 & 43.00 \\
\hline Turkey & 3.80 & 3.70 & 3.80 & 3.80 & 2.13 & 1.22 & 1.95 & 1.95 & 8.10 & 4.50 & 7.40 & 7.40 & 0.00 & 0.00 & 2.90 & 64.44 \\
\hline Iran & 1.60 & 1.70 & 1.65 & 1.65 & 2.25 & 1.59 & 1.82 & 1.82 & 3.60 & 2.70 & 3.00 & 3.00 & 0.00 & 0.00 & 0.30 & 11.11 \\
\hline Iraq & 1.20 & 0.60 & 0.60 & 0.60 & 1.29 & 1.17 & 1.13 & 1.13 & 1.55 & 0.70 & 0.68 & 0.68 & 0.00 & 0.00 & -0.02 & -2.86 \\
\hline Syria & 1.50 & 0.80 & 0.90 & 0.90 & 1.27 & 0.81 & 0.78 & 0.78 & 1.90 & 0.65 & 0.70 & 0.70 & 0.00 & 0.00 & 0.05 & 7.69 \\
\hline \multicolumn{17}{|l|}{ Africa } \\
\hline Ethiopia & 0.90 & 0.96 & 0.97 & 0.97 & 2.52 & 2.45 & 2.47 & 2.47 & 2.26 & 2.35 & 2.40 & 2.40 & 0.00 & 0.00 & 0.05 & 2.13 \\
\hline Morocco & 1.47 & 1.49 & 1.10 & 1.10 & 0.44 & 1.87 & 0.64 & 0.64 & 0.64 & 2.78 & 0.70 & 0.70 & 0.00 & 0.00 & -2.08 & -74.82 \\
\hline Algeria & 0.98 & 1.03 & 1.03 & 1.03 & 1.24 & 0.93 & 1.56 & 1.56 & 1.21 & 0.95 & 1.60 & 1.60 & 0.00 & 0.00 & 0.65 & 68.42 \\
\hline Tunisia & 0.36 & 0.28 & 0.30 & 0.30 & 1.28 & 1.54 & 1.40 & 1.53 & 0.47 & 0.43 & 0.42 & 0.46 & 0.04 & 9.52 & 0.03 & 6.98 \\
\hline South Africa & 0.14 & 0.10 & 0.10 & 0.10 & 4.14 & 3.52 & 3.06 & 3.06 & 0.59 & 0.33 & 0.31 & 0.31 & 0.00 & 0.00 & -0.03 & -7.49 \\
\hline \multicolumn{17}{|l|}{ South America } \\
\hline Argentina & 1.01 & 1.34 & 1.38 & 1.57 & 4.00 & 3.96 & 3.27 & 2.88 & 4.04 & 5.30 & 4.50 & 4.50 & 0.00 & 0.00 & -0.80 & -15.09 \\
\hline Uruguay & 0.19 & 0.22 & 0.21 & 0.21 & 4.78 & 4.11 & 4.25 & 4.25 & 0.89 & 0.92 & 0.90 & 0.90 & 0.00 & 0.00 & -0.02 & -2.50 \\
\hline Brazil & 0.10 & 0.11 & 0.11 & 0.11 & 3.63 & 3.79 & 3.91 & 3.91 & 0.37 & 0.43 & 0.43 & 0.43 & 0.00 & 0.00 & 0.01 & 1.18 \\
\hline India & 0.59 & 0.59 & 0.45 & 0.45 & 2.92 & 2.80 & 3.02 & 3.02 & 1.72 & 1.66 & 1.37 & 1.37 & 0.00 & 0.00 & -0.29 & -17.27 \\
\hline China & 0.51 & 0.51 & 0.51 & 0.51 & 4.00 & 3.92 & 3.92 & 3.92 & 2.04 & 2.00 & 2.00 & 2.00 & 0.00 & 0.00 & 0.00 & 0.00 \\
\hline pthers & 1.77 & 1.75 & 1.75 & 1.74 & 2.43 & 2.34 & 2.41 & 2.42 & 4.31 & 4.09 & 4.22 & 4.21 & -0.01 & -0.12 & 0.12 & 3.03 \\
\hline
\end{tabular}

World and Selected Countries and Regions 
Table 06 Oats Area, Yield, and Production

\begin{tabular}{|c|c|c|c|c|c|c|c|c|c|c|c|c|c|c|c|c|}
\hline \multirow{3}{*}{ Country / Region } & \multicolumn{4}{|c|}{$\begin{array}{c}\text { Area } \\
\text { (Million hectares) } \\
\end{array}$} & \multicolumn{4}{|c|}{$\begin{array}{c}\text { Yield } \\
\text { (Metric tons per hectare) } \\
\end{array}$} & \multicolumn{4}{|c|}{$\begin{array}{c}\text { Production } \\
\text { (Million metric tons) } \\
\end{array}$} & \multicolumn{4}{|c|}{ Change in Production } \\
\hline & \multirow[b]{2}{*}{$2020 / 21$} & Prel. & \multicolumn{2}{|c|}{ 2022/23 Proj. } & \multirow[b]{2}{*}{$2020 / 21$} & \multirow{2}{*}{$\begin{array}{c}\text { Prel. } \\
2021 / 22\end{array}$} & \multicolumn{2}{|c|}{ 2022/23 Proj. } & \multirow[b]{2}{*}{$2020 / 21$} & \multirow{2}{*}{$\begin{array}{c}\text { Prel. } \\
2021 / 22\end{array}$} & \multicolumn{2}{|c|}{ 2022/23 Proj. } & \multicolumn{2}{|c|}{ From last month } & \multicolumn{2}{|c|}{ From last year } \\
\hline & & $2021 / 22$ & Mar & Apr & & & Mar & Apr & & & Mar & Apr & MMT & Percent & MMT & Percent \\
\hline World & 10.08 & 9.63 & 9.55 & 9.55 & 2.55 & 2.36 & 2.65 & 2.70 & 25.72 & 22.72 & 25.25 & 25.77 & 0.52 & 2.07 & 3.05 & 13.42 \\
\hline United States & 0.41 & 0.26 & 0.36 & 0.36 & 2.34 & 2.20 & 2.33 & 2.33 & 0.95 & 0.58 & 0.84 & 0.84 & 0.00 & 0.00 & 0.26 & 44.81 \\
\hline Total Foreign & 9.67 & 9.37 & 9.19 & 9.19 & 2.56 & 2.36 & 2.66 & 2.71 & 24.77 & 22.15 & 24.42 & 24.94 & 0.52 & 2.14 & 2.79 & 12.60 \\
\hline European Union & 2.56 & 2.54 & 2.40 & 2.37 & 3.31 & 2.95 & 3.18 & 3.22 & 8.47 & 7.52 & 7.61 & 7.63 & 0.02 & 0.30 & 0.12 & 1.53 \\
\hline \multicolumn{17}{|l|}{ Former Soviet Union - 12} \\
\hline Russia & 2.33 & 2.17 & 2.10 & 2.13 & 1.77 & 1.72 & 1.90 & 2.11 & 4.13 & 3.73 & 4.00 & 4.50 & 0.50 & 12.50 & 0.77 & 20.55 \\
\hline Ukraine & 0.20 & 0.18 & 0.18 & 0.18 & 2.54 & 2.61 & 2.29 & 2.29 & 0.52 & 0.48 & 0.40 & 0.40 & 0.00 & 0.00 & -0.08 & -16.32 \\
\hline Belarus & 0.16 & 0.15 & 0.16 & 0.16 & 2.87 & 2.33 & 2.42 & 2.42 & 0.45 & 0.35 & 0.38 & 0.38 & 0.00 & 0.00 & 0.03 & 7.14 \\
\hline Kazakhstan & 0.23 & 0.20 & 0.20 & 0.20 & 1.05 & 0.90 & 1.16 & 1.16 & 0.24 & 0.18 & 0.23 & 0.23 & 0.00 & 0.00 & 0.05 & 25.82 \\
\hline Canada & 1.31 & 1.21 & 1.40 & 1.40 & 3.48 & 2.39 & 3.73 & 3.73 & 4.58 & 2.90 & 5.23 & 5.23 & 0.00 & 0.00 & 2.33 & 80.27 \\
\hline \multicolumn{17}{|l|}{ South America } \\
\hline Argentina & 0.24 & 0.35 & 0.28 & 0.28 & 2.13 & 2.07 & 2.30 & 2.30 & 0.51 & 0.73 & 0.65 & 0.65 & 0.00 & 0.00 & -0.08 & -11.03 \\
\hline Brazil & 0.43 & 0.50 & 0.51 & 0.51 & 2.00 & 2.27 & 2.26 & 2.26 & 0.85 & 1.14 & 1.16 & 1.16 & 0.00 & 0.00 & 0.01 & 1.05 \\
\hline Chile & 0.11 & 0.12 & 0.13 & 0.13 & 4.65 & 4.70 & 4.37 & 4.37 & 0.53 & 0.58 & 0.55 & 0.55 & 0.00 & 0.00 & -0.03 & -4.84 \\
\hline Uruguay & 0.02 & 0.01 & 0.02 & 0.02 & 2.20 & 2.21 & 2.40 & 2.40 & 0.03 & 0.03 & 0.04 & 0.04 & 0.00 & 0.00 & 0.01 & 16.13 \\
\hline \multicolumn{17}{|l|}{ Pceania } \\
\hline Australia & 1.07 & 0.84 & 0.80 & 0.80 & 1.77 & 2.06 & 2.00 & 2.00 & 1.90 & 1.74 & 1.60 & 1.60 & 0.00 & 0.00 & -0.14 & -7.78 \\
\hline New Zealand & 0.00 & 0.00 & 0.01 & 0.01 & 5.75 & 6.25 & 5.67 & 5.67 & 0.02 & 0.03 & 0.03 & 0.03 & 0.00 & 0.00 & 0.01 & 36.00 \\
\hline China & 0.41 & 0.41 & 0.41 & 0.41 & 1.48 & 1.48 & 1.48 & 1.48 & 0.60 & 0.60 & 0.60 & 0.60 & 0.00 & 0.00 & 0.00 & 0.00 \\
\hline \multicolumn{17}{|l|}{ Africa } \\
\hline Algeria & 0.06 & 0.08 & 0.08 & 0.08 & 1.11 & 1.31 & 1.31 & 1.31 & 0.07 & 0.11 & 0.11 & 0.11 & 0.00 & 0.00 & 0.00 & 0.00 \\
\hline Morocco & 0.02 & 0.04 & 0.02 & 0.02 & 0.40 & 1.13 & 0.40 & 0.40 & 0.01 & 0.05 & 0.01 & 0.01 & 0.00 & 0.00 & -0.04 & -86.67 \\
\hline South Africa & 0.03 & 0.04 & 0.03 & 0.03 & 2.19 & 1.64 & 1.11 & 1.11 & 0.06 & 0.06 & 0.03 & 0.03 & 0.00 & 0.00 & -0.03 & -49.15 \\
\hline \multicolumn{17}{|l|}{ pther Europe } \\
\hline United Kingdom & 0.21 & 0.20 & 0.19 & 0.19 & 4.91 & 5.62 & 5.26 & 5.26 & 1.03 & 1.12 & 1.00 & 1.00 & 0.00 & 0.00 & -0.12 & -10.95 \\
\hline Norway & 0.07 & 0.07 & 0.07 & 0.07 & 4.29 & 4.29 & 4.29 & 4.29 & 0.30 & 0.30 & 0.30 & 0.30 & 0.00 & 0.00 & 0.00 & 0.00 \\
\hline Serbia & 0.03 & 0.03 & 0.03 & 0.03 & 2.17 & 2.17 & 2.17 & 2.17 & 0.07 & 0.07 & 0.07 & 0.07 & 0.00 & 0.00 & 0.00 & 0.00 \\
\hline Albania & 0.02 & 0.02 & 0.02 & 0.02 & 2.25 & 2.20 & 2.20 & 2.20 & 0.04 & 0.03 & 0.03 & 0.03 & 0.00 & 0.00 & 0.00 & 0.00 \\
\hline Bosnia and Herzegovina & 0.01 & 0.01 & 0.01 & 0.01 & 3.09 & 2.82 & 2.82 & 2.82 & 0.03 & 0.03 & 0.03 & 0.03 & 0.00 & 0.00 & 0.00 & 0.00 \\
\hline Turkey & 0.10 & 0.11 & 0.11 & 0.11 & 2.40 & 2.38 & 2.38 & 2.38 & 0.24 & 0.25 & 0.25 & 0.25 & 0.00 & 0.00 & 0.00 & 0.00 \\
\hline Mexico & 0.03 & 0.05 & 0.05 & 0.05 & 2.03 & 2.02 & 1.89 & 1.89 & 0.06 & 0.09 & 0.09 & 0.09 & 0.00 & 0.00 & -0.01 & -8.60 \\
\hline pthers & 0.03 & 0.03 & 0.03 & 0.03 & 1.58 & 1.62 & 1.68 & 1.65 & 0.05 & 0.05 & 0.05 & 0.05 & 0.00 & -1.92 & 0.00 & 8.51 \\
\hline
\end{tabular}

World and Selected Countries and Regions 
Table 07 Rye Area, Yield, and Production

\begin{tabular}{|c|c|c|c|c|c|c|c|c|c|c|c|c|c|c|c|c|}
\hline \multirow{3}{*}{ Country / Region } & \multicolumn{4}{|c|}{$\begin{array}{c}\text { Area } \\
\text { (Million hectares) }\end{array}$} & \multicolumn{4}{|c|}{$\begin{array}{c}\text { Yield } \\
\text { (Metric tons per hectare) }\end{array}$} & \multicolumn{4}{|c|}{$\begin{array}{c}\text { Production } \\
\text { (Million metric tons) }\end{array}$} & \multicolumn{4}{|c|}{ Change in Production } \\
\hline & \multirow[b]{2}{*}{$2020 / 21$} & Prel. & \multicolumn{2}{|c|}{ 2022/23 Proj. } & \multirow[b]{2}{*}{$2020 / 21$} & \multirow{2}{*}{$\begin{array}{c}\text { Prel. } \\
2021 / 22\end{array}$} & \multicolumn{2}{|c|}{ 2022/23 Proj. } & \multirow[b]{2}{*}{$2020 / 21$} & \multirow{2}{*}{$\begin{array}{c}\text { Prel. } \\
2021 / 22\end{array}$} & \multicolumn{2}{|c|}{ 2022/23 Proj. } & \multicolumn{2}{|c|}{ From last month } & \multicolumn{2}{|c|}{ From last year } \\
\hline & & $2021 / 22$ & Mar & Apr & & & Mar & Apr & & & Mar & Apr & MMT & Percent & MMT & Percent \\
\hline World & 4.17 & 4.03 & 3.79 & 3.66 & 3.43 & 3.10 & 3.21 & 3.31 & 14.30 & 12.50 & 12.19 & 12.11 & -0.08 & -0.65 & -0.40 & -3.17 \\
\hline United States & 0.13 & 0.12 & 0.14 & 0.14 & 2.19 & 2.09 & 2.26 & 2.26 & 0.29 & 0.25 & 0.31 & 0.31 & 0.00 & 0.00 & 0.06 & 25.30 \\
\hline Total Foreign & 4.03 & 3.91 & 3.65 & 3.52 & 3.47 & 3.13 & 3.25 & 3.35 & 14.01 & 12.25 & 11.87 & 11.80 & -0.08 & -0.67 & -0.46 & -3.75 \\
\hline European Union & 2.08 & 1.93 & 1.87 & 1.76 & 4.31 & 4.15 & 4.07 & 4.28 & 8.97 & 8.01 & 7.62 & 7.54 & -0.08 & -1.04 & -0.47 & -5.89 \\
\hline \multicolumn{17}{|l|}{ Former Soviet Union - 12} \\
\hline Russia & 0.97 & 0.99 & 0.90 & 0.88 & 2.44 & 1.73 & 2.22 & 2.27 & 2.38 & 1.72 & 2.00 & 2.00 & 0.00 & 0.00 & 0.28 & 16.55 \\
\hline Belarus & 0.36 & 0.33 & 0.30 & 0.30 & 2.92 & 2.46 & 2.50 & 2.50 & 1.05 & 0.80 & 0.75 & 0.75 & 0.00 & 0.00 & -0.05 & -6.25 \\
\hline Ukraine & 0.14 & 0.18 & 0.10 & 0.10 & 3.28 & 3.43 & 2.85 & 2.85 & 0.46 & 0.60 & 0.29 & 0.29 & 0.00 & 0.00 & -0.32 & -52.50 \\
\hline Kazakhstan & 0.02 & 0.04 & 0.03 & 0.03 & 1.25 & 0.91 & 1.76 & 1.76 & 0.03 & 0.04 & 0.06 & 0.06 & 0.00 & 0.00 & 0.02 & 50.00 \\
\hline Turkey & 0.11 & 0.12 & 0.11 & 0.11 & 2.91 & 2.87 & 2.91 & 2.91 & 0.32 & 0.33 & 0.32 & 0.32 & 0.00 & 0.00 & -0.01 & -3.03 \\
\hline Canada & 0.15 & 0.12 & 0.15 & 0.15 & 3.19 & 3.21 & 3.42 & 3.42 & 0.49 & 0.37 & 0.52 & 0.52 & 0.00 & 0.00 & 0.15 & 39.78 \\
\hline \multicolumn{17}{|l|}{ South America } \\
\hline \multicolumn{17}{|l|}{ bther Europe } \\
\hline Bosnia and Herzegovina & 0.00 & 0.00 & 0.00 & 0.00 & 3.67 & 3.00 & 2.75 & 2.75 & 0.01 & 0.01 & 0.01 & 0.01 & 0.00 & 0.00 & 0.00 & 22.22 \\
\hline Switzerland & 0.00 & 0.00 & 0.00 & 0.00 & 5.50 & 5.50 & 5.50 & 5.50 & 0.01 & 0.01 & 0.01 & 0.01 & 0.00 & 0.00 & 0.00 & 0.00 \\
\hline Serbia & 0.00 & 0.00 & 0.00 & 0.00 & 2.50 & 2.50 & 2.50 & 2.50 & 0.01 & 0.01 & 0.01 & 0.01 & 0.00 & 0.00 & 0.00 & 0.00 \\
\hline Australia & 0.05 & 0.05 & 0.05 & 0.05 & 0.71 & 0.71 & 0.71 & 0.71 & 0.03 & 0.03 & 0.03 & 0.03 & 0.00 & 0.00 & 0.00 & 0.00 \\
\hline pthers & 0.04 & 0.04 & 0.04 & 0.04 & 2.50 & 2.18 & 2.16 & 2.16 & 0.11 & 0.10 & 0.10 & 0.10 & 0.00 & 0.00 & 0.00 & -1.04 \\
\hline
\end{tabular}

World and Selected Countries and Regions 
Table 08 Sorghum Area, Yield, and Production

\begin{tabular}{|c|c|c|c|c|c|c|c|c|c|c|c|c|c|c|c|c|}
\hline \multirow{3}{*}{ Country / Region } & \multicolumn{4}{|c|}{$\begin{array}{c}\text { Area } \\
\text { (Million hectares) }\end{array}$} & \multicolumn{4}{|c|}{$\begin{array}{c}\text { Yield } \\
\text { (Metric tons per hectare) }\end{array}$} & \multicolumn{4}{|c|}{$\begin{array}{c}\text { Production } \\
\text { (Million metric tons) }\end{array}$} & \multicolumn{4}{|c|}{ Change in Production } \\
\hline & \multirow[b]{2}{*}{$2020 / 21$} & Prel. & \multicolumn{2}{|c|}{ 2022/23 Proj. } & \multirow[b]{2}{*}{$2020 / 21$} & \multirow{2}{*}{$\begin{array}{c}\text { Prel. } \\
2021 / 22\end{array}$} & \multicolumn{2}{|c|}{ 2022/23 Proj. } & \multirow[b]{2}{*}{$2020 / 21$} & \multirow{2}{*}{$\begin{array}{c}\text { Prel. } \\
2021 / 22\end{array}$} & \multicolumn{2}{|c|}{ 2022/23 Proj. } & \multicolumn{2}{|c|}{ From last month } & \multicolumn{2}{|c|}{ From last year } \\
\hline & & $2021 / 22$ & Mar & Apr & & & Mar & Apr & & & Mar & Apr & MMT & Percent & MMT & Percent \\
\hline World & 42.73 & 40.53 & 40.77 & 39.91 & 1.47 & 1.53 & 1.42 & 1.45 & 62.79 & 61.95 & 58.03 & 57.69 & -0.34 & -0.59 & -4.27 & -6.88 \\
\hline United States & 2.06 & 2.63 & 1.85 & 1.85 & 4.59 & 4.33 & 2.58 & 2.58 & 9.47 & 11.38 & 4.77 & 4.77 & 0.00 & 0.00 & -6.61 & -58.07 \\
\hline Total Foreign & 40.67 & 37.90 & 38.92 & 38.06 & 1.31 & 1.33 & 1.37 & 1.39 & 53.32 & 50.58 & 53.26 & 52.92 & -0.34 & -0.64 & 2.34 & 4.63 \\
\hline \multicolumn{17}{|l|}{ Africa } \\
\hline Nigeria & 5.60 & 5.70 & 5.70 & 5.70 & 1.18 & 1.18 & 1.23 & 1.23 & 6.59 & 6.73 & 7.00 & 7.00 & 0.00 & 0.00 & 0.28 & 4.09 \\
\hline Ethiopia & 1.79 & 1.65 & 1.66 & 1.66 & 2.83 & 2.70 & 2.71 & 2.71 & 5.06 & 4.45 & 4.50 & 4.50 & 0.00 & 0.00 & 0.05 & 1.12 \\
\hline Sudan & 9.03 & 6.92 & 7.00 & 7.00 & 0.57 & 0.51 & 0.71 & 0.71 & 5.15 & 3.53 & 5.00 & 5.00 & 0.00 & 0.00 & 1.47 & 41.64 \\
\hline Burkina Faso & 1.74 & 1.90 & 1.90 & 1.90 & 1.06 & 0.87 & 1.00 & 1.00 & 1.84 & 1.64 & 1.90 & 1.90 & 0.00 & 0.00 & 0.26 & 15.57 \\
\hline Mali & 1.83 & 1.50 & 1.50 & 1.50 & 1.00 & 0.83 & 1.00 & 1.00 & 1.82 & 1.24 & 1.50 & 1.50 & 0.00 & 0.00 & 0.26 & 21.07 \\
\hline Niger & 3.67 & 3.70 & 3.70 & 3.70 & 0.58 & 0.33 & 0.51 & 0.51 & 2.13 & 1.21 & 1.90 & 1.90 & 0.00 & 0.00 & 0.69 & 57.42 \\
\hline Cameroon & 0.74 & 0.74 & 0.74 & 0.74 & 1.62 & 1.62 & 1.62 & 1.62 & 1.20 & 1.20 & 1.20 & 1.20 & 0.00 & 0.00 & 0.00 & 0.00 \\
\hline Tanzania & 0.70 & 0.70 & 0.70 & 0.70 & 1.07 & 1.02 & 1.07 & 1.07 & 0.75 & 0.72 & 0.75 & 0.75 & 0.00 & 0.00 & 0.04 & 4.90 \\
\hline Egypt & 0.15 & 0.15 & 0.15 & 0.15 & 4.91 & 5.00 & 5.00 & 5.00 & 0.73 & 0.75 & 0.75 & 0.75 & 0.00 & 0.00 & 0.00 & 0.00 \\
\hline Uganda & 0.31 & 0.25 & 0.25 & 0.25 & 1.23 & 1.32 & 0.90 & 0.90 & 0.38 & 0.33 & 0.23 & 0.23 & 0.00 & 0.00 & -0.11 & -31.82 \\
\hline Ghana & 0.30 & 0.31 & 0.31 & 0.31 & 1.13 & 1.11 & 1.13 & 1.13 & 0.35 & 0.35 & 0.35 & 0.35 & 0.00 & 0.00 & 0.01 & 1.45 \\
\hline Mozambique & 0.26 & 0.25 & 0.25 & 0.25 & 0.54 & 0.64 & 0.64 & 0.64 & 0.14 & 0.16 & 0.16 & 0.16 & 0.00 & 0.00 & 0.00 & 0.00 \\
\hline South Africa & 0.05 & 0.04 & 0.04 & 0.04 & 4.39 & 2.78 & 3.29 & 3.29 & 0.22 & 0.10 & 0.12 & 0.12 & 0.00 & 0.00 & 0.01 & 11.65 \\
\hline Mexico & 1.29 & 1.40 & 1.42 & 1.42 & 3.37 & 3.47 & 3.42 & 3.42 & 4.35 & 4.84 & 4.85 & 4.85 & 0.00 & 0.00 & 0.01 & 0.21 \\
\hline \multicolumn{17}{|l|}{ South America } \\
\hline Argentina & 0.75 & 0.93 & 0.85 & 0.85 & 4.43 & 3.68 & 4.00 & 4.00 & 3.32 & 3.40 & 3.40 & 3.40 & 0.00 & 0.00 & 0.00 & 0.00 \\
\hline Brazil & 0.87 & 0.95 & 0.98 & 0.98 & 2.41 & 3.20 & 3.00 & 3.00 & 2.08 & 3.04 & 2.94 & 2.94 & 0.00 & 0.00 & -0.10 & -3.35 \\
\hline \multicolumn{17}{|l|}{ South Asia } \\
\hline India & 4.38 & 3.80 & 4.50 & 3.75 & 1.10 & 1.09 & 0.98 & 1.09 & 4.81 & 4.15 & 4.40 & 4.10 & -0.30 & -6.82 & -0.05 & -1.20 \\
\hline Pakistan & 0.13 & 0.08 & 0.22 & 0.22 & 0.76 & 0.83 & 0.63 & 0.63 & 0.10 & 0.06 & 0.14 & 0.14 & 0.00 & 0.00 & 0.07 & 110.94 \\
\hline China & 0.64 & 0.63 & 0.63 & 0.63 & 4.68 & 4.76 & 4.76 & 4.76 & 2.97 & 3.00 & 3.00 & 3.00 & 0.00 & 0.00 & 0.00 & 0.00 \\
\hline Australia & 0.57 & 0.62 & 0.70 & 0.70 & 2.86 & 4.26 & 3.57 & 3.57 & 1.64 & 2.65 & 2.50 & 2.50 & 0.00 & 0.00 & -0.15 & -5.59 \\
\hline European Union & 0.19 & 0.15 & 0.13 & 0.13 & 5.21 & 5.41 & 4.41 & 4.27 & 1.00 & 0.80 & 0.59 & 0.55 & -0.04 & -6.77 & -0.25 & -31.21 \\
\hline pthers & 5.69 & 5.55 & 5.60 & 5.49 & 1.18 & 1.12 & 1.09 & 1.11 & 6.70 & 6.23 & 6.09 & 6.09 & 0.00 & 0.00 & -0.14 & -2.29 \\
\hline
\end{tabular}

World and Selected Countries and Regions 
Table 09 Rice Area, Yield, and Production

World and Selected Countries and Regions

\begin{tabular}{|c|c|c|c|c|c|c|c|c|c|c|c|c|c|c|c|c|}
\hline \multirow{3}{*}{ Country / Region } & \multicolumn{4}{|c|}{$\begin{array}{c}\text { Area } \\
\text { (Million hectares) } \\
\end{array}$} & \multicolumn{4}{|c|}{$\begin{array}{c}\text { Yield } \\
\text { (Metric tons per hectare) }\end{array}$} & \multicolumn{4}{|c|}{$\begin{array}{c}\text { Production } \\
\text { (Million metric tons) } \\
\end{array}$} & \multicolumn{4}{|c|}{ Change in Production } \\
\hline & \multirow[b]{2}{*}{$2020 / 21$} & \multirow{2}{*}{$\begin{array}{l}\text { Prel. } \\
2021 / 22\end{array}$} & \multicolumn{2}{|c|}{ 2022/23 Proj. } & \multirow[b]{2}{*}{$2020 / 21$} & \multirow{2}{*}{$\begin{array}{c}\text { Prel. } \\
2021 / 22\end{array}$} & \multicolumn{2}{|c|}{ 2022/23 Proj. } & \multirow[b]{2}{*}{$2020 / 21$} & \multirow{2}{*}{$\begin{array}{c}\text { Prel. } \\
2021 / 22\end{array}$} & \multicolumn{2}{|c|}{ 2022/23 Proj. } & \multicolumn{2}{|c|}{ From last month } & \multicolumn{2}{|c|}{ From last year } \\
\hline & & & Mar & Apr & & & Mar & Apr & & & Mar & Apr & MMT & Percent & MMT & Percent \\
\hline United States & 1.21 & 1.01 & 0.88 & 0.88 & 8.54 & 8.64 & 8.28 & 8.28 & 7.22 & 6.08 & 5.09 & 5.09 & 0.00 & 0.00 & -0.99 & -16.29 \\
\hline Total Foreign & 164.00 & 164.90 & 164.32 & 164.24 & 4.57 & 4.60 & 4.59 & 4.59 & 502.10 & 507.77 & 504.74 & 504.33 & -0.41 & -0.08 & -3.44 & -0.68 \\
\hline \multicolumn{17}{|l|}{ East Asia } \\
\hline China & 30.08 & 29.92 & 29.45 & 29.45 & 7.04 & 7.11 & 7.08 & 7.08 & 148.30 & 148.99 & 145.95 & 145.95 & 0.00 & 0.00 & -3.04 & -2.04 \\
\hline Japan & 1.53 & 1.52 & 1.50 & 1.50 & 6.78 & 6.90 & 6.82 & 6.86 & 7.57 & 7.64 & 7.45 & 7.48 & 0.03 & 0.40 & -0.16 & -2.04 \\
\hline Korea, South & 0.73 & 0.73 & 0.73 & 0.73 & 6.49 & 7.12 & 6.88 & 6.88 & 3.51 & 3.88 & 3.76 & 3.76 & 0.00 & 0.00 & -0.12 & -3.04 \\
\hline Korea, North & 0.49 & 0.50 & 0.50 & 0.50 & 4.44 & 4.18 & 4.18 & 4.18 & 1.40 & 1.36 & 1.36 & 1.36 & 0.00 & 0.00 & 0.00 & 0.00 \\
\hline \multicolumn{17}{|l|}{ South Asia } \\
\hline India & 45.77 & 46.28 & 47.00 & 47.00 & 4.08 & 4.20 & 4.21 & 4.21 & 124.37 & 129.47 & 132.00 & 132.00 & 0.00 & 0.00 & 2.53 & 1.95 \\
\hline Bangladesh & 11.50 & 11.62 & 11.50 & 11.60 & 4.51 & 4.63 & 4.68 & 4.70 & 34.60 & 35.85 & 35.85 & 36.35 & 0.50 & 1.39 & 0.50 & 1.39 \\
\hline Pakistan & 3.34 & 3.54 & 3.00 & 3.00 & 3.79 & 3.95 & 3.30 & 3.30 & 8.42 & 9.32 & 6.60 & 6.60 & 0.00 & 0.00 & -2.72 & -29.21 \\
\hline Sri Lanka & 1.09 & 1.13 & 1.09 & 1.09 & 4.59 & 3.56 & 3.41 & 3.41 & 3.39 & 2.73 & 2.53 & 2.53 & 0.00 & 0.00 & -0.21 & -7.61 \\
\hline \multicolumn{17}{|l|}{ Southeast Asia } \\
\hline Indonesia & 11.40 & 11.60 & 11.65 & 11.55 & 4.77 & 4.67 & 4.68 & 4.64 & 34.50 & 34.40 & 34.60 & 34.00 & -0.60 & -1.73 & -0.40 & -1.16 \\
\hline Vietnam & 7.31 & 7.19 & 7.17 & 7.17 & 6.00 & 5.96 & 6.03 & 6.03 & 27.38 & 26.77 & 27.00 & 27.00 & 0.00 & 0.00 & 0.23 & 0.86 \\
\hline Thailand & 10.51 & 10.70 & 10.85 & 10.85 & 2.72 & 2.81 & 2.82 & 2.82 & 18.86 & 19.88 & 20.20 & 20.20 & 0.00 & 0.00 & 0.32 & 1.62 \\
\hline Burma & 6.90 & 6.90 & 6.90 & 6.90 & 2.85 & 2.80 & 2.83 & 2.83 & 12.60 & 12.35 & 12.50 & 12.50 & 0.00 & 0.00 & 0.15 & 1.20 \\
\hline Philippines & 4.76 & 4.80 & 4.80 & 4.80 & 4.14 & 4.15 & 4.10 & 4.10 & 12.42 & 12.54 & 12.41 & 12.41 & 0.00 & 0.00 & -0.13 & -1.03 \\
\hline Cambodia & 3.26 & 3.27 & 3.31 & 3.31 & 2.89 & 2.89 & 2.94 & 2.94 & 5.74 & 5.77 & 5.93 & 5.93 & 0.00 & 0.00 & 0.16 & 2.81 \\
\hline Laos & 0.93 & 0.94 & 0.95 & 0.95 & 3.24 & 3.28 & 3.26 & 3.26 & 1.90 & 1.95 & 1.95 & 1.95 & 0.00 & 0.00 & 0.00 & 0.00 \\
\hline Malaysia & 0.68 & 0.65 & 0.68 & 0.66 & 4.07 & 3.97 & 4.07 & 3.99 & 1.80 & 1.68 & 1.80 & 1.70 & -0.10 & -5.56 & 0.02 & 1.37 \\
\hline \multicolumn{17}{|l|}{ South America } \\
\hline Brazil & 1.68 & 1.62 & 1.47 & 1.47 & 7.00 & 6.67 & 6.94 & 6.80 & 8.00 & 7.34 & 6.94 & 6.80 & -0.14 & -1.96 & -0.54 & -7.32 \\
\hline Peru & 0.41 & 0.42 & 0.42 & 0.42 & 8.22 & 8.37 & 8.55 & 8.55 & 2.34 & 2.43 & 2.48 & 2.48 & 0.00 & 0.00 & 0.05 & 2.22 \\
\hline \multicolumn{17}{|l|}{ Africa } \\
\hline Egypt & 0.70 & 0.50 & 0.60 & 0.60 & 8.28 & 8.41 & 8.70 & 8.70 & 4.00 & 2.90 & 3.60 & 3.60 & 0.00 & 0.00 & 0.70 & 24.14 \\
\hline Madagascar & 1.68 & 1.65 & 1.60 & 1.60 & 2.53 & 2.65 & 2.69 & 2.69 & 2.71 & 2.80 & 2.75 & 2.75 & 0.00 & 0.00 & -0.05 & -1.82 \\
\hline Nigeria & 3.60 & 3.65 & 3.50 & 3.50 & 2.27 & 2.29 & 2.29 & 2.29 & 5.15 & 5.26 & 5.04 & 5.04 & 0.00 & 0.00 & -0.22 & -4.09 \\
\hline pthers & 13.21 & 13.33 & 13.31 & 13.26 & 2.94 & 2.89 & 2.86 & 2.86 & 25.58 & 25.41 & 25.11 & 24.94 & -0.17 & -0.67 & -0.47 & -1.85 \\
\hline
\end{tabular}

Yield is on a rough basis, before the milling process. Production is on a milled basis, after the milling process. 
Table 10 Total Oilseed Area, Yield, and Production

World and Selected Countries and Regions

\begin{tabular}{|c|c|c|c|c|c|c|c|c|c|c|c|c|c|c|c|c|}
\hline \multirow{3}{*}{ Country / Region } & \multicolumn{4}{|c|}{$\begin{array}{c}\text { Area } \\
\text { (Million hectares) }\end{array}$} & \multicolumn{4}{|c|}{$\begin{array}{c}\text { Yield } \\
\text { (Metric tons per hectare) }\end{array}$} & \multicolumn{4}{|c|}{$\begin{array}{c}\text { Production } \\
\text { (Million metric tons) }\end{array}$} & \multicolumn{4}{|c|}{ Change in Production } \\
\hline & \multirow[b]{2}{*}{$2020 / 21$} & Prel. & \multicolumn{2}{|c|}{ 2022/23 Proj. } & \multirow[b]{2}{*}{$2020 / 21$} & Prel. & \multicolumn{2}{|c|}{ 2022/23 Proj. } & \multirow[b]{2}{*}{$2020 / 21$} & \multirow{2}{*}{ Prel. } & \multicolumn{2}{|c|}{ 2022/23 Proj. } & \multicolumn{2}{|c|}{ From last month } & \multicolumn{2}{|c|}{ From last year } \\
\hline & & $2021 / 22$ & Mar & Apr & & $2021 / 22$ & Mar & Apr & & & Mar & Apr & MMT & Percent & MMT & Percent \\
\hline World Total & -- & $\overline{--}$ & -- & -- & -- & $\overline{--}$ & -- & -- & 608.35 & 609.21 & 629.89 & 625.38 & -4.51 & -0.72 & 16.17 & 2.65 \\
\hline Total Foreign & -- & -- & -- & -- & -- & -- & -- & -- & 483.83 & 477.86 & 503.93 & 499.41 & -4.51 & -0.90 & 21.55 & 4.51 \\
\hline Dilseed, Copra & -- & -- & -- & -- & -- & -- & -- & -- & 5.78 & 6.07 & 6.03 & 6.03 & 0.00 & 0.00 & -0.04 & -0.61 \\
\hline bilseed, Palm Kernel & -- & -- & -- & -- & -- & -- & -- & -- & 19.09 & 19.33 & 20.08 & 20.08 & 0.00 & 0.00 & 0.74 & 3.84 \\
\hline Major OilSeeds & 252.13 & 261.07 & 263.85 & 263.73 & 2.31 & 2.24 & 2.29 & 2.27 & 583.49 & 583.81 & 603.79 & 599.27 & -4.51 & -0.75 & 15.46 & 2.65 \\
\hline United States & 38.81 & 41.06 & 40.04 & 40.04 & 3.21 & 3.20 & 3.15 & 3.15 & 124.52 & 131.35 & 125.96 & 125.96 & 0.00 & 0.00 & -5.39 & -4.10 \\
\hline Foreign Oilseeds & 213.32 & 220.00 & 223.81 & 223.69 & 2.15 & 2.06 & 2.13 & 2.12 & 458.97 & 452.46 & 477.82 & 473.31 & -4.51 & -0.94 & 20.85 & 4.61 \\
\hline South America & 66.35 & 68.92 & 70.59 & 70.16 & 3.18 & 2.86 & 3.02 & 2.95 & 210.89 & 196.92 & 213.23 & 207.24 & -5.99 & -2.81 & 10.33 & 5.24 \\
\hline Brazil & 41.07 & 43.43 & 45.28 & 45.58 & 3.50 & 3.11 & 3.49 & 3.49 & 143.77 & 135.17 & 158.23 & 159.13 & 0.90 & 0.57 & 23.96 & 17.73 \\
\hline Argentina & 18.97 & 18.77 & 18.51 & 18.01 & 2.70 & 2.65 & 2.08 & 1.80 & 51.21 & 49.67 & 38.44 & 32.42 & -6.02 & -15.66 & -17.25 & -34.73 \\
\hline Paraguay & 3.38 & 3.51 & 3.54 & 3.56 & 2.89 & 1.23 & 2.86 & 2.85 & 9.77 & 4.31 & 10.13 & 10.17 & 0.04 & 0.41 & 5.86 & 135.84 \\
\hline China & 25.45 & 24.10 & 26.14 & 26.14 & 2.59 & 2.59 & 2.58 & 2.60 & 65.81 & 62.32 & 67.49 & 67.88 & 0.39 & 0.58 & 5.56 & 8.93 \\
\hline South Asia & 42.50 & 41.80 & 42.92 & 42.53 & 0.97 & 1.06 & 1.02 & 1.03 & 41.05 & 44.34 & 43.64 & 44.02 & 0.38 & 0.88 & -0.32 & -0.73 \\
\hline India & 39.15 & 38.48 & 40.18 & 39.18 & 0.96 & 1.04 & 1.01 & 1.03 & 37.56 & 39.94 & 40.77 & 40.42 & -0.35 & -0.86 & 0.48 & 1.20 \\
\hline Pakistan & 2.65 & 2.59 & 2.38 & 2.43 & 0.94 & 1.31 & 1.02 & 0.96 & 2.50 & 3.38 & 2.43 & 2.34 & -0.09 & -3.58 & -1.03 & -30.59 \\
\hline European Union & 11.10 & 11.06 & 12.09 & 12.51 & 2.59 & 2.80 & 2.64 & 2.54 & 28.77 & 30.98 & 31.94 & 31.79 & -0.16 & -0.49 & 0.81 & 2.61 \\
\hline United Kingdom & 0.38 & 0.31 & 0.36 & 0.36 & 2.73 & 3.20 & 3.40 & 3.40 & 1.04 & 0.98 & 1.23 & 1.23 & 0.00 & 0.00 & 0.24 & 24.87 \\
\hline Former Soviet Union - 12 & 25.91 & 27.74 & 26.09 & 26.32 & 1.73 & 1.89 & 1.82 & 1.84 & 44.71 & 52.45 & 47.58 & 48.43 & 0.85 & 1.79 & -4.03 & -7.68 \\
\hline Russia & 12.50 & 14.23 & 14.50 & 14.74 & 1.61 & 1.62 & 1.75 & 1.78 & 20.14 & 23.11 & 25.40 & 26.25 & 0.85 & 3.35 & 3.14 & 13.60 \\
\hline Ukraine & 9.65 & 9.58 & 7.45 & 7.45 & 2.06 & 2.54 & 2.30 & 2.30 & 19.85 & 24.32 & 17.10 & 17.10 & 0.00 & 0.00 & -7.22 & -29.67 \\
\hline Uzbekistan & 1.08 & 1.08 & 1.09 & 1.09 & 1.20 & 1.10 & 1.09 & 1.09 & 1.29 & 1.19 & 1.19 & 1.19 & 0.00 & 0.00 & 0.00 & 0.08 \\
\hline Canada & 10.41 & 11.06 & 10.76 & 10.76 & 2.49 & 1.81 & 2.38 & 2.38 & 25.95 & 20.05 & 25.63 & 25.63 & 0.00 & 0.00 & 5.58 & 27.81 \\
\hline Africa & 22.12 & 24.79 & 23.83 & 23.85 & 1.08 & 0.98 & 1.05 & 1.06 & 23.99 & 24.36 & 25.08 & 25.20 & 0.12 & 0.48 & 0.84 & 3.44 \\
\hline Nigeria & 4.62 & 4.97 & 4.87 & 4.87 & 1.19 & 1.11 & 1.21 & 1.21 & 5.49 & 5.50 & 5.90 & 5.90 & 0.00 & 0.00 & 0.41 & 7.37 \\
\hline South Africa & 1.36 & 1.66 & 1.76 & 1.76 & 1.98 & 1.89 & 2.00 & 2.05 & 2.69 & 3.14 & 3.52 & 3.61 & 0.09 & 2.41 & 0.47 & 14.84 \\
\hline Tanzania & 1.17 & 1.24 & 1.35 & 1.35 & 1.02 & 0.98 & 0.93 & 0.93 & 1.19 & 1.21 & 1.25 & 1.25 & 0.00 & 0.00 & 0.04 & 3.39 \\
\hline Southeast Asia & 3.25 & 3.21 & 3.18 & 3.18 & 1.36 & 1.36 & 1.38 & 1.38 & 4.42 & 4.38 & 4.37 & 4.37 & 0.00 & 0.00 & -0.01 & -0.16 \\
\hline Australia & 2.94 & 3.94 & 4.64 & 4.64 & 1.93 & 2.20 & 2.17 & 2.17 & 5.69 & 8.68 & 10.05 & 10.05 & 0.00 & 0.00 & 1.37 & 15.83 \\
\hline Turkey & 1.18 & 1.32 & 1.48 & 1.48 & 2.46 & 2.55 & 2.69 & 2.69 & 2.89 & 3.37 & 3.98 & 3.98 & 0.00 & 0.00 & 0.61 & 18.02 \\
\hline pthers & 1.71 & 1.76 & 1.74 & 1.77 & 2.20 & 2.06 & 2.07 & 1.97 & 3.77 & 3.63 & 3.61 & 3.50 & -0.12 & -3.18 & -0.13 & -3.61 \\
\hline
\end{tabular}

World Total and Total Foreign: (Major Oilseeds plus copra and palm kernel) Major Oilseeds: (soybeans, sunflowerseeds, peanuts(inshell), cottonseed and rapeseed) 
Table 11 Soybean Area, Yield, and Production

\begin{tabular}{|c|c|c|c|c|c|c|c|c|c|c|c|c|c|c|c|c|}
\hline \multirow{3}{*}{ Country / Region } & \multicolumn{4}{|c|}{$\begin{array}{c}\text { Area } \\
\text { (Million hectares) }\end{array}$} & \multicolumn{4}{|c|}{$\begin{array}{c}\text { Yield } \\
\text { (Metric tons per hectare) }\end{array}$} & \multicolumn{4}{|c|}{$\begin{array}{c}\text { Production } \\
\text { (Million metric tons) }\end{array}$} & \multicolumn{4}{|c|}{ Change in Production } \\
\hline & \multirow[b]{2}{*}{$2020 / 21$} & Prel. & \multicolumn{2}{|c|}{ 2022/23 Proj. } & \multirow[b]{2}{*}{$2020 / 21$} & \multirow{2}{*}{$\begin{array}{c}\text { Prel. } \\
2021 / 22\end{array}$} & \multicolumn{2}{|c|}{ 2022/23 Proj. } & \multirow[b]{2}{*}{$2020 / 21$} & \multirow{2}{*}{$\begin{array}{c}\text { Prel. } \\
2021 / 22\end{array}$} & \multicolumn{2}{|c|}{ 2022/23 Proj. } & \multicolumn{2}{|c|}{ From last month } & \multicolumn{2}{|c|}{ From last year } \\
\hline & & $2021 / 22$ & Mar & Apr & & & Mar & Apr & & & Mar & Apr & MMT & Percent & MMT & Percent \\
\hline World & 129.27 & 130.98 & 135.24 & 134.18 & 2.85 & 2.75 & 2.77 & 2.75 & 368.60 & 359.80 & 375.15 & 369.64 & -5.51 & -1.47 & 9.84 & 2.74 \\
\hline United States & 33.43 & 34.93 & 34.94 & 34.94 & 3.43 & 3.48 & 3.33 & 3.33 & 114.75 & 121.53 & 116.38 & 116.38 & 0.00 & 0.00 & -5.15 & -4.24 \\
\hline Total Foreign & 95.84 & 96.05 & 100.30 & 99.25 & 2.65 & 2.48 & 2.58 & 2.55 & 253.85 & 238.27 & 258.77 & 253.26 & -5.51 & -2.13 & 14.99 & 6.29 \\
\hline \multicolumn{17}{|l|}{ South America } \\
\hline Brazil & 39.50 & 41.60 & 43.40 & 43.70 & 3.53 & 3.14 & 3.53 & 3.52 & 139.50 & 130.50 & 153.00 & 154.00 & 1.00 & 0.65 & 23.50 & 18.01 \\
\hline Argentina & 16.47 & 15.90 & 15.50 & 15.00 & 2.81 & 2.76 & 2.13 & 1.80 & 46.20 & 43.90 & 33.00 & 27.00 & -6.00 & -18.18 & -16.90 & -38.50 \\
\hline Paraguay & 3.29 & 3.42 & 3.45 & 3.45 & 2.93 & 1.22 & 2.90 & 2.90 & 9.64 & 4.18 & 10.00 & 10.00 & 0.00 & 0.00 & 5.82 & 139.06 \\
\hline Bolivia & 1.43 & 1.55 & 1.43 & 1.43 & 2.32 & 2.32 & 2.17 & 2.17 & 3.32 & 3.60 & 3.10 & 3.10 & 0.00 & 0.00 & -0.50 & -13.89 \\
\hline Uruguay & 1.06 & 1.16 & 1.15 & 0.90 & 1.88 & 2.80 & 1.83 & 1.33 & 2.00 & 3.23 & 2.10 & 1.20 & -0.90 & -42.86 & -2.03 & -62.88 \\
\hline \multicolumn{17}{|l|}{ East Asia } \\
\hline China & 9.88 & 8.42 & 10.24 & 10.24 & 1.98 & 1.95 & 1.98 & 1.98 & 19.60 & 16.40 & 20.28 & 20.28 & 0.00 & 0.00 & 3.89 & 23.70 \\
\hline Korea, South & 0.06 & 0.05 & 0.06 & 0.06 & 1.47 & 2.06 & 1.73 & 1.73 & 0.08 & 0.11 & 0.10 & 0.10 & 0.00 & 0.00 & -0.02 & -14.41 \\
\hline Korea, North & 0.16 & 0.17 & 0.16 & 0.16 & 1.40 & 1.15 & 1.15 & 1.15 & 0.23 & 0.19 & 0.18 & 0.18 & 0.00 & 0.00 & -0.01 & -5.26 \\
\hline Japan & 0.14 & 0.15 & 0.15 & 0.15 & 1.54 & 1.63 & 1.55 & 1.55 & 0.22 & 0.24 & 0.23 & 0.23 & 0.00 & 0.00 & -0.01 & -2.52 \\
\hline India & 12.92 & 12.15 & 12.70 & 12.00 & 0.81 & 0.98 & 0.94 & 1.00 & 10.46 & 11.89 & 12.00 & 12.00 & 0.00 & 0.00 & 0.11 & 0.93 \\
\hline Canada & 2.04 & 2.08 & 2.12 & 2.12 & 3.12 & 2.99 & 3.09 & 3.09 & 6.36 & 6.22 & 6.54 & 6.54 & 0.00 & 0.00 & 0.32 & 5.13 \\
\hline \multicolumn{17}{|l|}{ Former Soviet Union - 12} \\
\hline Russia & 2.71 & 2.99 & 3.30 & 3.36 & 1.59 & 1.59 & 1.67 & 1.79 & 4.31 & 4.76 & 5.50 & 6.00 & 0.50 & 9.02 & 1.24 & 25.97 \\
\hline Ukraine & 1.46 & 1.44 & 1.35 & 1.35 & 2.05 & 2.64 & 2.37 & 2.37 & 3.00 & 3.80 & 3.20 & 3.20 & 0.00 & 0.00 & -0.60 & -15.79 \\
\hline European Union & 1.00 & 0.98 & 1.06 & 1.08 & 2.61 & 2.83 & 2.34 & 2.26 & 2.60 & 2.77 & 2.47 & 2.44 & -0.03 & -1.05 & -0.33 & -11.98 \\
\hline \multicolumn{17}{|l|}{ Southeast Asia } \\
\hline Indonesia & 0.39 & 0.35 & 0.33 & 0.33 & 1.22 & 1.21 & 1.21 & 1.21 & 0.48 & 0.43 & 0.40 & 0.40 & 0.00 & 0.00 & -0.03 & -5.88 \\
\hline Vietnam & 0.04 & 0.03 & 0.03 & 0.03 & 1.61 & 1.61 & 1.60 & 1.60 & 0.06 & 0.05 & 0.05 & 0.05 & 0.00 & 0.00 & -0.01 & -9.43 \\
\hline Thailand & 0.03 & 0.03 & 0.03 & 0.03 & 1.63 & 1.63 & 1.63 & 1.63 & 0.05 & 0.05 & 0.05 & 0.05 & 0.00 & 0.00 & 0.00 & 0.00 \\
\hline Burma & 0.14 & 0.13 & 0.13 & 0.13 & 1.04 & 1.04 & 1.04 & 1.04 & 0.14 & 0.14 & 0.13 & 0.13 & 0.00 & 0.00 & -0.01 & -3.70 \\
\hline Serbia & 0.24 & 0.24 & 0.22 & 0.24 & 3.17 & 2.28 & 2.50 & 1.70 & 0.75 & 0.54 & 0.55 & 0.40 & -0.15 & -27.45 & -0.14 & -26.11 \\
\hline Mexico & 0.16 & 0.18 & 0.12 & 0.12 & 1.58 & 1.57 & 1.54 & 1.52 & 0.25 & 0.29 & 0.19 & 0.18 & -0.01 & -5.41 & -0.11 & -39.24 \\
\hline \multicolumn{17}{|l|}{ Africa } \\
\hline South Africa & 0.83 & 0.93 & 1.15 & 1.15 & 2.29 & 2.38 & 2.30 & 2.36 & 1.90 & 2.20 & 2.65 & 2.71 & 0.06 & 2.26 & 0.51 & 23.18 \\
\hline Nigeria & 1.10 & 1.20 & 1.20 & 1.20 & 1.01 & 0.93 & 1.04 & 1.04 & 1.11 & 1.12 & 1.25 & 1.25 & 0.00 & 0.00 & 0.13 & 11.91 \\
\hline Zambia & 0.16 & 0.30 & 0.38 & 0.38 & 1.81 & 1.35 & 1.27 & 1.27 & 0.30 & 0.41 & 0.48 & 0.48 & 0.00 & 0.00 & 0.06 & 15.57 \\
\hline Uganda & 0.05 & 0.05 & 0.05 & 0.05 & 0.60 & 0.60 & 0.60 & 0.60 & 0.03 & 0.03 & 0.03 & 0.03 & 0.00 & 0.00 & 0.00 & 0.00 \\
\hline Middle East & & & & & & & & & & & & & & & & \\
\hline Iran & 0.07 & 0.07 & 0.08 & 0.08 & 2.41 & 2.29 & 2.27 & 2.27 & 0.17 & 0.16 & 0.17 & 0.17 & 0.00 & 0.00 & 0.01 & 6.25 \\
\hline Turkey & 0.03 & 0.03 & 0.04 & 0.04 & 3.79 & 3.91 & 4.00 & 4.00 & 0.11 & 0.13 & 0.14 & 0.14 & 0.00 & 0.00 & 0.02 & 12.00 \\
\hline bthers & 0.49 & 0.46 & 0.50 & 0.51 & 2.06 & 2.03 & 1.99 & 2.00 & 1.01 & 0.94 & 1.00 & 1.02 & 0.02 & 2.21 & 0.08 & 8.41 \\
\hline
\end{tabular}

World and Selected Countries and Regions 
Table 12 Cottonseed Area, Yield, and Production

\begin{tabular}{|c|c|c|c|c|c|c|c|c|c|c|c|c|c|c|c|c|}
\hline \multirow{3}{*}{ Country / Region } & \multicolumn{4}{|c|}{$\begin{array}{c}\text { Area } \\
\text { (Million hectares) }\end{array}$} & \multicolumn{4}{|c|}{$\begin{array}{c}\text { Yield } \\
\text { (Metric tons per hectare) }\end{array}$} & \multicolumn{4}{|c|}{$\begin{array}{c}\text { Production } \\
\text { (Million metric tons) }\end{array}$} & \multicolumn{4}{|c|}{ Change in Production } \\
\hline & \multirow[b]{2}{*}{$2020 / 21$} & Prel. & \multicolumn{2}{|c|}{ 2022/23 Proj. } & \multirow[b]{2}{*}{$2020 / 21$} & \multirow{2}{*}{$\begin{array}{c}\text { Prel. } \\
2021 / 22\end{array}$} & \multicolumn{2}{|c|}{ 2022/23 Proj. } & \multirow[b]{2}{*}{$2020 / 21$} & \multirow{2}{*}{$\begin{array}{c}\text { Prel. } \\
2021 / 22\end{array}$} & \multicolumn{2}{|c|}{ 2022/23 Proj. } & \multicolumn{2}{|c|}{ From last month } & \multicolumn{2}{|c|}{ From last year } \\
\hline & & $2021 / 22$ & Mar & Apr & & & Mar & Apr & & & Mar & Apr & MMT & Percent & MMT & Percent \\
\hline United States & 3.33 & 4.16 & 3.01 & 3.01 & 1.22 & 1.16 & 1.34 & 1.34 & 4.05 & 4.83 & 4.04 & 4.04 & 0.00 & 0.00 & -0.79 & -16.30 \\
\hline Total Foreign & 27.49 & 27.87 & 28.05 & 28.08 & 1.34 & 1.33 & 1.35 & 1.36 & 36.97 & 36.99 & 37.89 & 38.23 & 0.34 & 0.89 & 1.24 & 3.35 \\
\hline \multicolumn{17}{|l|}{ South Asia } \\
\hline India & 13.29 & 12.37 & 12.70 & 12.70 & 0.88 & 0.84 & 0.82 & 0.82 & 11.72 & 10.36 & 10.40 & 10.40 & 0.00 & 0.00 & 0.04 & 0.41 \\
\hline Pakistan & 2.20 & 2.00 & 1.80 & 1.80 & 0.89 & 1.30 & 0.94 & 0.94 & 1.95 & 2.60 & 1.69 & 1.69 & 0.00 & 0.00 & -0.91 & -35.01 \\
\hline China & 3.20 & 3.10 & 3.05 & 3.05 & 3.63 & 3.39 & 3.79 & 3.92 & 11.60 & 10.50 & 11.56 & 11.95 & 0.39 & 3.39 & 1.45 & 13.81 \\
\hline \multicolumn{17}{|l|}{ Former Soviet Union - 12} \\
\hline Uzbekistan & 1.06 & 1.06 & 1.07 & 1.07 & 1.18 & 1.07 & 1.06 & 1.06 & 1.25 & 1.14 & 1.14 & 1.14 & 0.00 & 0.00 & 0.00 & 0.00 \\
\hline Turkmenistan & 0.55 & 0.55 & 0.55 & 0.55 & 0.66 & 0.64 & 0.64 & 0.64 & 0.36 & 0.35 & 0.35 & 0.35 & 0.00 & 0.00 & 0.00 & 0.00 \\
\hline Tajikistan & 0.16 & 0.17 & 0.17 & 0.17 & 1.11 & 1.09 & 1.11 & 1.11 & 0.18 & 0.19 & 0.19 & 0.19 & 0.00 & 0.00 & 0.00 & 1.08 \\
\hline Kazakhstan & 0.11 & 0.12 & 0.13 & 0.13 & 0.95 & 0.90 & 0.94 & 0.94 & 0.11 & 0.10 & 0.12 & 0.12 & 0.00 & 0.00 & 0.01 & 13.59 \\
\hline Argentina & 0.41 & 0.48 & 0.40 & 0.40 & 0.72 & 0.68 & 1.11 & 1.06 & 0.29 & 0.33 & 0.44 & 0.43 & -0.02 & -4.28 & 0.10 & 30.37 \\
\hline \multicolumn{17}{|l|}{ Middle East } \\
\hline Turkey & 0.35 & 0.45 & 0.56 & 0.56 & 2.71 & 2.76 & 2.88 & 2.88 & 0.95 & 1.24 & 1.60 & 1.60 & 0.00 & 0.00 & 0.36 & 28.93 \\
\hline Syria & 0.03 & 0.03 & 0.03 & 0.03 & 2.36 & 2.20 & 2.20 & 2.20 & 0.06 & 0.06 & 0.06 & 0.06 & 0.00 & 0.00 & 0.00 & 0.00 \\
\hline Iran & 0.10 & 0.10 & 0.10 & 0.10 & 1.35 & 1.35 & 1.35 & 1.35 & 0.13 & 0.14 & 0.14 & 0.14 & 0.00 & 0.00 & 0.00 & 0.00 \\
\hline Australia & 0.28 & 0.64 & 0.68 & 0.68 & 3.06 & 2.77 & 2.45 & 2.45 & 0.84 & 1.76 & 1.65 & 1.65 & 0.00 & 0.00 & -0.11 & -5.98 \\
\hline European Union & 0.34 & 0.32 & 0.31 & 0.31 & 1.59 & 1.66 & 1.62 & 1.70 & 0.54 & 0.53 & 0.50 & 0.53 & 0.02 & 4.58 & -0.01 & -1.69 \\
\hline \multicolumn{17}{|l|}{ Africa } \\
\hline Burkina Faso & 0.56 & 0.60 & 0.62 & 0.62 & 0.47 & 0.45 & 0.40 & 0.40 & 0.26 & 0.27 & 0.25 & 0.25 & 0.00 & 0.00 & -0.02 & -6.42 \\
\hline Mali & 0.17 & 0.72 & 0.69 & 0.69 & 0.51 & 0.56 & 0.41 & 0.41 & 0.08 & 0.40 & 0.28 & 0.28 & 0.00 & 0.00 & -0.12 & -29.95 \\
\hline Cameroon & 0.23 & 0.23 & 0.23 & 0.23 & 1.51 & 1.39 & 1.41 & 1.41 & 0.34 & 0.32 & 0.33 & 0.33 & 0.00 & 0.00 & 0.01 & 1.56 \\
\hline Cote d'Ivoire & 0.45 & 0.47 & 0.41 & 0.41 & 0.61 & 0.60 & 0.34 & 0.34 & 0.27 & 0.28 & 0.14 & 0.14 & 0.00 & 0.00 & -0.14 & -49.82 \\
\hline Benin & 0.62 & 0.64 & 0.58 & 0.58 & 0.78 & 0.73 & 0.75 & 0.75 & 0.48 & 0.47 & 0.43 & 0.43 & 0.00 & 0.00 & -0.04 & -8.32 \\
\hline Tanzania & 0.17 & 0.24 & 0.35 & 0.35 & 0.56 & 0.46 & 0.43 & 0.43 & 0.09 & 0.11 & 0.15 & 0.15 & 0.00 & 0.00 & 0.04 & 36.94 \\
\hline Egypt & 0.07 & 0.09 & 0.13 & 0.14 & 1.00 & 1.00 & 0.98 & 0.92 & 0.07 & 0.09 & 0.13 & 0.13 & 0.00 & 0.78 & 0.04 & 51.76 \\
\hline Nigeria & 0.27 & 0.27 & 0.27 & 0.27 & 0.56 & 0.56 & 0.56 & 0.56 & 0.15 & 0.15 & 0.15 & 0.15 & 0.00 & 0.00 & 0.00 & 0.00 \\
\hline Sudan & 0.20 & 0.20 & 0.20 & 0.20 & 1.41 & 1.41 & 1.53 & 1.53 & 0.28 & 0.28 & 0.31 & 0.31 & 0.00 & 0.00 & 0.02 & 8.51 \\
\hline Mexico & 0.15 & 0.15 & 0.20 & 0.20 & 2.40 & 2.71 & 2.39 & 2.39 & 0.35 & 0.42 & 0.48 & 0.48 & 0.00 & 0.00 & 0.06 & 14.63 \\
\hline Burma & 0.24 & 0.25 & 0.25 & 0.25 & 1.17 & 1.18 & 1.18 & 1.18 & 0.28 & 0.29 & 0.29 & 0.29 & 0.00 & 0.00 & 0.00 & 0.00 \\
\hline Dthers & 0.63 & 0.70 & 0.63 & 0.66 & 0.83 & 0.74 & 0.76 & 0.79 & 0.52 & 0.51 & 0.48 & 0.52 & 0.04 & 8.81 & 0.01 & 0.97 \\
\hline
\end{tabular}

World and Selected Countries and Regions 
Table 13 Peanut Area, Yield, and Production

\begin{tabular}{|c|c|c|c|c|c|c|c|c|c|c|c|c|c|c|c|c|}
\hline \multirow{3}{*}{ Country / Region } & \multicolumn{4}{|c|}{$\begin{array}{c}\text { Area } \\
\text { (Million hectares) }\end{array}$} & \multicolumn{4}{|c|}{$\begin{array}{c}\text { Yield } \\
\text { (Metric tons per hectare) }\end{array}$} & \multicolumn{4}{|c|}{$\begin{array}{c}\text { Production } \\
\text { (Million metric tons) }\end{array}$} & \multicolumn{4}{|c|}{ Change in Production } \\
\hline & \multirow[b]{2}{*}{$2020 / 21$} & \multirow{2}{*}{$\begin{array}{c}\text { Prel. } \\
\text { 2021/22 }\end{array}$} & \multicolumn{2}{|c|}{ 2022/23 Proj. } & \multirow[b]{2}{*}{$2020 / 21$} & \multirow{2}{*}{$\begin{array}{c}\text { Prel. } \\
2021 / 22\end{array}$} & \multicolumn{2}{|c|}{ 2022/23 Proj. } & \multirow[b]{2}{*}{$2020 / 21$} & \multirow{2}{*}{$\begin{array}{c}\text { Prel. } \\
2021 / 22\end{array}$} & \multicolumn{2}{|c|}{ 2022/23 Proj. } & \multicolumn{2}{|c|}{ From last month } & \multicolumn{2}{|c|}{ From last year } \\
\hline & & & Mar & Apr & & & Mar & Apr & & & Mar & Apr & MMT & Percent & MMT & Percent \\
\hline World & 29.93 & 31.05 & 29.63 & 29.33 & 1.68 & 1.62 & 1.69 & 1.70 & 50.26 & 50.44 & 50.11 & 49.76 & -0.35 & -0.69 & -0.68 & -1.35 \\
\hline United States & 0.65 & 0.62 & 0.56 & 0.56 & 4.27 & 4.63 & 4.50 & 4.50 & 2.79 & 2.89 & 2.53 & 2.53 & 0.00 & 0.00 & -0.36 & -12.44 \\
\hline Total Foreign & 29.27 & 30.43 & 29.07 & 28.77 & 1.62 & 1.56 & 1.64 & 1.64 & 47.46 & 47.56 & 47.58 & 47.24 & -0.35 & -0.73 & -0.32 & -0.67 \\
\hline China & 4.73 & 4.81 & 4.80 & 4.80 & 3.80 & 3.81 & 3.81 & 3.81 & 17.99 & 18.31 & 18.30 & 18.30 & 0.00 & 0.00 & -0.01 & -0.04 \\
\hline \multicolumn{17}{|l|}{ Africa } \\
\hline Nigeria & 3.25 & 3.50 & 3.40 & 3.40 & 1.30 & 1.21 & 1.32 & 1.32 & 4.23 & 4.23 & 4.50 & 4.50 & 0.00 & 0.00 & 0.27 & 6.43 \\
\hline Sudan & 3.20 & 3.94 & 3.00 & 3.00 & 0.87 & 0.60 & 0.83 & 0.83 & 2.77 & 2.36 & 2.50 & 2.50 & 0.00 & 0.00 & 0.15 & 6.16 \\
\hline Senegal & 1.23 & 1.21 & 1.20 & 1.20 & 1.47 & 1.38 & 1.42 & 1.42 & 1.80 & 1.68 & 1.70 & 1.70 & 0.00 & 0.00 & 0.02 & 1.31 \\
\hline Cameroon & 0.43 & 0.43 & 0.43 & 0.43 & 1.40 & 1.40 & 1.40 & 1.40 & 0.60 & 0.60 & 0.60 & 0.60 & 0.00 & 0.00 & 0.00 & 0.00 \\
\hline Ghana & 0.34 & 0.34 & 0.34 & 0.34 & 1.68 & 1.41 & 1.41 & 1.41 & 0.57 & 0.48 & 0.48 & 0.48 & 0.00 & 0.00 & 0.00 & 0.00 \\
\hline Chad & 0.76 & 0.80 & 0.80 & 0.80 & 1.11 & 1.13 & 1.13 & 1.13 & 0.84 & 0.90 & 0.90 & 0.90 & 0.00 & 0.00 & 0.00 & 0.00 \\
\hline Malawi & 0.40 & 0.40 & 0.40 & 0.40 & 0.88 & 0.88 & 0.88 & 0.88 & 0.35 & 0.35 & 0.35 & 0.35 & 0.00 & 0.00 & 0.00 & 0.00 \\
\hline Congo (Kinshasa) & 0.50 & 0.50 & 0.50 & 0.50 & 0.90 & 0.90 & 0.90 & 0.90 & 0.45 & 0.45 & 0.45 & 0.45 & 0.00 & 0.00 & 0.00 & 0.00 \\
\hline Niger & 0.90 & 0.92 & 0.92 & 0.92 & 0.66 & 0.56 & 0.60 & 0.60 & 0.59 & 0.52 & 0.55 & 0.55 & 0.00 & 0.00 & 0.03 & 5.97 \\
\hline Mali & 0.43 & 0.43 & 0.43 & 0.43 & 1.16 & 0.87 & 1.00 & 1.00 & 0.49 & 0.37 & 0.43 & 0.43 & 0.00 & 0.00 & 0.06 & 15.49 \\
\hline Uganda & 0.34 & 0.40 & 0.40 & 0.40 & 0.99 & 0.75 & 0.75 & 0.75 & 0.34 & 0.30 & 0.30 & 0.30 & 0.00 & 0.00 & 0.00 & 0.00 \\
\hline Burkina Faso & 0.65 & 0.60 & 0.60 & 0.60 & 0.97 & 0.80 & 0.83 & 0.83 & 0.63 & 0.48 & 0.50 & 0.50 & 0.00 & 0.00 & 0.02 & 4.82 \\
\hline Guinea & 0.98 & 1.10 & 1.10 & 1.10 & 1.10 & 1.21 & 1.09 & 1.09 & 1.07 & 1.34 & 1.20 & 1.20 & 0.00 & 0.00 & -0.14 & -10.11 \\
\hline Egypt & 0.06 & 0.06 & 0.06 & 0.06 & 3.20 & 3.20 & 3.20 & 3.20 & 0.21 & 0.21 & 0.21 & 0.21 & 0.00 & 0.00 & 0.00 & 0.00 \\
\hline Central African Republic & 0.10 & 0.10 & 0.10 & 0.10 & 1.50 & 1.50 & 1.50 & 1.50 & 0.15 & 0.15 & 0.15 & 0.15 & 0.00 & 0.00 & 0.00 & 0.00 \\
\hline South Africa & 0.04 & 0.04 & 0.03 & 0.03 & 2.21 & 1.48 & 2.33 & 2.33 & 0.09 & 0.07 & 0.07 & 0.07 & 0.00 & 0.00 & 0.01 & 7.69 \\
\hline Mozambique & 0.35 & 0.38 & 0.38 & 0.38 & 0.30 & 0.34 & 0.34 & 0.34 & 0.10 & 0.13 & 0.13 & 0.13 & 0.00 & 0.00 & 0.00 & 0.00 \\
\hline Cote d'Ivoire & 0.16 & 0.15 & 0.15 & 0.15 & 1.40 & 1.45 & 1.45 & 1.45 & 0.23 & 0.21 & 0.21 & 0.21 & 0.00 & 0.00 & 0.00 & 0.00 \\
\hline Benin & 0.17 & 0.19 & 0.19 & 0.19 & 1.01 & 0.92 & 0.92 & 0.92 & 0.18 & 0.18 & 0.18 & 0.18 & 0.00 & 0.00 & 0.00 & 0.00 \\
\hline \multicolumn{17}{|l|}{ South Asia } \\
\hline India & 6.02 & 5.71 & 5.50 & 5.20 & 1.11 & 1.19 & 1.21 & 1.21 & 6.70 & 6.80 & 6.65 & 6.30 & -0.35 & -5.26 & -0.50 & -7.35 \\
\hline Pakistan & 0.14 & 0.15 & 0.13 & 0.13 & 0.90 & 0.94 & 0.88 & 0.88 & 0.12 & 0.15 & 0.12 & 0.12 & 0.00 & 0.00 & -0.03 & -20.69 \\
\hline \multicolumn{17}{|l|}{ Southeast Asia } \\
\hline Indonesia & 0.55 & 0.55 & 0.54 & 0.54 & 1.78 & 1.76 & 1.76 & 1.76 & 0.97 & 0.96 & 0.95 & 0.95 & 0.00 & 0.00 & -0.01 & -1.04 \\
\hline Burma & 1.06 & 1.06 & 1.07 & 1.07 & 1.48 & 1.51 & 1.55 & 1.55 & 1.56 & 1.60 & 1.65 & 1.65 & 0.00 & 0.00 & 0.05 & 3.13 \\
\hline Vietnam & 0.17 & 0.16 & 0.16 & 0.16 & 2.50 & 2.50 & 2.50 & 2.50 & 0.41 & 0.40 & 0.39 & 0.39 & 0.00 & 0.00 & -0.01 & -3.00 \\
\hline Thailand & 0.02 & 0.02 & 0.02 & 0.02 & 1.67 & 1.67 & 1.67 & 1.67 & 0.04 & 0.04 & 0.04 & 0.04 & 0.00 & 0.00 & 0.00 & 0.00 \\
\hline \multicolumn{17}{|l|}{ South America } \\
\hline Argentina & 0.40 & 0.41 & 0.38 & 0.38 & 3.16 & 3.29 & 3.03 & 3.03 & 1.27 & 1.34 & 1.15 & 1.15 & 0.00 & 0.00 & -0.19 & -14.18 \\
\hline Brazil & 0.17 & 0.20 & 0.21 & 0.21 & 3.86 & 3.68 & 3.66 & 3.66 & 0.64 & 0.72 & 0.75 & 0.75 & 0.00 & 0.00 & 0.03 & 4.46 \\
\hline Mexico & 0.06 & 0.06 & 0.05 & 0.05 & 1.73 & 1.63 & 1.74 & 1.87 & 0.10 & 0.10 & 0.09 & 0.10 & 0.01 & 9.78 & 0.00 & 3.06 \\
\hline pthers & 1.70 & 1.83 & 1.79 & 1.79 & 1.16 & 1.19 & 1.18 & 1.17 & 1.97 & 2.17 & 2.10 & 2.10 & -0.01 & -0.33 & -0.08 & -3.45 \\
\hline
\end{tabular}

World and Selected Countries and Regions

Foreign Agricultural Service/USDA Global Market Analysis 
Table 14 Sunflowerseed Area, Yield, and Production

\begin{tabular}{|c|c|c|c|c|c|c|c|c|c|c|c|c|c|c|c|c|}
\hline \multirow{3}{*}{ Country / Region } & \multicolumn{4}{|c|}{$\begin{array}{c}\text { Area } \\
\text { (Million hectares) }\end{array}$} & \multicolumn{4}{|c|}{$\begin{array}{c}\text { Yield } \\
\text { (Metric tons per hectare) }\end{array}$} & \multicolumn{4}{|c|}{$\begin{array}{c}\text { Production } \\
\text { (Million metric tons) }\end{array}$} & \multicolumn{4}{|c|}{ Change in Production } \\
\hline & \multirow[b]{2}{*}{$2020 / 21$} & \multirow{2}{*}{$\begin{array}{c}\text { Prel. } \\
2021 / 22\end{array}$} & \multicolumn{2}{|c|}{ 2022/23 Proj. } & \multirow[b]{2}{*}{$2020 / 21$} & \multirow{2}{*}{$\begin{array}{c}\text { Prel. } \\
2021 / 22\end{array}$} & \multicolumn{2}{|c|}{ 2022/23 Proj. } & \multirow[b]{2}{*}{$2020 / 21$} & \multirow{2}{*}{$\begin{array}{c}\text { Prel. } \\
2021 / 22\end{array}$} & \multicolumn{2}{|c|}{ 2022/23 Proj. } & \multicolumn{2}{|c|}{ From last month } & \multicolumn{2}{|c|}{ From last year } \\
\hline & & & Mar & Apr & & & Mar & Apr & & & Mar & Apr & MMT & Percent & MMT & Percent \\
\hline World & 26.86 & 28.72 & 26.99 & 27.49 & 1.83 & 1.99 & 1.86 & 1.83 & 49.16 & 57.27 & 50.29 & 50.44 & 0.15 & 0.29 & -6.83 & -11.92 \\
\hline United States & 0.67 & 0.50 & 0.65 & 0.65 & 2.01 & 1.71 & 1.96 & 1.96 & 1.35 & 0.86 & 1.28 & 1.28 & 0.00 & 0.00 & 0.41 & 47.69 \\
\hline Total Foreign & 26.19 & 28.21 & 26.34 & 26.84 & 1.83 & 2.00 & 1.86 & 1.83 & 47.81 & 56.40 & 49.02 & 49.17 & 0.15 & 0.30 & -7.24 & -12.83 \\
\hline \multicolumn{17}{|l|}{ Former Soviet Union - 12} \\
\hline Russia & 8.35 & 9.61 & 9.00 & 9.11 & 1.59 & 1.62 & 1.78 & 1.78 & 13.27 & 15.57 & 16.00 & 16.25 & 0.25 & 1.59 & 0.68 & 4.38 \\
\hline Ukraine & 7.00 & 7.10 & 4.90 & 4.90 & 2.01 & 2.46 & 2.12 & 2.12 & 14.10 & 17.50 & 10.40 & 10.40 & 0.00 & 0.00 & -7.10 & -40.57 \\
\hline Kazakhstan & 0.75 & 0.94 & 1.09 & 1.09 & 1.13 & 1.10 & 1.20 & 1.20 & 0.84 & 1.03 & 1.30 & 1.30 & 0.00 & 0.00 & 0.27 & 26.36 \\
\hline European Union & 4.44 & 4.37 & 4.82 & 5.18 & 2.00 & 2.35 & 1.97 & 1.79 & 8.90 & 10.29 & 9.48 & 9.29 & -0.19 & -1.98 & -1.00 & -9.70 \\
\hline \multicolumn{17}{|l|}{ South America } \\
\hline Argentina & 1.67 & 1.96 & 2.20 & 2.20 & 2.05 & 2.07 & 1.73 & 1.73 & 3.43 & 4.05 & 3.80 & 3.80 & 0.00 & 0.00 & -0.25 & -6.17 \\
\hline Uruguay & 0.07 & 0.07 & 0.08 & 0.07 & 1.69 & 1.62 & 1.71 & 1.64 & 0.11 & 0.11 & 0.13 & 0.12 & -0.01 & -10.16 & 0.01 & 4.55 \\
\hline Bolivia & 0.14 & 0.14 & 0.14 & 0.14 & 1.39 & 1.39 & 1.40 & 1.40 & 0.19 & 0.20 & 0.20 & 0.20 & 0.00 & 0.00 & 0.00 & 1.54 \\
\hline China & 0.87 & 0.79 & 0.95 & 0.95 & 2.94 & 3.06 & 2.79 & 2.79 & 2.57 & 2.40 & 2.65 & 2.65 & 0.00 & 0.00 & 0.25 & 10.42 \\
\hline \multicolumn{17}{|l|}{ South Asia } \\
\hline India & 0.23 & 0.27 & 0.28 & 0.28 & 0.82 & 0.70 & 0.78 & 0.78 & 0.19 & 0.19 & 0.22 & 0.22 & 0.00 & 0.00 & 0.03 & 13.16 \\
\hline Pakistan & 0.06 & 0.10 & 0.10 & 0.11 & 1.47 & 1.38 & 1.35 & 1.35 & 0.09 & 0.14 & 0.14 & 0.15 & 0.01 & 9.63 & 0.01 & 4.96 \\
\hline \multicolumn{17}{|l|}{ Middle East } \\
\hline Turkey & 0.72 & 0.76 & 0.79 & 0.79 & 2.17 & 2.30 & 2.41 & 2.41 & 1.56 & 1.75 & 1.90 & 1.90 & 0.00 & 0.00 & 0.15 & 8.57 \\
\hline Iran & 0.04 & 0.04 & 0.04 & 0.04 & 1.08 & 1.00 & 1.02 & 1.02 & 0.04 & 0.04 & 0.04 & 0.04 & 0.00 & 0.00 & 0.00 & 0.00 \\
\hline Israel & 0.00 & 0.00 & 0.00 & 0.00 & 5.00 & 2.00 & 5.00 & 5.00 & 0.01 & 0.00 & 0.01 & 0.01 & 0.00 & 0.00 & 0.00 & 150.00 \\
\hline \multicolumn{17}{|l|}{ Africa } \\
\hline Egypt & 0.01 & 0.01 & 0.01 & 0.02 & 2.38 & 2.38 & 2.50 & 2.53 & 0.02 & 0.02 & 0.03 & 0.04 & 0.01 & 52.00 & 0.02 & 100.00 \\
\hline Morocco & 0.02 & 0.02 & 0.02 & 0.02 & 1.16 & 1.25 & 1.11 & 1.11 & 0.02 & 0.03 & 0.02 & 0.02 & 0.00 & 0.00 & -0.01 & -20.00 \\
\hline South Africa & 0.48 & 0.67 & 0.56 & 0.56 & 1.42 & 1.26 & 1.38 & 1.43 & 0.68 & 0.85 & 0.78 & 0.80 & 0.03 & 3.23 & -0.05 & -5.44 \\
\hline Burma & 0.60 & 0.60 & 0.60 & 0.60 & 0.65 & 0.65 & 0.65 & 0.65 & 0.39 & 0.39 & 0.39 & 0.39 & 0.00 & 0.00 & 0.00 & 0.00 \\
\hline Canada & 0.05 & 0.04 & 0.04 & 0.04 & 2.24 & 2.03 & 2.21 & 2.21 & 0.10 & 0.08 & 0.08 & 0.08 & 0.00 & 0.00 & 0.01 & 12.00 \\
\hline Australia & 0.02 & 0.02 & 0.03 & 0.03 & 1.44 & 1.58 & 1.48 & 1.48 & 0.03 & 0.03 & 0.04 & 0.04 & 0.00 & 0.00 & 0.01 & 23.33 \\
\hline bthers & 0.62 & 0.63 & 0.64 & 0.66 & 1.91 & 2.59 & 2.07 & 2.07 & 1.19 & 1.64 & 1.32 & 1.36 & 0.04 & 3.34 & -0.28 & -16.89 \\
\hline
\end{tabular}

World and Selected Countries and Regions 
Table 15 Rapeseed Area, Yield, and Production

\begin{tabular}{|c|c|c|c|c|c|c|c|c|c|c|c|c|c|c|c|c|}
\hline \multirow{3}{*}{ Country / Region } & \multicolumn{4}{|c|}{$\begin{array}{c}\text { Area } \\
\text { (Million hectares) }\end{array}$} & \multicolumn{4}{|c|}{$\begin{array}{c}\text { Yield } \\
\text { (Metric tons per hectare) }\end{array}$} & \multicolumn{4}{|c|}{$\begin{array}{c}\text { Production } \\
\text { (Million metric tons) }\end{array}$} & \multicolumn{4}{|c|}{ Change in Production } \\
\hline & \multirow[b]{2}{*}{$2020 / 21$} & Prel. & \multicolumn{2}{|c|}{ 2022/23 Proj. } & \multirow[b]{2}{*}{$2020 / 21$} & \multirow{2}{*}{$\begin{array}{c}\text { Prel. } \\
2021 / 22\end{array}$} & \multicolumn{2}{|c|}{ 2022/23 Proj. } & \multirow[b]{2}{*}{$2020 / 21$} & \multirow{2}{*}{$\begin{array}{c}\text { Prel. } \\
2021 / 22\end{array}$} & \multicolumn{2}{|c|}{ 2022/23 Proj. } & \multicolumn{2}{|c|}{ From last month } & \multicolumn{2}{|c|}{ From last year } \\
\hline & & $2021 / 22$ & Mar & Apr & & & Mar & Apr & & & Mar & Apr & MMT & Percent & MMT & Percent \\
\hline United States & 0.73 & 0.85 & 0.88 & 0.88 & 2.16 & 1.46 & 1.98 & 1.98 & 1.58 & 1.24 & 1.74 & 1.74 & 0.00 & 0.00 & 0.50 & 40.03 \\
\hline Total Foreign & 34.53 & 37.44 & 40.06 & 40.75 & 2.11 & 1.96 & 2.11 & 2.10 & 72.87 & 73.25 & 84.56 & 85.42 & 0.86 & 1.01 & 12.17 & 16.62 \\
\hline European Union & 5.32 & 5.39 & 5.92 & 5.94 & 3.14 & 3.23 & 3.30 & 3.29 & 16.73 & 17.39 & 19.50 & 19.54 & 0.04 & 0.18 & 2.15 & 12.35 \\
\hline United Kingdom & 0.38 & 0.31 & 0.36 & 0.36 & 2.73 & 3.20 & 3.40 & 3.40 & 1.04 & 0.98 & 1.23 & 1.23 & 0.00 & 0.00 & 0.24 & 24.87 \\
\hline Switzerland & 0.02 & 0.02 & 0.02 & 0.02 & 3.14 & 3.14 & 3.14 & 3.14 & 0.07 & 0.07 & 0.07 & 0.07 & 0.00 & 0.00 & 0.00 & 0.00 \\
\hline Canada & 8.33 & 8.95 & 8.60 & 8.60 & 2.34 & 1.54 & 2.21 & 2.21 & 19.49 & 13.75 & 19.00 & 19.00 & 0.00 & 0.00 & 5.25 & 38.16 \\
\hline China & 6.77 & 6.99 & 7.10 & 7.10 & 2.08 & 2.10 & 2.07 & 2.07 & 14.05 & 14.71 & 14.70 & 14.70 & 0.00 & 0.00 & -0.01 & -0.10 \\
\hline \multicolumn{17}{|l|}{ South Asia } \\
\hline India & 6.70 & 7.99 & 9.00 & 9.00 & 1.27 & 1.34 & 1.28 & 1.28 & 8.50 & 10.70 & 11.50 & 11.50 & 0.00 & 0.00 & 0.80 & 7.48 \\
\hline Pakistan & 0.25 & 0.33 & 0.35 & 0.39 & 1.35 & 1.49 & 1.40 & 1.00 & 0.34 & 0.49 & 0.49 & 0.39 & -0.10 & -20.41 & -0.10 & -20.41 \\
\hline Bangladesh & 0.59 & 0.61 & 0.25 & 0.81 & 1.33 & 1.35 & 0.92 & 1.30 & 0.79 & 0.82 & 0.23 & 1.05 & 0.82 & 356.52 & 0.23 & 27.74 \\
\hline Ukraine & 1.19 & 1.04 & 1.20 & 1.20 & 2.31 & 2.91 & 2.92 & 2.92 & 2.75 & 3.02 & 3.50 & 3.50 & 0.00 & 0.00 & 0.49 & 16.09 \\
\hline Belarus & 0.36 & 0.32 & 0.33 & 0.33 & 2.06 & 1.59 & 1.69 & 1.69 & 0.73 & 0.50 & 0.55 & 0.55 & 0.00 & 0.00 & 0.05 & 10.00 \\
\hline Kazakhstan & 0.12 & 0.12 & 0.13 & 0.13 & 1.23 & 1.24 & 1.48 & 1.48 & 0.15 & 0.15 & 0.19 & 0.19 & 0.00 & 0.00 & 0.04 & 29.45 \\
\hline Australia & 2.61 & 3.25 & 3.90 & 3.90 & 1.82 & 2.10 & 2.13 & 2.13 & 4.76 & 6.82 & 8.30 & 8.30 & 0.00 & 0.00 & 1.48 & 21.70 \\
\hline \multicolumn{17}{|l|}{ South America } \\
\hline Chile & 0.04 & 0.04 & 0.03 & 0.03 & 3.81 & 3.86 & 3.87 & 3.87 & 0.14 & 0.14 & 0.12 & 0.12 & 0.00 & 0.00 & -0.02 & -11.11 \\
\hline Paraguay & 0.05 & 0.04 & 0.05 & 0.05 & 1.41 & 1.45 & 1.51 & 1.51 & 0.07 & 0.06 & 0.07 & 0.07 & 0.00 & 0.00 & 0.00 & 6.25 \\
\hline Ethiopia & 0.03 & 0.03 & 0.03 & 0.03 & 1.80 & 1.80 & 1.80 & 1.80 & 0.05 & 0.05 & 0.05 & 0.05 & 0.00 & 0.00 & 0.00 & 0.00 \\
\hline pthers & 0.33 & 0.41 & 0.61 & 0.61 & 2.02 & 2.03 & 1.93 & 1.94 & 0.67 & 0.83 & 1.18 & 1.18 & 0.00 & 0.00 & 0.35 & 41.98 \\
\hline
\end{tabular}

World and Selected Countries and Regions 
Table 16 Copra, Palm Kernel, and Palm Oil Production

\begin{tabular}{|c|c|c|c|c|c|c|c|c|}
\hline \multirow{3}{*}{ Country / Region } & \multicolumn{4}{|c|}{$\begin{array}{c}\text { Production } \\
\text { (Million metric tons) } \\
\end{array}$} & \multicolumn{4}{|c|}{ Change in Production } \\
\hline & \multirow[b]{2}{*}{$2020 / 21$} & \multirow{2}{*}{$\begin{array}{c}\text { Prel. } \\
2021 / 22\end{array}$} & \multicolumn{2}{|c|}{ 2022/23 Proj. } & \multicolumn{2}{|c|}{ From last month } & \multicolumn{2}{|c|}{ From last year } \\
\hline & & & Mar & Apr & MMT & Percent & MMT & Percent \\
\hline \multicolumn{9}{|l|}{ Oilseed, Copra } \\
\hline Philippines & 2.40 & 2.60 & 2.60 & 2.60 & 0.00 & 0.00 & 0.00 & 0.00 \\
\hline Indonesia & 1.67 & 1.68 & 1.68 & 1.68 & 0.00 & 0.00 & 0.00 & 0.00 \\
\hline India & 0.93 & 0.94 & 0.94 & 0.94 & 0.00 & 0.00 & 0.00 & 0.00 \\
\hline Vietnam & 0.28 & 0.29 & 0.29 & 0.29 & 0.00 & 0.00 & 0.00 & 0.00 \\
\hline Papua New Guinea & 0.11 & 0.11 & 0.11 & 0.11 & 0.00 & 0.00 & 0.00 & 0.00 \\
\hline Sri Lanka & 0.07 & 0.11 & 0.07 & 0.07 & 0.00 & 0.00 & -0.03 & -30.00 \\
\hline Thailand & 0.07 & 0.09 & 0.09 & 0.09 & 0.00 & 0.00 & 0.00 & 0.00 \\
\hline Mexico & 0.04 & 0.05 & 0.05 & 0.05 & 0.00 & 0.00 & 0.00 & 0.00 \\
\hline Solomon Islands & 0.03 & 0.03 & 0.03 & 0.03 & 0.00 & 0.00 & 0.00 & 0.00 \\
\hline Cote d'Ivoire & 0.03 & 0.03 & 0.03 & 0.03 & 0.00 & 0.00 & 0.00 & 0.00 \\
\hline World & 5.78 & 6.07 & 6.03 & 6.03 & 0.00 & 0.00 & -0.04 & -0.66 \\
\hline \multicolumn{9}{|l|}{ Oilseed, Palm Kernel } \\
\hline Indonesia & 11.46 & 11.40 & 11.60 & 11.60 & 0.00 & 0.00 & 0.20 & 1.75 \\
\hline Malaysia & 4.34 & 4.45 & 4.90 & 4.90 & 0.00 & 0.00 & 0.45 & 10.11 \\
\hline Nigeria & 0.82 & 0.90 & 0.90 & 0.90 & 0.00 & 0.00 & 0.00 & 0.00 \\
\hline Thailand & 0.79 & 0.83 & 0.86 & 0.86 & 0.00 & 0.00 & 0.03 & 3.61 \\
\hline Colombia & 0.29 & 0.31 & 0.32 & 0.32 & 0.00 & 0.00 & 0.01 & 3.23 \\
\hline Guatemala & 0.17 & 0.18 & 0.18 & 0.18 & 0.00 & 0.00 & 0.00 & 0.00 \\
\hline Papua New Guinea & 0.16 & 0.17 & 0.17 & 0.17 & 0.00 & 0.00 & 0.01 & 6.25 \\
\hline Honduras & 0.13 & 0.16 & 0.16 & 0.16 & 0.00 & 0.00 & 0.00 & 0.00 \\
\hline Brazil & 0.14 & 0.15 & 0.15 & 0.15 & 0.00 & 0.00 & 0.01 & 7.14 \\
\hline Cameroon & 0.15 & 0.15 & 0.15 & 0.15 & 0.00 & 0.00 & 0.00 & 0.00 \\
\hline World & 19.09 & 19.33 & 20.08 & 20.08 & 0.00 & 0.00 & 0.75 & 3.88 \\
\hline \multicolumn{9}{|l|}{ Oil, Palm } \\
\hline Indonesia & 43.50 & 43.20 & 45.50 & 45.50 & 0.00 & 0.00 & 2.30 & 5.32 \\
\hline Malaysia & 17.85 & 18.15 & 19.20 & 19.20 & 0.00 & 0.00 & 1.05 & 5.79 \\
\hline Thailand & 2.96 & 3.38 & 3.26 & 3.26 & 0.00 & 0.00 & -0.12 & -3.55 \\
\hline Colombia & 1.56 & 1.75 & 1.77 & 1.77 & 0.00 & 0.00 & 0.02 & 1.14 \\
\hline Nigeria & 1.28 & 1.40 & 1.40 & 1.40 & 0.00 & 0.00 & 0.00 & 0.00 \\
\hline Guatemala & 0.87 & 0.88 & 0.91 & 0.91 & 0.00 & 0.00 & 0.03 & 3.41 \\
\hline Papua New Guinea & 0.60 & 0.63 & 0.65 & 0.65 & 0.00 & 0.00 & 0.03 & 4.84 \\
\hline Honduras & 0.45 & 0.60 & 0.60 & 0.60 & 0.00 & 0.00 & 0.00 & 0.00 \\
\hline Cote d'Ivoire & 0.55 & 0.58 & 0.60 & 0.60 & 0.00 & 0.00 & 0.03 & 5.26 \\
\hline Brazil & 0.55 & 0.55 & 0.57 & 0.57 & 0.00 & 0.00 & 0.02 & 3.64 \\
\hline World & 73.09 & 74.05 & 77.56 & 77.56 & 0.00 & 0.00 & 3.51 & 4.74 \\
\hline
\end{tabular}

World and Selected Countries and Regions 
Table 17 Cotton Area, Yield, and Production

\begin{tabular}{|c|c|c|c|c|c|c|c|c|c|c|c|c|c|c|c|c|}
\hline \multirow{3}{*}{ Country / Region } & \multicolumn{4}{|c|}{$\begin{array}{c}\text { Area } \\
\text { (Million hectares) }\end{array}$} & \multicolumn{4}{|c|}{$\begin{array}{c}\text { Yield } \\
\text { (Kilograms per hectare) }\end{array}$} & \multicolumn{4}{|c|}{$\begin{array}{c}\text { Production } \\
\text { (Million } 480 \text { lb. bales) }\end{array}$} & \multicolumn{4}{|c|}{ Change in Production } \\
\hline & \multirow[b]{2}{*}{$2020 / 21$} & Prel. & \multicolumn{2}{|c|}{ 2022/23 Proj. } & \multirow[b]{2}{*}{$2020 / 21$} & \multirow{2}{*}{$\begin{array}{c}\text { Prel. } \\
2021 / 22 \\
\end{array}$} & \multicolumn{2}{|c|}{ 2022/23 Proj. } & \multirow[b]{2}{*}{$2020 / 21$} & \multirow{2}{*}{$\begin{array}{c}\text { Prel. } \\
2021 / 22 \\
\end{array}$} & \multicolumn{2}{|c|}{ 2022/23 Proj. } & \multicolumn{2}{|c|}{ From last month } & \multicolumn{2}{|c|}{ From last year } \\
\hline & & $2021 / 22$ & Mar & Apr & & & Mar & Apr & & & Mar & Apr & MBales & Percent & MBales & Percent \\
\hline United States & 3.33 & 4.16 & 3.01 & 3.01 & 957 & 918 & 1,062 & 1,062 & 14.61 & 17.52 & 14.68 & 14.68 & 0.00 & 0.00 & -2.84 & -16.22 \\
\hline Total Foreign & 28.03 & 28.41 & 28.60 & 28.63 & 753 & 755 & 764 & 770 & 96.89 & 98.49 & 100.41 & 101.24 & 0.83 & 0.83 & 2.75 & 2.79 \\
\hline \multicolumn{17}{|l|}{ South Asia } \\
\hline India & 13.29 & 12.37 & 12.70 & 12.70 & 452 & 429 & 420 & 420 & 27.60 & 24.40 & 24.50 & 24.50 & 0.00 & 0.00 & 0.10 & 0.41 \\
\hline Pakistan & 2.20 & 2.00 & 1.80 & 1.80 & 445 & 653 & 472 & 472 & 4.50 & 6.00 & 3.90 & 3.90 & 0.00 & 0.00 & -2.10 & -35.00 \\
\hline China & 3.20 & 3.10 & 3.05 & 3.05 & 2,014 & 1,882 & 2,106 & 2,177 & 29.60 & 26.80 & 29.50 & 30.50 & 1.00 & 3.39 & 3.70 & 13.81 \\
\hline \multicolumn{17}{|l|}{ South America } \\
\hline Brazil & 1.37 & 1.60 & 1.63 & 1.63 & 1,720 & 1,595 & 1,777 & 1,736 & 10.82 & 11.72 & 13.30 & 13.00 & -0.30 & -2.26 & 1.28 & 10.92 \\
\hline Argentina & 0.41 & 0.48 & 0.40 & 0.40 & 717 & 680 & 653 & 626 & 1.35 & 1.50 & 1.20 & 1.15 & -0.05 & -4.17 & -0.35 & -23.33 \\
\hline Paraguay & 0.01 & 0.02 & 0.01 & 0.04 & 853 & 527 & 416 & 877 & 0.05 & 0.05 & 0.02 & 0.14 & 0.12 & 571.43 & 0.10 & 206.52 \\
\hline \multicolumn{17}{|l|}{ Africa } \\
\hline Cote d'Ivoire & 0.45 & 0.47 & 0.41 & 0.41 & 494 & 486 & 281 & 281 & 1.01 & 1.05 & 0.53 & 0.53 & 0.00 & 0.00 & -0.52 & -49.52 \\
\hline Benin & 0.62 & 0.64 & 0.58 & 0.58 & 513 & 483 & 492 & 492 & 1.45 & 1.42 & 1.30 & 1.30 & 0.00 & 0.00 & -0.12 & -8.45 \\
\hline Egypt & 0.07 & 0.09 & 0.13 & 0.14 & 720 & 717 & 703 & 661 & 0.22 & 0.28 & 0.42 & 0.43 & 0.01 & 1.19 & 0.15 & 51.79 \\
\hline Cameroon & 0.23 & 0.23 & 0.23 & 0.23 & 658 & 606 & 615 & 615 & 0.68 & 0.64 & 0.65 & 0.65 & 0.00 & 0.00 & 0.01 & 1.56 \\
\hline Tanzania & 0.17 & 0.24 & 0.35 & 0.35 & 284 & 231 & 218 & 218 & 0.22 & 0.26 & 0.35 & 0.35 & 0.00 & 0.00 & 0.10 & 37.25 \\
\hline Nigeria & 0.27 & 0.27 & 0.27 & 0.27 & 282 & 282 & 282 & 282 & 0.35 & 0.35 & 0.35 & 0.35 & 0.00 & 0.00 & 0.00 & 0.00 \\
\hline Zimbabwe & 0.24 & 0.25 & 0.24 & 0.24 & 222 & 191 & 195 & 195 & 0.25 & 0.22 & 0.22 & 0.22 & 0.00 & 0.00 & 0.00 & 0.00 \\
\hline Ethiopia & 0.08 & 0.08 & 0.08 & 0.08 & 637 & 637 & 637 & 637 & 0.24 & 0.24 & 0.24 & 0.24 & 0.00 & 0.00 & 0.00 & 0.00 \\
\hline \multicolumn{17}{|l|}{ Former Soviet Union - 12} \\
\hline Uzbekistan & 1.06 & 1.06 & 1.07 & 1.07 & 653 & 596 & 590 & 590 & 3.18 & 2.90 & 2.90 & 2.90 & 0.00 & 0.00 & 0.00 & 0.00 \\
\hline Turkmenistan & 0.55 & 0.55 & 0.55 & 0.55 & 368 & 356 & 356 & 356 & 0.92 & 0.90 & 0.90 & 0.90 & 0.00 & 0.00 & 0.00 & 0.00 \\
\hline Tajikistan & 0.16 & 0.17 & 0.17 & 0.17 & 619 & 608 & 615 & 615 & 0.46 & 0.48 & 0.48 & 0.48 & 0.00 & 0.00 & 0.01 & 1.05 \\
\hline Kazakhstan & 0.11 & 0.12 & 0.13 & 0.13 & 534 & 502 & 523 & 523 & 0.27 & 0.27 & 0.30 & 0.30 & 0.00 & 0.00 & 0.04 & 13.21 \\
\hline \multicolumn{17}{|l|}{ Middle East } \\
\hline Turkey & 0.35 & 0.45 & 0.56 & 0.56 & 1,804 & 1,839 & 1,922 & 1,922 & 2.90 & 3.80 & 4.90 & 4.90 & 0.00 & 0.00 & 1.10 & 28.95 \\
\hline Syria & 0.03 & 0.03 & 0.03 & 0.03 & 1,219 & 1,132 & 1,132 & 1,132 & 0.14 & 0.13 & 0.13 & 0.13 & 0.00 & 0.00 & 0.00 & 0.00 \\
\hline Mexico & 0.15 & 0.15 & 0.20 & 0.20 & 1,532 & 1,725 & 1,524 & 1,524 & 1.02 & 1.22 & 1.40 & 1.40 & 0.00 & 0.00 & 0.18 & 14.75 \\
\hline Burma & 0.24 & 0.25 & 0.25 & 0.25 & 635 & 644 & 644 & 644 & 0.70 & 0.73 & 0.73 & 0.73 & 0.00 & 0.00 & 0.00 & 0.00 \\
\hline pthers & 1.71 & 1.76 & 1.70 & 1.69 & 577 & 559 & 564 & 574 & 4.54 & 4.52 & 4.40 & 4.45 & 0.05 & 1.23 & -0.07 & -1.48 \\
\hline
\end{tabular}

World and Selected Countries and Regions 


\section{TABLE 18}

The table below presents a record of the April projection and the final Estimate. Using world wheat production as an example, the "root mean square error" means that chances are 2 out of 3 that the current forecast will not be above or below the final estimate by more than 0.5 percent. Chances are 9 out of $10(90 \%$ confidence level) that the difference will not exceed 0.8 percent. The average difference between the April projection and the final estimate is 2.0 million tons, ranging from 0.1 million to 6.8 million tons. The April projection has been below the estimate 27 times and above 14 times.

RELIABILITY OF PRODUCTION PROJECTIONS 1/

\begin{tabular}{|c|c|c|c|c|c|c|c|}
\hline \multirow{3}{*}{$\begin{array}{c}\text { COMMODITY AND } \\
\text { REGION }\end{array}$} & \multirow{3}{*}{$\begin{array}{l}\text { Root mean } \\
\text { square error }\end{array}$} & \multirow{3}{*}{$\begin{array}{c}90 \text { percent } \\
\text { confidence } \\
\text { interval }\end{array}$} & \multicolumn{5}{|c|}{ Difference between forecast and final estimate } \\
\hline & & & \multirow[b]{2}{*}{ Average } & \multirow[b]{2}{*}{ Smallest } & \multirow[b]{2}{*}{ Largest } & \multicolumn{2}{|c|}{ Years } \\
\hline & & & & & & $\begin{array}{c}\text { Below } \\
\text { final }\end{array}$ & $\begin{array}{l}\text { Above } \\
\text { final }\end{array}$ \\
\hline & & & & ion metric & & & \\
\hline \multicolumn{8}{|l|}{ WHEAT } \\
\hline World & 0.5 & 0.8 & 2.0 & 0.1 & 6.8 & 27 & 14 \\
\hline U.S. & 0.2 & 0.3 & 0.1 & 0.0 & 0.3 & 19 & 11 \\
\hline Foreign & 0.5 & 0.9 & 2.0 & 0.2 & 6.8 & 27 & 14 \\
\hline \multicolumn{8}{|l|}{ COARSE GRAINS 2/ } \\
\hline World & 1.1 & 1.8 & 9.1 & 0.3 & 42.1 & 33 & 8 \\
\hline U.S. & 0.2 & 0.3 & 0.2 & 0.0 & 1.8 & 17 & 10 \\
\hline Foreign & 1.5 & 2.5 & 9.2 & 0.3 & 42.1 & 33 & 8 \\
\hline \multicolumn{8}{|l|}{ RICE (Milled) } \\
\hline World & 1.3 & 2.1 & 3.3 & 0.0 & 13.3 & 33 & 8 \\
\hline U.S. & 1.3 & 2.2 & 0.0 & 0.0 & 0.2 & 11 & 5 \\
\hline Foreign & 1.3 & 2.1 & 3.3 & 0.0 & 13.3 & 33 & 8 \\
\hline \multicolumn{8}{|l|}{ SOYBEANS } \\
\hline World & 1.6 & 2.7 & 2.6 & 0.2 & 7.9 & 24 & 17 \\
\hline U.S. & 1.4 & 2.3 & 0.7 & 0.0 & 3.2 & 18 & 16 \\
\hline Foreign & 2.7 & 4.5 & 2.3 & 0.0 & 8.1 & 26 & 15 \\
\hline COTTON & \multicolumn{7}{|c|}{---Million 480-lb. bales--- } \\
\hline World & 1.7 & 2.8 & 1.2 & 0.0 & 5.2 & 29 & 11 \\
\hline U.S. & 0.3 & 0.5 & 0.0 & 0.0 & 0.2 & 17 & 16 \\
\hline Foreign & 2.0 & 3.3 & 1.2 & 0.0 & 5.1 & 28 & 12 \\
\hline UNITED STATES & \multicolumn{7}{|c|}{------Million bushels------ } \\
\hline CORN & 0.2 & 0.3 & 6 & 0 & 72 & 1 & 5 \\
\hline SORGHUM & 0.1 & 0.2 & 0 & 0 & 4 & 0 & 2 \\
\hline BARLEY & 1.0 & 1.6 & 1 & 0 & 11 & 20 & 4 \\
\hline OATS & 0.4 & 0.7 & 0 & 0 & 2 & 5 & 4 \\
\hline
\end{tabular}

1/ Marketing years 1981/82 through 2021/22 for grains, soybeans, and cotton. Final for grains, soybeans, and cotton is defined as the first November estimate following the marketing year for 1981/82 through 2020/21, and for 2021/22 the last month's estimate.

2/ Includes corn, sorghum, barley, oats, rye, millet, and mixed grain

April 2023

Global Market Analysis, FAS, USDA 